TRANSACTIONS OF THE

AMERICAN MATHEMATICAL SOCIETY

Volume 358, Number 3, Pages 1117-1163

S 0002-9947(05)03728-1

Article electronically published on April 22, 2005

\title{
DUALITY FOR HOPF ORDERS
}

\author{
ROBERT G. UNDERWOOD AND LINDSAY N. CHILDS
}

\begin{abstract}
In this paper we use duality to construct new classes of Hopf orders in the group algebra $K C_{p^{3}}$, where $K$ is a finite extension of $\mathbb{Q}_{p}$ and $C_{p^{3}}$ denotes the cyclic group of order $p^{3}$. Included in this collection is a subcollection of Hopf orders which are realizable as Galois groups.
\end{abstract}

\section{INTRODUCTION}

Let $p$ be a prime number and let the field $K$ be a finite extension of $\mathbb{Q}_{p}$. Let $\operatorname{ord}(a)$ be the valuation of $a$ in $K$, normalized so that $\operatorname{ord}(\pi)=1$, where $\pi$ is a parameter for $K$, and let $R$ be the valuation ring of $K$. Let $C_{p^{n}}$ denote the cyclic group of order $p^{n}$.

The classification of Hopf orders in $K C_{p^{n}}$ is a problem that has been under investigation since the 1970's. Complete classifications are known only for the cases $n=1,2$; see [TO70], La76], Gr92], By93, Un94, [C00] and section 1, below. For $n=3$ the first author constructed a class of Hopf orders by cohomological methods extending Greither's for $n=2$ in Gr92. These Hopf orders are extensions of rank $p$ Larson orders by rank $p^{2}$ Hopf orders that are duals of Larson orders, which we call cohomological Hopf orders. In CU03] we constructed Hopf orders in $K C_{p^{n}}$ for all $n$ using isogenies of polynomial formal groups, orders that we will call formal group Hopf orders. We showed that for $n=3$ there exist formal group Hopf orders that are not cohomological.

Classifying Hopf orders in $K C_{p^{n}}$ remains open for $n \geq 3$.

This paper uses duality to construct new Hopf orders in $K C_{p^{3}}$.

The paper contains five sections.

In section 1, we review the structure of $R$-Hopf orders in $K C_{p}$ and $K C_{p^{2}}$. Assuming $K$ contains $\zeta_{2}$, a primitive $p^{2}$ nd root of unity, we give a new duality construction of all Hopf orders in $K C_{p^{2}}$. We then define "triangular" Hopf orders in $K C_{p^{3}}$, recall and re-parametrize as triangular Hopf orders the cohomological Hopf orders in $K C_{p^{3}}$ from [Un96], and introduce ILD Hopf orders in $K C_{p^{3}}$, a collection of triangular Hopf orders induced from cohomological Hopf orders by base change from the dual Larson orders defining the cohomological Hopf orders.

Section 2 is devoted to duality results needed later in the paper. Included is a precise determination of the valuation of $G(x, y)-1$, where $G(x, y)$ is the Gauss sum defined in GC98. As a consequence of these duality results, we show that

Received by the editors July 18, 2003 and, in revised form, April 16, 2004.

2000 Mathematics Subject Classification. Primary 13C05, 13E15, 16W30; Secondary 14L05, $12 \mathrm{~F} 10$.

(C)2005 American Mathematical Society

Reverts to public domain 28 years from publication 
most triangular Hopf orders are induced Hopf orders, that is, are "induced from both ends" from a cohomological Hopf order (Theorem 2.8).

Section 3 contains the generalization to $K C_{p^{3}}$ of the duality construction of Section 1. The triangular Hopf orders obtained are called duality Hopf orders. This collection is distinct from the collection of ILD orders.

In section 4, we recall the class of formal group Hopf orders ([CU03). For $n=3$ we find inequalities on the parameters sufficient for the existence of formal group Hopf orders, independent of and sharper than the main result of CU03, and recall from CU03 that under suitable conditions on the parameters, a formal group Hopf order is not a triangular Hopf order. We find that the dual of any formal group Hopf order is a triangular Hopf order that is never itself a formal group Hopf order. We find conditions for a formal group Hopf order itself to be triangular.

Finally, in section 5, we show that almost none of the Hopf orders of rank $p^{3}$ defined in this paper are realizable. By a theorem of N. Byott By04, a Hopf order $H$ with local dual $H^{*}$ is realizable if and only if $H^{*}$ is monogenic as an $R$-algebra. For known realizable Hopf orders of rank $p^{2}$ and $p^{3}$ we find algebra generators for their duals, and we find a new class of realizable Hopf orders of rank $p^{3}$. This new class includes triangular Hopf orders that are not among any of the families constructed in sections 1,3 and 4 . We conjecture that we have not constructed all realizable Hopf orders of rank $p^{3}$, and so the problem of constructing all Hopf orders of rank $p^{n}, n \geq 3$, remains open.

The first author thanks the University at Albany for its hospitality during this research; the second author thanks Auburn University Montgomery and Union College for their hospitality during this research. Both authors thank the referee for a thorough reading of this paper; the resulting comments and suggestions improved the content and presentation of this work.

\section{KNOWN Hopf ORders OF RANK $p, p^{2}, p^{3}$}

Assume $K$ is a field containing $\mathbb{Q}_{p}$ and a primitive $p$ th root of unity $\zeta_{1}$. Let $\operatorname{ord}\left(\zeta_{1}-1\right)=e^{\prime}, \operatorname{ord}(p)=e=(p-1) e^{\prime}$. For an integer $i$ with $0 \leq i \leq e^{\prime}$, set $i^{\prime}=e^{\prime}-i$.

Orders in $K C_{p}$. Orders in $K C_{p}$ were classified by J. Tate and F. Oort TO70, (cf. [C00, Chapters 4 and 5]). It is convenient to describe them as follows: any $R$-Hopf order in $K C_{p}$ is of the form

$$
H(i)=R\left[\frac{g-1}{\pi^{i}}\right],
$$

$\langle g\rangle=C_{p}$, for some integer $i, 0 \leq i \leq e^{\prime}$. Such $R$-Hopf orders are called Larson orders in $K C_{p}$ La76, Un94.

Assuming $K$ contains a primitive $p$ th root of unity $\zeta_{1}$, the character group $\hat{C}_{p}$ of $C_{p}$ is isomorphic to $C_{p}$, and is generated by the character $\gamma$ with $\gamma^{j}\left(g^{k}\right)=\zeta_{1}^{j k}$ for $j, k=0, \ldots, p-1$. The linear dual of $H(i)$ is then the Larson order

$$
H\left(i^{\prime}\right)=R\left[\frac{\gamma-1}{\pi^{i^{\prime}}}\right]
$$

cf. [Gr92, Lemma 3.1] or [C00, 21.2]. 
Orders in $K C_{p^{2}}$. Hopf orders in $K C_{p^{2}}$ were classified by Byott By93, Greither Gr92 and Underwood [Un94, assuming that $K$ contains a primitive $p$ th root of unity $\zeta_{1}$. Let $C_{p^{2}}=\langle g\rangle$ and let

$$
e_{m}=\frac{1}{p} \sum_{k=0}^{p-1} \zeta_{1}^{-m k} g^{p k}, 0 \leq m \leq p-1,
$$

denote the minimal idempotents of $K\left\langle g^{p}\right\rangle$. Greither's classification of Hopf orders in $K C_{p^{2}}$ uses elements of $K\left\langle g^{p}\right\rangle$ defined as follows: define

$$
a: K^{*} \rightarrow K\left\langle g^{p}\right\rangle^{*}
$$

by sending $v$ in $K^{*}$ to $a_{v}=\sum_{m=0}^{p-1} v^{m} e_{m}$. Then $a$ is a multiplicative homomorphism, with $a_{1}=1$ and $a_{\zeta_{1}}=g^{p}$. Greither showed that for $v \in R$,

$$
a_{v}-1 \text { is in } \pi^{\ell} H(i) \text { iff } \operatorname{ord}(v-1) \geq i^{\prime}+\ell .
$$

Using a cohomological argument, Greither showed that given $i, j$ with $0 \leq i, j \leq e^{\prime}$ satisfying the "p-adic" condition $p j \leq i$, the order

$$
H(i, j, v)=R\left[\frac{g^{p}-1}{\pi^{i}}, \frac{a_{v} g-1}{\pi^{j}}\right]=H(i)\left[\frac{a_{v} g-1}{\pi^{j}}\right]
$$

is a Hopf order in $K C_{p^{2}}$ provided that

$$
\operatorname{ord}(v-1) \geq \max \left\{\left\lceil i^{\prime}+j / p\right\rceil,\left\lceil i^{\prime} / p+j\right\rceil\right\},
$$

where $\lceil x\rceil$ denotes the smallest positive integer $\geq x$. The map sending $g$ to $\bar{g}(=$ the coset of $g$ modulo $\left\langle g^{p}\right\rangle$ ) induces a short exact sequence of Hopf algebras,

$$
K \rightarrow K\left\langle g^{p}\right\rangle \rightarrow K C_{p^{2}} \rightarrow K\langle\bar{g}\rangle \rightarrow K
$$

and a sequence of Hopf orders,

$$
R \rightarrow H(i) \rightarrow H(i, j, v) \rightarrow H(j) \rightarrow R .
$$

Thus Greither's orders are naturally presented as extensions of rank $p$ Larson orders, and in fact that is how they were constructed.

If $v=1$, or more generally, if $\operatorname{ord}(v-1) \geq i^{\prime}+j$, then $H(i, j, v)$ is isomorphic to the Larson order $H(i, j, 1):=H(i, j)$ (which is only defined if the p-adic condition $p j \leq i$ holds). Generally, we have from Gr92, (cf. [C00, (31.12)]),

$$
H(i, j, v) \equiv H(i, j, w) \text { iff } \operatorname{ord}(v-w) \geq i^{\prime}+j .
$$

Suppose $K$ contains a primitive $p^{2}$ nd root of unity $\zeta_{2}$ with $\zeta_{1}=\zeta_{2}^{p}$. Let $\hat{C}_{p^{2}}=\langle\gamma\rangle$ be the character group of $C_{p^{2}}$ and let

$$
\hat{e}_{m}=\frac{1}{p} \sum_{k=0}^{p-1} \zeta_{1}^{-m k} \gamma^{p k}, 0 \leq m \leq p-1,
$$

denote the minimal idempotents of $K\left\langle\gamma^{p}\right\rangle$. Then the linear dual of $H(i, j, v)$ is the Hopf order $H\left(j^{\prime}, i^{\prime}, \hat{v}\right)$ in $K\langle\gamma\rangle$, where $\hat{v}=\left(v \zeta_{2}\right)^{-1}$, as will be verified below.

Underwood [Un94] showed that every Hopf order in $K C_{p^{2}}$ is either a Greither order or the dual of a Greither order.

As a model for a construction in section 3, we now give a new construction of Hopf orders in $K C_{p^{2}}$. Our approach is based on the following result, C00, (31.2)], which is a slight generalization of [GC98, Lemma 2.1]. 
Proposition 1.1. Let $G$ be a finite p-group, and let $G^{\prime}$ be a subgroup of index $p$, with $G=\left\langle G^{\prime}, g\right\rangle$. Let $A$ be a Hopf order in $K G^{\prime}$. Let $u$ be a non-zero element of $K G^{\prime}$ and $0 \leq k \leq e^{\prime}$. Then

$$
H=A[y], y=\frac{u g-1}{\pi^{k}},
$$

is an order in $K G$, free over $A$ with basis $\left\{1, y, \ldots, y^{p-1}\right\}$, iff

$$
g^{p} u^{p} \equiv 1 \quad\left(\bmod \pi^{p k} A\right),
$$

and is a Hopf order in $K G$ if in addition, $u$ is a unit of $A$ and

$$
\Delta(u) \equiv u \otimes u \quad\left(\bmod \pi^{k}(A \otimes A)\right) .
$$

In Proposition 1.1 the algebra condition $g^{p} u^{p} \equiv 1\left(\bmod \pi^{p k} A\right)$ is generally easier to understand than the coalgebra condition $\Delta(u) \equiv u \otimes u\left(\bmod \pi^{k}(A \otimes A)\right)$.

In Section 31 of [C00, Hopf orders in $K C_{p^{2}}$ were constructed using Proposition 1.1. applying both the algebra and coalgebra conditions. However, if we assume $K$ contains $\zeta_{2}$, we can construct the same Hopf orders using only the algebra condition and duality. Denote $\left(\zeta_{2} v\right)^{-1}=\hat{v}$. Then $\operatorname{ord}\left(\zeta_{2} v-1\right)=\operatorname{ord}(\hat{v}-1)$.

Theorem 1.2. Assume $K$ contains $\zeta_{2}$, a primitive $p^{2} n d$ root of unity, let $0 \leq i, j \leq$ $e^{\prime}$, and let $v$ be a unit in $R$. Let

$$
A(i, j, v)=R\left[\frac{g^{p}-1}{\pi^{i}}, \frac{a_{v} g-1}{\pi^{j}}\right]
$$

and assume that $v$ satisfies the algebra condition

$$
\operatorname{ord}\left(\zeta_{1} v^{p}-1\right) \geq i^{\prime}+p j \text {. }
$$

Let

$$
A\left(j^{\prime}, i^{\prime}, \hat{v}\right)=R\left[\frac{\gamma^{p}-1}{\pi^{j^{\prime}}}, \frac{a_{\hat{v}} \gamma-1}{\pi^{i^{\prime}}}\right]
$$

with $\langle\gamma\rangle=\hat{C}_{p^{2}}$, and assume that $\hat{v}$ satisfies the algebra condition

$$
\operatorname{ord}\left(v^{p}-1\right) \geq j+p i^{\prime}
$$

Then $A(i, j, v)$ is an $R$-Hopf order in $K C_{p^{2}}$.

Proof. First observe that $\left(a_{v} g\right)^{p}=a_{v^{p}} a_{\zeta_{1}}=a_{v^{p} \zeta_{1}}$, and so by (1),

$$
\left(a_{v} g\right)^{p} \equiv 1 \quad\left(\bmod \pi^{p j} H(i)\right) \quad \text { iff } \quad v^{p} \zeta_{1} \equiv 1 \quad\left(\bmod \pi^{i^{\prime}+p j} R\right) .
$$

Thus Proposition 1.1 implies that $A(i, j, v)$ is an algebra that is free of rank $p$ over $H(i)$. Similarly for $A\left(j^{\prime}, i^{\prime}, \hat{v}\right)$. Now let $A(i, j, v)^{*}$ denote the linear dual of $A(i, j, v)$. Let $\operatorname{tr}: K C_{p^{2}} \rightarrow K$ be the trace map defined by $\operatorname{tr}(x)=\sum_{i=0}^{p^{2}-1} \sigma_{i}(x)$, where $\sigma_{i}: K C_{p^{2}} \rightarrow K$ is given by $\sigma_{i}(g)=\zeta_{2}^{i}$. Let $\left\{\alpha_{j}\right\}$ be an $R$-basis for $A(i, j, v)$. Then there exists a collection $\left\{\beta_{j}\right\} \subseteq K C_{p^{2}}$ for which $\operatorname{tr}\left(\alpha_{i} \beta_{j}\right)=\delta_{i j}$, where $\delta_{i j}$ is Kronecker's symbol. One has that

$$
A(i, j, v)^{*}=\left\{x \in K C_{p^{2}} \mid \operatorname{tr}(x A(i, j, v)) \subseteq R\right\},
$$

and $A(i, j, v)^{*}$ is a free $R$-module with basis $\left\{\beta_{j}\right\}$.

If we show that $A\left(j^{\prime}, i^{\prime}, \hat{v}\right)=A(i, j, v)^{*}$, then $A(i, j, v)^{*}$ will be closed under the multiplication map induced by the multiplication on $K \hat{C}_{p^{2}}$, which means that $A(i, j, v)$ will be closed under the comultiplication induced by that on $K C_{p^{2}}$, and hence $A(i, j, v)$ will be an $R$-order and an $R$-coalgebra. It will then follow that 
$A(i, j, v)$ is closed under the antipode, and consequently, $A(i, j, v)$ will be an $R$ Hopf order in $K C_{p^{2}}$.

To show that $A\left(j^{\prime}, i^{\prime}, \hat{v}\right)=A(i, j, v)^{*}$ we first show that their discriminants are equal.

By the method of [Un94, Theorem 2.0, Part 2], one has

$$
\operatorname{disc}(A(i, j, v))=\pi^{p^{2}(p-1)\left(i^{\prime}+j^{\prime}\right)} R
$$

and

$$
\operatorname{disc}\left(A\left(j^{\prime}, i^{\prime}, \hat{v}\right)\right)=\pi^{p^{2}(p-1)(i+j)} R .
$$

(Note: this shows that the discriminant of $A(i, j, v)$ depends only on $i$ and $j$.)

Let $M$ be the matrix which multiplies the basis

$$
\left\{\left(\frac{g^{p}-1}{\pi^{i}}\right)^{a}\left(\frac{a_{v} g-1}{\pi^{j}}\right)^{b}\right\}
$$

of $A(i, j, v)$ to give a basis of $R C_{p^{2}}$. Then

$$
\operatorname{disc}\left(R C_{p^{2}}\right)=\operatorname{det}^{2}(M) \operatorname{disc}(A(i, j, v)) .
$$

Moreover, $M^{T}$ is the matrix which multiplies a basis of the maximal integral order $\left(R C_{p^{2}}\right)^{*}=R^{p^{2}}$ to give a basis for $A(i, j, v)^{*}$, hence

$$
\begin{aligned}
\operatorname{disc}\left(A(i, j, v)^{*}\right) & =\operatorname{det}^{2}\left(M^{T}\right) \operatorname{disc}\left(R^{p^{2}}\right) \\
& =\operatorname{det}^{2}(M) \operatorname{disc}\left(R^{p^{2}}\right) .
\end{aligned}
$$

Now by a well-known formula, $\operatorname{disc}\left(R C_{p^{2}}\right) \operatorname{disc}\left(R^{p^{2}}\right)=\pi^{2 p^{2} e} R$, thus

$$
\operatorname{disc}(A(i, j, v)) \operatorname{disc}\left(A(i, j, v)^{*}\right)=\pi^{2 p^{2} e} R,
$$

whence $\operatorname{disc}\left(A(i, j, v)^{*}\right)=\operatorname{disc}\left(A\left(j^{\prime}, i^{\prime}, \hat{v}\right)\right)$.

We next show that

$$
\left\langle A(i, j, v), A\left(j^{\prime}, i^{\prime}, \hat{v}\right)\right\rangle \subseteq R
$$

that is,

$$
\left\langle\left(g^{p}-1\right)^{q}\left(a_{v} g-1\right)^{r},\left(\gamma^{p}-1\right)^{s}\left(a_{\hat{v}} \gamma-1\right)^{t}\right\rangle \in \pi^{q i+r j+s j^{\prime}+t i^{\prime}} R
$$

for $q, r, s, t=0, \ldots, p-1$. Here $\langle\rangle:, K C_{p^{2}} \times K \hat{C}_{p^{2}} \mapsto K$ is the duality map.

We need the following lemma, whose proof is a routine computation left to the reader.

Lemma 1.3. Let $e_{i}$ denote the minimal idempotents of $K C_{p}$. Then

$$
\left\langle e_{j} g^{p a+b}, e_{k} \gamma^{p c+d}\right\rangle=\zeta_{2}^{(p a+b)(p c+d)}
$$

if $j=d, k=b$, and is 0 otherwise. 
Let $S=\left\langle\left(g^{p}-1\right)^{q}\left(a_{v} g-1\right)^{r},\left(\gamma^{p}-1\right)^{s}\left(a_{\hat{v}} \gamma-1\right)^{t}\right\rangle$, and let $\sum_{c, d, e, f=0}^{q, r, s, t}$ denote the $\operatorname{sum} \sum_{c=0}^{q} \sum_{d=0}^{r} \sum_{e=0}^{s} \sum_{f=0}^{t}$. Then

$$
\begin{aligned}
S & =\sum_{c, d, e, f=0}^{q, r, s, t} C(c, d, e, f)\left\langle g^{p c}\left(a_{v} g\right)^{d}, \gamma^{p e}\left(a_{\hat{v}}\right)^{f}\right\rangle \\
& =\sum_{c, d, e, f=0}^{q, r, s, t} C(c, d, e, f)\left\langle a_{v^{d}} g^{p c+d}, a_{\hat{v} f} \gamma^{p e+f}\right\rangle \\
& =\sum_{c, d, e, f=0}^{q, r, s, t} C(c, d, e, f) \sum_{i, j} v^{d i} \hat{v}^{f j}\left\langle e_{i} g^{p c+d}, e_{j} \gamma^{p e+f}\right\rangle \\
& \left.=\sum_{c, d, e, f=0}^{q, r, s, t} C(c, d, e, f) v^{d f} \hat{v}^{d f} \zeta_{2}^{(p c+d)(p e+f)} \quad \text { (by Lemma 1.3. }\right) \\
& =\sum_{c, d, e, f=0}^{q, r, s, t} C(c, d, e, f)\left(v \hat{v} \zeta_{2}\right)^{d f} \zeta_{1}^{c f+e d},
\end{aligned}
$$

where

$$
C(c, d, e, f)=\left(\begin{array}{l}
q \\
c
\end{array}\right)\left(\begin{array}{l}
r \\
d
\end{array}\right)\left(\begin{array}{l}
s \\
e
\end{array}\right)\left(\begin{array}{l}
t \\
f
\end{array}\right)(-1)^{q-c}(-1)^{r-d}(-1)^{s-e}(-1)^{t-f} .
$$

Since $\hat{v}$ is so that $v \hat{v} \zeta_{2}=1$,

$$
\begin{aligned}
S & =\sum_{c, d, e, f=0}^{q, r, s, t}\left(\begin{array}{l}
q \\
c
\end{array}\right)\left(\begin{array}{l}
r \\
d
\end{array}\right)\left(\begin{array}{l}
s \\
e
\end{array}\right)\left(\begin{array}{l}
t \\
f
\end{array}\right)(-1)^{q-c}(-1)^{r-d}(-1)^{s-e}(-1)^{t-f} \zeta_{1}^{c f+e d} \\
& =\left(\sum_{c, f=0}^{q, t}\left(\begin{array}{l}
q \\
c
\end{array}\right)\left(\begin{array}{l}
t \\
f
\end{array}\right)(-1)^{q-c}(-1)^{t-f} \zeta_{1}^{c f}\right)\left(\sum_{d, e=0}^{r, s}\left(\begin{array}{l}
r \\
d
\end{array}\right)\left(\begin{array}{l}
s \\
e
\end{array}\right)(-1)^{r-d}(-1)^{s-e} \zeta_{1}^{e d}\right) .
\end{aligned}
$$

Suppose $q \geq t$. Then considering the left sum,

$$
\begin{aligned}
\sum_{c, f=0}^{q, t}\left(\begin{array}{l}
q \\
c
\end{array}\right)\left(\begin{array}{l}
t \\
f
\end{array}\right)(-1)^{q-c}(-1)^{t-f} \zeta_{1}^{c f} & =\sum_{f=0}^{t}\left(\begin{array}{l}
t \\
f
\end{array}\right)(-1)^{t-f}\left(\sum_{c=0}^{q}\left(\begin{array}{l}
q \\
c
\end{array}\right)(-1)^{q-c}\left(\zeta_{1}^{f}\right)^{c}\right) \\
& =\sum_{f=0}^{t}\left(\begin{array}{l}
t \\
f
\end{array}\right)(-1)^{t-f}\left(\zeta_{1}^{f}-1\right)^{q},
\end{aligned}
$$

and since $\left(\zeta_{1}-1\right)^{q}$ divides every term of the sum, the order of the left sum is at least

$$
q e^{\prime}=q i+q i^{\prime} \geq q i+t i^{\prime} .
$$

Since the same argument will work if $t \geq q$, and will also work with the right sum (involving $d$ and $e$ ), it follows that $S$ has order $\geq q i+t i^{\prime}+r j+s j^{\prime}$, as we wished to show. Therefore $A\left(j^{\prime}, i^{\prime}, \hat{v}\right)=A(i, j, v)^{*}$, and so $A(i, j, v)$ is an $R$-Hopf order, completing the proof. 
Remark 1.4. Consider the units $v$ and $\hat{v}$ of Theorem 1.2. Since ord $\left(\zeta_{2}-1\right)=e^{\prime} / p$, we have the following possibilities:

(1) If $\operatorname{ord}(\hat{v}-1)>e^{\prime} / p$, then $\operatorname{ord}(v-1)=e^{\prime} / p$.

(2) If $\operatorname{ord}(v-1)>e^{\prime} / p$, then $\operatorname{ord}(\hat{v}-1)=e^{\prime} / p$.

(3) If $\operatorname{ord}(v-1) \leq e^{\prime} / p$ and $\operatorname{ord}(\hat{v}-1) \leq e^{\prime} / p$, then $\operatorname{ord}(v-1)=\operatorname{ord}(\hat{v}-1)$.

The hypotheses of Theorem 1.2, namely, $\operatorname{ord}\left(v^{p}-1\right) \geq p i^{\prime}+j$ and $\operatorname{ord}\left(\zeta_{1} v^{p}-1\right)$ $\geq i^{\prime}+p j$, imply that $e^{\prime} \geq i^{\prime}+j / p$ and $e^{\prime} \geq i^{\prime} / p+j$. Thus by Lemma 3.3, $\operatorname{ord}(v-1) \geq i^{\prime}+j / p$ and $\operatorname{ord}(\hat{v}-1) \geq i^{\prime} / p+j$. We then have:

- In Case $1, e^{\prime} / p=\operatorname{ord}(v-1) \geq i^{\prime}+j / p$, hence $j^{\prime} / p \geq i^{\prime}$, the dual padic condition on $i$ and $j$. Also, ord $(\hat{v}-1) \geq \operatorname{ord}(v-1) \geq i^{\prime}+j / p$ and $\operatorname{ord}(\hat{v}-1) \geq i^{\prime} / p+j$, so $A\left(j^{\prime}, i^{\prime}, \hat{v}\right)$ is Greither.

- In Case $2, e^{\prime} / p=\operatorname{ord}(\hat{v}-1) \geq i^{\prime} / p+j$, hence $i \geq p j$, the p-adic condition on $i$ and $j$. Also, ord $(v-1) \geq \operatorname{ord}(\hat{v}-1) \geq i^{\prime} / p+j$ and $\operatorname{ord}(v-1) \geq i^{\prime}+j / p$, so $A(i, j, v)$ is Greither.

- In Case $3, e^{\prime} / p \geq \operatorname{ord}(v-1) \geq i^{\prime}+j / p$, hence $j^{\prime} / p \geq i^{\prime}$, and also $e^{\prime} / p \geq$ $\operatorname{ord}(\hat{v}-1) \geq i^{\prime} / p+j$, hence $i \geq p j$, and both the p-adic and dual p-adic conditions hold. Also, since $\operatorname{ord}(v-1)=\operatorname{ord}(\hat{v}-1)$, both $A(i, j, v)$ and $A\left(j^{\prime}, i^{\prime}, \hat{v}\right)$ are Greither.

Thus in Theorem $1.2 i$ and $j$ always satisfy either the p-adic or dual p-adic condition, and either $A(i, j, v)$ or $A\left(j^{\prime}, i^{\prime}, \hat{v}\right)$ is a Greither order.

Theorem 1.5. The construction of Theorem 1.2 yields every Hopf order in $K C_{p^{2}}$

Proof. In Un94 the first author proved that every Hopf order in $K C_{p^{2}}$ is either a Greither order or the dual of a Greither order. Thus it suffices to show that every Greither order $H(i, j, v)$, that is, an order of the form $A(i, j, v)$, where $i \geq p j$ and $v$ satisfies

$$
\operatorname{ord}(v-1) \geq i^{\prime}+j / p \text { and } \operatorname{ord}(v-1) \geq i^{\prime} / p+j,
$$

is of the form $A(i, j, v)$ in Theorem 1.2. For that we only need to show that if $i \geq p j$ and $v$ satisfies $\operatorname{ord}(v-1) \geq i^{\prime}+j / p$ and $\operatorname{ord}(v-1) \geq i^{\prime} / p+j$, then

$$
\operatorname{ord}\left(\zeta_{2} v-1\right) \geq i^{\prime} / p+j,
$$

so that the valuation hypotheses on $\zeta_{1} v^{p}-1$ and $v^{p}-1$ in Theorem 1.2 hold. If $\operatorname{ord}\left(\zeta_{2} v-1\right) \geq \operatorname{ord}(v-1)$, as in cases (1) and (3) of Remark 1.4, then (2) is clear. Otherwise, we are in case (2) of Remark 1.4, so the p-adic condition on $i$ and $j$ gives $i / p \geq j$, so $e^{\prime} / p \geq i^{\prime} / p+j$, and hence

$$
\operatorname{ord}\left(\zeta_{2} v-1\right) \geq i^{\prime} / p+j .
$$

Note also that in either case, $i \geq j$ (and, equivalently, $j^{\prime} \geq i^{\prime}$ ); cf. [Un94, Theorem 1.3.1].

We conclude this subsection on rank $p^{2}$ Hopf orders by looking at the relationship between general Hopf orders in $K C_{p^{2}}$ and Larson orders.

Let $A(i, j, v)$ be an $R$-Hopf order in $K C_{p^{2}}$. Then $A(i, j, v)$ contains a largest Larson order, denoted by $\mathcal{L}(A(i, j, v))$. Necessarily, $\mathcal{L}(A(i, j, v))=H(i, l)$, where $l=j$ if $\operatorname{ord}(1-v) \geq i^{\prime}+j$, and $l=i-e^{\prime}+\operatorname{ord}(1-v)$, that is, $\operatorname{ord}(1-v)=i^{\prime}+l$ otherwise ([Un94, Theorem 1.4.0]). 
Under certain conditions $A(i, j, v)$ also contains a "largest Larson dual", that is, the maximal $R$-Hopf order in $K C_{p^{2}}$ of the form $H(s, r)^{*}$ which is contained in $A(i, j, v)$. We denote this Hopf order by $\mathcal{L} \mathcal{D}(A(i, j, v))$. Assuming $K$ contains $\zeta_{2}$, if $H(s, r)^{*}$ is contained in $A(i, j, v)$, then $A(i, j, v)^{*}$ is contained in $H(s, r)$; hence the largest Larson dual in $A(i, j, v)$ is the dual of the smallest Larson order containing $A(i, j, v)^{*}=A\left(j^{\prime}, i^{\prime}, \hat{v}\right)$.

Lemma 1.6. Let $A(i, j, v)$ be an $R$-Hopf order in $K C_{p^{2}}$ and set $\hat{v}=v^{-1} \zeta_{2}^{-1}$.

If $j^{\prime} \geq p i^{\prime}$ and $\operatorname{ord}(\hat{v}-1) \geq i^{\prime}+j$, then $A(i, j, v)=H\left(j^{\prime}, i^{\prime}\right)^{*}$ is a Larson dual.

If $e^{\prime} / p \geq i^{\prime}$ and $\operatorname{ord}(\hat{v}-1)=i^{\prime}+\varrho$ with $\varrho<j$, then $\mathcal{L} \mathcal{D}(A(i, j, v))=H\left(\varrho^{\prime}, i^{\prime}\right)^{*}$.

Proof. The first case follows from remarks near the beginning of this subsection. For the case when $A(i, j, v)$ is not a Larson dual, we seek $\varrho^{\prime}$ minimal $\geq j^{\prime}$ so that $H\left(\varrho^{\prime}, i^{\prime}\right)$ is Larson, hence

$$
p i^{\prime} \leq \varrho^{\prime} \leq e^{\prime}
$$

and $A\left(j^{\prime}, i^{\prime}, \hat{v}\right) \subset A\left(\varrho^{\prime}, i^{\prime}, \hat{v}\right)=H\left(\varrho^{\prime}, i^{\prime}\right)$, hence

$$
\operatorname{ord}(1-\hat{v}) \geq i^{\prime}+\varrho \text {. }
$$

We let $\operatorname{ord}(1-\hat{v})=i^{\prime}+\varrho$ with $\varrho<j$, and show

$$
p i^{\prime} \leq \varrho^{\prime} \leq e^{\prime}
$$

If so, then $H\left(\varrho^{\prime}, i^{\prime}\right)$ is Larson and minimal containing $A\left(j^{\prime}, i^{\prime}, \hat{v}\right)$, so $H\left(\varrho^{\prime}, i^{\prime}\right)^{*}$ is the largest Larson dual contained in $A(i, j, v)$.

We consider the cases of Remark 1.4. In cases (1) and (3), we have $j^{\prime} \geq p i^{\prime}$, and since $\varrho^{\prime}>j^{\prime}$, we have $\varrho^{\prime} \geq p i^{\prime}$. Also,

$$
i^{\prime}+\varrho=\operatorname{ord}(\hat{v}-1) \geq \operatorname{ord}(v-1) \geq i^{\prime}+j / p,
$$

hence $\varrho \geq 0$, hence $\varrho^{\prime} \leq e^{\prime}$.

In case $(2), \operatorname{ord}(\hat{v}-1)=i^{\prime}+\varrho=e^{\prime} / p \geq i^{\prime}$ by hypothesis, so $\varrho \geq 0$; also,

$$
\begin{aligned}
\varrho^{\prime} & =i^{\prime}+e^{\prime}-e^{\prime} / p \\
& =i^{\prime}+\left(\frac{p-1}{p}\right) e^{\prime} \\
& \geq i^{\prime}+\left(\frac{p-1}{p}\right) p i^{\prime}=p i^{\prime} .
\end{aligned}
$$

Orders in $K C_{p^{3}}$. We first review the class of $R$-Hopf orders in $K C_{p^{3}}$ constructed in Un96.

Let $H$ be an arbitrary $R$-Hopf order in $K C_{p^{3}}$. Then $H$ induces the short exact sequences of Hopf orders

$$
R \rightarrow A(i, j, u) \rightarrow H \rightarrow H(k) \rightarrow R
$$

and

$$
R \rightarrow H(i) \rightarrow H \rightarrow A(j, k, w) \rightarrow R,
$$

where $A(i, j, u)$ and $A(j, k, w)$ are Hopf orders in $K C_{p^{2}}$, and $H(i)$ and $H(k)$ are Larson orders in $K C_{p}$. It follows that $H$ is of the form

$$
H=R\left[\frac{g^{p^{2}}-1}{\pi^{i}}, \frac{a_{u} g^{p}-1}{\pi^{j}}, \Upsilon\right],
$$


where $\Upsilon$ is some element of $H$ mapping to $\frac{a_{w} h-1}{\pi^{k}}$ in $A(j, k, w)$, where $h$ is $g$ $\left(\bmod \left\langle g^{p^{2}}\right\rangle\right)$. If $\Upsilon$ is of the form

$$
\frac{a_{v} b_{w} g-1}{\pi^{k}}
$$

where

$$
b_{w}=\sum_{0 \leq p a+b \leq p^{2}-1} w^{a} e_{p a+b}
$$

so that

$$
H=A(i, j, u)\left[\frac{a_{v} b_{w} g-1}{\pi^{k}}\right]=R\left[\frac{g^{p^{2}}-1}{\pi^{i}}, \frac{a_{u} g^{p}-1}{\pi^{j}}, \frac{a_{v} b_{w} g-1}{\pi^{k}}\right],
$$

then we call $H$ a triangular Hopf order. Here

$$
e_{p a+b}=\frac{1}{p^{2}} \sum_{r, s=0}^{p-1} \zeta_{2}^{-(p a+b)(p r+s)} g^{p(p r+s)}
$$

for $0 \leq a, b \leq p-1$ are the pairwise orthogonal minimal idempotents of $K C_{p^{2}}, C_{p^{2}}=$ $\left\langle g^{p}\right\rangle$. In what follows we shall denote the $R$-algebra $R\left[\frac{g^{p^{2}}-1}{\pi^{i}}, \frac{a_{u} g^{p}-1}{\pi^{j}}, \frac{a_{v} b_{w} g-1}{\pi^{k}}\right]$ by $H(i, j, k, u, v, w)$.

Analogous to the elements $a_{u}$, the elements $b_{w}$ are multiplicative:

$$
b_{y} b_{w}=b_{y w}
$$

(clear, since the $e_{p a+b}$ are idempotents). One may verify that $b_{w}$ maps to $a_{w}$ under the map from $H$ to $A(j, k, w)$.

In CU03] a Hopf order $H$ in $K C_{p^{3}}$ was called a cohomological Hopf order if

$$
H=A(i, j, u)\left[\frac{b_{v, w} g-1}{\pi^{k}}\right]
$$

where

$$
b_{v, w}=\sum_{0 \leq p a+b \leq p^{2}-1} v^{b} w^{p a+b} e_{p a+b} .
$$

Then $b_{w^{p}}=b_{w^{-1}, w}$, and since

$$
e_{b}^{1}=\sum_{a=0}^{p-1} e_{p a+b}^{2},
$$

where $e_{b}^{1}, b=0, \ldots, p-1$, are the minimal idempotents of $K C_{p}, C_{p}=\left\langle g^{p^{2}}\right\rangle$, and $e_{p a+b}^{2}=e_{p a+b}$, it follows easily that $b_{v, 1}=a_{v}$, and so

$$
b_{v, w}=b_{v w, 1} b_{w^{-1}, w}=a_{v w} b_{w^{p}} .
$$

Thus the cohomological Hopf orders of [CU03 are included in the class of triangular Hopf orders described above. Note that

$$
g^{p}=b_{1, \zeta_{2}}=b_{\zeta_{2}, 1} b_{\zeta_{2}^{-1}, \zeta_{2}}=a_{\zeta_{2}} b_{\zeta_{1}}
$$

We now introduce a collection of triangular Hopf orders constructed in Un96. $\S 4.1, \S 4.2]$.

Let $U(R)$ denote the group of units in $R$. Let $p i^{\prime} \leq j^{\prime}, H\left(j^{\prime}, i^{\prime}\right)$ be the Larson order in $K C_{p^{2}}$, and let $H\left(j^{\prime}, i^{\prime}\right)^{*}=A\left(i, j, \zeta_{2}^{-1}\right)$ be its linear dual. Let $H(k)$ denote a Larson order in $K C_{p}$ for which $p k \leq l$, where $H(i, l)=\mathcal{L}\left(H\left(j^{\prime}, i^{\prime}\right)^{*}\right)$ is the largest 
Larson order contained in $H\left(j^{\prime}, i^{\prime}\right)^{*}$. Then $l=j$ if $e^{\prime} / p \geq i^{\prime}+j$, and otherwise, $l=e^{\prime} / p-i^{\prime}\left(\geq 0\right.$ since $\left.p i^{\prime} \leq j^{\prime} \leq e^{\prime}\right)$. (Note that $p l=e^{\prime}-p i^{\prime}<e^{\prime}-i^{\prime}=i$, so $i$ and $l$ are p-adic.) Let

\section{$\operatorname{Ext}^{1}\left(\operatorname{Spec} H\left(j^{\prime}, i^{\prime}\right)^{*}, \operatorname{Spec} H(k)\right)$}

denote the collection of 1-extensions of Spec $H(k)$ by Spec $H\left(j^{\prime}, i^{\prime}\right)^{*}$.

Here is the main result of [Un96] (cf. [CU03, Theorem 4.0]):

Theorem 1.7. Assume $p i^{\prime} \leq j^{\prime}, p k \leq j$ and $i^{\prime}+p k \leq e^{\prime} / p$. Let $M$ be the group of pairs $(v, w) \in U(R) \times U(R)$ such that

(A) $\operatorname{ord}(v-1) \geq i^{\prime} / p+k$,

(B) $\operatorname{ord}(w-1) \geq j^{\prime} / p+k$,

(C) $\operatorname{ord}(w-1) \geq j^{\prime}+k / p$,

(D) $\operatorname{ord}\left(v^{p} w^{-1}-1\right) \geq p i^{\prime}+k$,

and let $N$ be the subgroup of $M$ consisting of pairs $(v, w)$ such that $\operatorname{ord}(v-1) \geq i^{\prime}+k$ and $\operatorname{ord}(w-1) \geq j^{\prime}+k$. Then the classes $[(v, w)]$ in $M / N$ are in 1-1 correspondence with elements in $\operatorname{Ext}^{1}\left(\operatorname{Spec} H\left(j^{\prime}, i^{\prime}\right)^{*}\right.$, Spec $\left.H(k)\right), p k \leq l$, which over $K$ appear as $\mu_{p, K} \longrightarrow \mu_{p^{3}, K} \longrightarrow \mu_{p^{2}, K}$. The class $[(v, w)]$ corresponds to a short exact sequence of $R$-Hopf orders

$$
\begin{gathered}
R \rightarrow H\left(j^{\prime}, i^{\prime}\right)^{*} \longrightarrow H\left(i, j, k, \zeta_{2}^{-1}, v, w\right) \\
=R\left[\frac{g^{p^{2}}-1}{\pi^{i}}, \frac{a_{\zeta_{2}^{-1}} g^{p}-1}{\pi^{j}}, \frac{a_{v} b_{w} g-1}{\pi^{k}}\right] \longrightarrow H(k) \rightarrow R .
\end{gathered}
$$

Since the Hopf orders of Theorem 1.7 were constructed by a cohomology argument extending that in Gr92, we shall call those Hopf orders cohomological Hopf orders.

The Hopf algebras in Theorem 1.7 are extensions of Larson orders by Larson duals, and involve five parameters: $i, j, k, v$ and $w$. We can induce from them a collection of "6-parameter" triangular Hopf orders in $K C_{p^{3}}$.

Define $\varrho^{\prime}$ by

$$
\left\{\begin{array}{l}
\varrho^{\prime}=i^{\prime}+e^{\prime}-\operatorname{ord}(\hat{u}-1) \text { if } \operatorname{ord}(\hat{u}-1)<i^{\prime}+j, \\
\varrho^{\prime}=j^{\prime} \text { if } \operatorname{ord}(\hat{u}-1) \geq i^{\prime}+j
\end{array}\right.
$$

and $\ell$ by

$$
\left\{\begin{array}{l}
e^{\prime} / p=\operatorname{ord}\left(\zeta_{2}-1\right)=i^{\prime}+\ell \text { if } e^{\prime} / p<i^{\prime}+\varrho, \\
\ell=\varrho \text { if } e^{\prime} / p \geq i^{\prime}+\varrho .
\end{array}\right.
$$

Proposition 1.8. Let $H(i, j, u)$ be a Hopf order with $i^{\prime} \leq e^{\prime} / p$. If $p k \leq \ell$ and $v, w$ satisfy

(A) $\operatorname{ord}(v-1) \geq i^{\prime} / p+k$,

(B) $\operatorname{ord}(w-1) \geq \varrho^{\prime} / p+k$,

(C) $\operatorname{ord}(w-1) \geq \varrho^{\prime}+k / p$, and

(D) $\operatorname{ord}\left(v^{p} w^{-1}-1\right) \geq p i^{\prime}+k$,

then $H\left(i, \varrho, k, \zeta_{2}^{-1}, v, w\right)$ is a cohomological Hopf order, and $H(i, j, k, u, v, w)$ is a triangular Hopf order.

Proof. To obtain $H\left(i, \varrho, k, \zeta_{2}^{-1}, v, w\right)$ we need only check the inequalities

$$
p i^{\prime} \leq \varrho^{\prime}, \quad p k \leq \varrho
$$


of Theorem 1.7. Since $e^{\prime} / p \geq i^{\prime}$, then by Lemma 1.6. $H(i, j, u)$ has a largest Larson dual,

$$
\mathcal{L D}(A(i, j, u))=H\left(\varrho^{\prime}, i^{\prime}\right)^{*}=H\left(i, \varrho, \zeta_{2}^{-1}\right),
$$

where $\varrho^{\prime} \geq p i^{\prime}$ by construction. Then the assumption $p k \leq \ell$ implies that $p k \leq \varrho$ by definition of $\ell$. Thus

$$
H\left(i, \varrho, k, \zeta_{2}^{-1}, v, w\right)=H\left(i, j, \zeta_{2}^{-1}\right)\left[\frac{a_{v} b_{w} g-1}{\pi^{k}}\right]
$$

is a Hopf order. Since $H\left(i, \varrho, \zeta_{2}^{-1}\right) \subset H(i, j, u)$, it is clear that

$$
H(i, j, k, u, v, w)=H(i, j, u)\left[\frac{a_{v} b_{w} g-1}{\pi^{k}}\right]
$$

is then a Hopf order, and there is an induced short exact sequence of Hopf orders

$$
R \rightarrow H(i, j, u) \rightarrow H(i, j, k, u, v, w) \rightarrow H(k) \rightarrow R .
$$

An $R$-Hopf order $H(i, j, k, u, v, w)$ arising in the manner of Proposition 1.8 will be called a Hopf order induced from a Larson dual, or, for short, an ILD order.

It is natural to ask whether all triangular Hopf orders are ILD orders as in Proposition 1.8. One goal in the remainder of this paper is to investigate this question. However, we shall soon show that under the restriction $e^{\prime} / p \geq i^{\prime}$, every triangular Hopf order is "induced from both ends" of an ILD Hopf order (Theorem $2.8)$.

\section{Duality lemmas}

In this section we collect together various results on duality needed in order to extend the construction of Theorem 1.2 to rank $p^{3}$ Hopf orders. We need two useful preliminary results.

Proposition 2.1. If $\operatorname{ord}(\hat{u}-1)=i^{\prime}+\nu^{\prime}>0, e^{\prime} \geq \nu^{\prime} \geq 0$, then

$$
R\left[\frac{a_{\hat{u}}-1}{\pi^{i^{\prime}}}\right]=H(\nu) \text {. }
$$

Proof. We know that $a_{\hat{u}}-1 \in \pi^{i^{\prime}} H(\nu)$ by (1), hence

$$
R\left[\frac{a_{\hat{u}}-1}{\pi^{i^{\prime}}}\right] \subset H(\nu) .
$$

To show equality, we compare discriminants. We have

$$
\operatorname{disc}(H(\nu))=\frac{p^{p}}{\pi^{p(p-1) \nu}} R
$$

by [Gr92, Lemma 1.3a]. So

$$
\operatorname{ord}\left(\operatorname{disc}(H(\nu))=p(p-1) e^{\prime}-p(p-1) \nu=p(p-1) \nu^{\prime} .\right.
$$


On the other hand,

$$
\begin{aligned}
\operatorname{disc}\left(R\left[\frac{a_{\hat{u}}-1}{\pi^{i^{\prime}}}\right]\right) & =\operatorname{disc}\left(1, \frac{a_{\hat{u}}-1}{\pi^{i^{\prime}}},\left(\frac{a_{\hat{u}}-1}{\pi^{i^{\prime}}}\right)^{2}, \ldots,\left(\frac{a_{\hat{u}}-1}{\pi^{i^{\prime}}}\right)^{p-1}\right) \\
& =\frac{1}{\pi^{i^{\prime} p(p-1)}} \operatorname{disc}\left(1, a_{\hat{u}}-1,\left(a_{\hat{u}}-1\right)^{2}, \ldots,\left(a_{\hat{u}}-1\right)^{p-1}\right) \\
& =\frac{1}{\pi^{i^{\prime} p(p-1)}} \operatorname{disc}\left(1, a_{\hat{u}}, a_{\hat{u}}^{2}, \ldots, a_{\hat{u}}^{p-1}\right) .
\end{aligned}
$$

Now $a_{\hat{u}}^{k}=\sum_{\ell=0}^{p-1} \hat{u}^{\ell k} e_{\ell}$ for $k=1, \ldots, p-1$. So

$$
\left(\begin{array}{c}
1 \\
a_{\hat{u}} \\
a_{\hat{u}}^{2} \\
\vdots \\
a_{\hat{u}}^{p-1}
\end{array}\right)=M\left(\begin{array}{c}
e_{0} \\
e_{1} \\
e_{2} \\
\vdots \\
e_{p-1}
\end{array}\right)
$$

where

Since

$$
M=\left(\begin{array}{ccccc}
1 & 1 & 1 & \ldots & 1 \\
1 & \hat{u} & \hat{u}^{2} & \ldots & \hat{u}^{p-1} \\
1 & \hat{u}^{2} & \hat{u}^{4} & \ldots & \hat{u}^{2(p-1)} \\
\vdots & \vdots & \vdots & \vdots & \vdots \\
1 & \hat{u}^{p-1} & \hat{u}^{2(p-1)} & \ldots & \hat{u}^{(p-1)^{2}}
\end{array}\right)
$$

$\operatorname{disc}\left(e_{0}, e_{1}, \ldots, e_{p-1}\right)=R$,

it suffices to compute $(\operatorname{det}(M))^{2}$. Since $M$ is Vandermonde,

$$
\operatorname{det}(M)=\prod_{0 \leq i<j \leq p-1}\left(\hat{u}^{j}-\hat{u}^{i}\right) .
$$

$\operatorname{But} \operatorname{ord}\left(\hat{u}^{j}-\hat{u}^{i}\right)=\operatorname{ord}\left(\hat{u}^{j-i}-1\right)=\operatorname{ord}(\hat{u}-1)$ since $\hat{u} \equiv 1 \bmod \pi R$. Thus

$$
\operatorname{ord}(\operatorname{det}(M))=\frac{p(p-1)}{2} \operatorname{ord}(\hat{u}-1)
$$

and so

$$
\begin{aligned}
\operatorname{ord}\left(\operatorname{disc} R\left[\frac{a_{\hat{u}}-1}{\pi^{i^{\prime}}}\right]\right) & =(p-1) p\left(i^{\prime}+\nu^{\prime}\right)-(p-1) p i^{\prime} \\
& =(p-1) p \nu^{\prime}=\operatorname{ord}(\operatorname{disc}(H(\nu)) .
\end{aligned}
$$

Hence $R\left[\frac{a_{\hat{u}}-1}{\pi^{i^{\prime}}}\right]=H(\nu)$, as we wished to prove.

Corollary 2.2. a) Let

$$
B=R\left[\frac{a_{x}-1}{\pi^{i}}, \frac{a_{y}-1}{\pi^{j}}\right] .
$$

If $\operatorname{ord}(x-1)=i+\mu^{\prime}>0, e^{\prime} \geq \mu^{\prime} \geq 0$ and $\operatorname{ord}(y-1)=j+\nu^{\prime}>0, e^{\prime} \geq \nu^{\prime} \geq 0$, then $B=H(\max \{\mu, \nu\})$.

b) If $\operatorname{ord}(\hat{u}-1)=i^{\prime}+\nu^{\prime}>0, e^{\prime} \geq \nu^{\prime} \geq 0, e^{\prime} \geq j \geq 0$, then

$$
R\left[\frac{a_{\hat{u}}-1}{\pi^{i^{\prime}}}, \frac{\sigma-1}{\pi^{j^{\prime}}}\right]=H(\lambda)
$$

with $\lambda=j^{\prime}$ if $\nu<j^{\prime}$, and $\lambda=\nu$ if $\nu \geq j^{\prime}$. 
Proof. Using Proposition 2.1, we have

$$
\begin{aligned}
B & =R\left[\frac{a_{x}-1}{\pi^{i}}\right]\left[\frac{a_{y}-1}{\pi^{j}}\right] \\
& =R\left[\frac{\sigma-1}{\pi^{\mu}}\right]\left[\frac{a_{y}-1}{\pi^{j}}\right] \\
& =R\left[\frac{a_{y}-1}{\pi^{j}}\right]\left[\frac{\sigma-1}{\pi^{\mu}}\right] \\
& =R\left[\frac{\sigma-1}{\pi^{\nu}}\right]\left[\frac{\sigma-1}{\pi^{\mu}}\right] \\
& =R\left[\frac{\sigma-1}{\pi^{\lambda}}\right],
\end{aligned}
$$

where $\lambda=\max \{\mu, \nu\}$.

Statement b) follows easily from a) since $\sigma=a_{\zeta_{1}}$.

In the next results we will use duality, and to keep track of which groups are involved, we shall subscript the duality brackets, as follows: $\langle,\rangle_{n}$ will denote the duality map $K C_{p^{n}} \times K \hat{C}_{p^{n}} \rightarrow K$ for $n=1,2,3$.

Our first duality results involve a "Gauss sum"defined in GC98, that arises in duality computations.

Let $x$ and $y$ be any units in $R$. The quantity

$$
G(x, y)=\frac{1}{p} \sum_{0 \leq i, j \leq p-1} \zeta_{1}^{-i j} x^{i} y^{j}
$$

is defined to be the Gauss sum of $x$ and $y([\overline{G C 98}])$. Note that $G(x, 1)=1$. Also,

$$
\begin{aligned}
G\left(\zeta_{1}^{k}, w\right) & =\frac{1}{p} \sum_{i, j=0}^{p-1} \zeta_{1}^{k i} \zeta_{1}^{-i j} w^{j} \\
& =\frac{1}{p} \sum_{j=0}^{p-1}\left(\sum_{i=0}^{p-1} \zeta_{1}^{(k-j) i}\right) w^{j}=w^{k}
\end{aligned}
$$

The Gauss sum arises in connection with duality because

$$
G(x, y)=\left\langle a_{x}, a_{y}\right\rangle
$$

(where $a_{x} \in K C_{p}, a_{y} \in K \hat{C}_{p}$ ), as is easily verified (cf. GC98]).

Proposition 2.3. Let $x, y$ be units in $R$ with $e^{\prime}>\operatorname{ord}(1-x), e^{\prime}>\operatorname{ord}(1-y)$, and $\operatorname{ord}(1-x)+\operatorname{ord}(1-y)>e^{\prime}$. Then

$$
\operatorname{ord}(G(x, y)-1)=\operatorname{ord}\left(\left\langle a_{x}, a_{y}\right\rangle_{1}-1\right)=\operatorname{ord}(1-x)+\operatorname{ord}(1-y)-e^{\prime} .
$$

Proof. Let $\operatorname{ord}(1-x)=n$ and $\operatorname{ord}(1-y)=n^{\prime}+s$, and suppose

$$
\operatorname{ord}\left(\left\langle a_{x}, a_{y}\right\rangle_{1}-1\right)=\operatorname{ord}\left(\left\langle a_{x}-1, a_{y}-1\right\rangle_{1}\right)=t .
$$

We have $a_{x}-1 \in H\left(n^{\prime}\right)$, and $a_{y}-1 \in \pi^{s} H(n)$ by (1). Thus

$$
\left\langle a_{x}-1, a_{y}-1\right\rangle_{1} \in \pi^{s} R
$$


hence $t \geq s$. Now

$$
\begin{aligned}
\left\langle\left(a_{x}-1\right)^{2}, a_{y}-1\right\rangle_{1} & =\left\langle\left(a_{x}-1\right)^{2}, a_{y}\right\rangle_{1} \\
& =\left(\left\langle a_{x}-1, a_{y}\right\rangle_{1}\right)^{2}+\left\langle\left(a_{x}-1\right) \otimes\left(a_{x}-1\right), \Delta\right\rangle_{1} \\
& =\left(\left\langle a_{x}-1, a_{y}-1\right\rangle_{1}\right)^{2}+\left\langle\left(a_{x}-1\right) \otimes\left(a_{x}-1\right), \Delta\right\rangle_{1},
\end{aligned}
$$

where

$$
\Delta=\Delta\left(a_{y}\right)-a_{y} \otimes a_{y} \in \pi^{p s} H(n) \otimes H(n)
$$

since $\operatorname{ord}\left(y^{p}-1\right)=p n^{\prime}+p s[\mathrm{C} 00,(31.10)]$. So

$$
\operatorname{ord}\left(\left\langle\left(a_{x}-1\right)^{2}, a_{y}-1\right\rangle_{1}\right) \geq \min \{2 t, p s\} .
$$

Then

$$
\begin{aligned}
& \left\langle\left(a_{x}-1\right)^{3}, a_{y}-1\right\rangle_{1} \\
& \quad=\left\langle\left(a_{x}-1\right)^{2}, a_{y}\right\rangle_{1}\left\langle a_{x}-1, a_{y}\right\rangle_{1}+\left\langle\left(a_{x}-1\right)^{2} \otimes\left(a_{x}-1\right), \Delta\right\rangle_{1}
\end{aligned}
$$

has order $\geq \min \{3 t, p s\}$, etc. So

$$
\left\langle R\left[a_{x}-1\right], \frac{\hat{a}_{y}-1}{\pi^{q}}\right\rangle_{1} \in R
$$

where $q=\min \{t, p s\} \geq s$. But $R\left[a_{x}-1\right]=H\left(n^{\prime}\right)$ by Proposition 2.1. Hence $\frac{\hat{a}_{y}-1}{\pi^{q}} \in H(n)$. Thus ord $(y-1) \geq n^{\prime}+q$ by (1). Since $q \geq s$ and $\operatorname{ord}(y-1)=n^{\prime}+s$, we have $s=t=q$. That completes the proof.

Lemma 2.4. Suppose $e^{\prime}>\operatorname{ord}\left(1-x_{i}\right), e^{\prime}>\operatorname{ord}(1-y)$, and $\operatorname{ord}\left(1-x_{i}\right)+\operatorname{ord}(1-y) \geq$ $e^{\prime}+s, s \geq 0, i=1,2$. Then

$$
G\left(x_{1} x_{2}, y\right) \equiv G\left(x_{1}, y\right) G\left(x_{2}, y\right) \quad\left(\bmod \pi^{p s} R\right) .
$$

Proof.

$$
\begin{aligned}
G\left(x_{1}, y\right) G\left(x_{2}, y\right)-G\left(x_{1} x_{2}, y\right) & =\left\langle a_{x_{1}}, a_{y}\right\rangle_{1}\left\langle a_{x_{2}}, a_{y}\right\rangle_{1}-\left\langle a_{x_{1}} a_{x_{2}}, a_{y}\right\rangle_{1} \\
& =\left\langle a_{x_{1}} \otimes a_{x_{2}}, a_{y} \otimes a_{y}\right\rangle_{1}-\left\langle a_{x_{1}} \otimes a_{x_{2}}, \Delta\left(a_{y}\right)\right\rangle_{1} \\
& =\left\langle a_{x_{1}} \otimes a_{x_{2}}, a_{y} \otimes a_{y}-\Delta\left(a_{y}\right)\right\rangle_{1} .
\end{aligned}
$$

Let $\operatorname{ord}(1-y)=n^{\prime}+s$. Then $\operatorname{ord}\left(1-x_{i}\right) \geq n$, so $a_{x_{i}} \in H\left(n^{\prime}\right), i=1,2$, and

$$
a_{y} \otimes a_{y}-\Delta\left(a_{y}\right) \in \pi^{p s} H(n) \otimes H(n) .
$$

Thus

$$
\left\langle a_{x_{1}} \otimes a_{x_{2}}, a_{y} \otimes a_{y}-\Delta\left(a_{y}\right)\right\rangle_{1} \in \pi^{p s} R .
$$

Corollary 2.5. Suppose $e^{\prime}>\operatorname{ord}(1-x) \geq n$ and $e^{\prime}>\operatorname{ord}(1-y) \geq n^{\prime}+s, s \geq 0$. Then for $0 \leq m \leq p-1$,

$$
G(x, y)^{m} \equiv G\left(x^{m}, y\right) \quad\left(\bmod \pi^{p s} R\right)
$$

and

$$
G\left(x \zeta_{1}^{m}, y\right) \equiv G(x, y) y^{m} \quad\left(\bmod \pi^{p s} R\right) .
$$

Proof. The first formula is an easy induction. The second is an immediate consequence of the property that $G\left(\zeta_{1}^{m}, y\right)=y^{m}$. 
Lemma 2.6 (Reduction Lemma). Let $v$ be an element of $K$, and let $\tau$ be the generator of $\hat{C}_{p^{2}}$ for which $\left\langle g^{p}, \tau\right\rangle_{2}=\zeta_{2}$. Then for all $0 \leq c, d \leq p-1$,

$$
\left\langle b_{y}, a_{v} \tau^{p c+d}\right\rangle_{2}=G\left(v \zeta_{1}^{c}, y\right)=\left\langle a_{v} a_{\zeta_{1}}^{c}, a_{y}\right\rangle_{1} .
$$

Proof.

$$
\begin{aligned}
\left\langle b_{y}, a_{v} \tau^{p c+d}\right\rangle_{2} & =\left\langle\sum_{m, n=0}^{p-1} y^{m} e_{p m+n}^{2}, a_{v} \tau^{p c+d}\right\rangle_{2} \\
& =\left\langle\sum_{m, n=0}^{p-1} y^{m} e_{p m+n}^{2}, \sum_{q=0}^{p-1} v^{q} e_{q}^{1} \tau^{p c+d}\right\rangle_{2} \\
& =\left\langle\sum_{m, n=0}^{p-1} y^{m} e_{p m+n}^{2}, \sum_{q=0}^{p-1} v^{q} \frac{1}{p} \sum_{r=0}^{p-1} \zeta_{1}^{-q r} \tau^{p r+p c+d}\right\rangle_{2} \\
& =\frac{1}{p} \sum_{m, n, q, r=0}^{p-1} v^{q} y^{m} \zeta_{1}^{-q r}\left\langle e_{p m+n}^{2}, \tau^{p r+p c+d}\right\rangle_{2} .
\end{aligned}
$$

Note that $\left\langle e_{p m+n}^{2}, \tau^{p r+p c+d}\right\rangle_{2}=1$ if $n=d$ and $m \equiv r+c \bmod p$, that is, if $r \equiv m-c$, and $\left\langle e_{p m+n}^{2}, \tau^{p r+p c+d}\right\rangle_{2}=0$ in all other cases. Thus

$$
\begin{aligned}
\left\langle b_{y}, a_{v} \tau^{p c+d}\right\rangle_{2} & =\frac{1}{p} \sum_{m, q=0}^{p-1} v^{q} \zeta_{1}^{-q(m-c)} y^{m} \\
& =\frac{1}{p} \sum_{m, q=0}^{p-1}\left(v \zeta_{1}^{c}\right)^{q} \zeta_{1}^{-q m} y^{m} \\
& =G\left(v \zeta_{1}^{c}, y\right) \\
& =\left\langle a_{v} a_{\zeta_{1}^{c}}, a_{y}\right\rangle_{1} .
\end{aligned}
$$

Using the Reduction Lemma, we can study when $b_{y}-1$ is in $\pi^{q} A(i, j, u)$, where $A(i, j, u)$ is an arbitrary $R$-Hopf order in $K C_{p^{2}}$.

Theorem 2.7. a) Let $y$ be a unit of $R$. Suppose $A(i, j, u)$ is a Hopf order and let $\hat{u}=\zeta_{2}^{-1} u^{-1}$ with $\operatorname{ord}(\hat{u}-1)=i^{\prime}+\nu^{\prime}>0$ with $e^{\prime} \geq \nu^{\prime} \geq 0$. Then $b_{y}-1$ is in $\pi^{q} A(i, j, u)$ iff $\operatorname{ord}(y-1) \geq \varrho^{\prime}+q$, where

$$
\begin{gathered}
\varrho^{\prime}=\nu \text { if } \nu^{\prime}<j \text { and } \\
\varrho^{\prime}=j^{\prime} \text { if } \nu^{\prime} \geq j .
\end{gathered}
$$

b) Suppose $\operatorname{ord}(y-1)<e^{\prime}$. Then $b_{y}-1 \in \pi^{q} A(i, j, u)$ if and only if $\operatorname{ord}(\hat{u}-1) \geq i^{\prime}$ and $\operatorname{ord}(y-1) \geq \varrho^{\prime}+q$.

Proof. We have $b_{y}-1 \in \pi^{q} A(i, j, u)$ iff

$$
\left\langle b_{y}-1,\left(\tau^{p}-1\right)^{r}\left(a_{\hat{u}} \tau-1\right)^{s}\right\rangle_{2} \in \pi^{q+r j^{\prime}+s i^{\prime}} R,
$$

for $0 \leq r, s \leq p-1$. If $r=s=0$, we have

$$
\left\langle b_{y}-1,\left(\tau^{p}-1\right)^{r}\left(a_{\hat{u}} \tau-1\right)^{s}\right\rangle_{2}=\left\langle b_{y}-1,1\right\rangle_{2}=0,
$$


so (3) holds iff

$$
\left\langle b_{y}-1,\left(\tau^{p}-1\right)^{r}\left(a_{\hat{u}} \tau-1\right)^{s}\right\rangle_{2} \in \pi^{q+r j^{\prime}+s i^{\prime}} R
$$

for $0<r+s$. Since

$$
\left\langle 1,\left(\tau^{p}-1\right)^{r}\left(a_{\hat{u}} \tau-1\right)^{s}\right\rangle_{2}=0
$$

for $r+s>0$, we have for $r+s>0$,

$$
\begin{aligned}
\left\langle b_{y}-1,\right. & \left.\left(\tau^{p}-1\right)^{r}\left(a_{\hat{u}} \tau-1\right)^{s}\right\rangle_{2} \\
& =\left\langle b_{y},\left(\tau^{p}-1\right)^{r}\left(a_{\hat{u}} \tau-1\right)^{s}\right\rangle_{2} \\
& =\sum_{c=0}^{r} \sum_{d=0}^{s}\left(\begin{array}{l}
r \\
c
\end{array}\right)\left(\begin{array}{l}
s \\
d
\end{array}\right)(-1)^{r-c}(-1)^{s-d}\left\langle b_{y}, \tau^{p c}\left(a_{\hat{u}} \tau\right)^{d}\right\rangle_{2} \\
& =\sum_{c=0}^{r} \sum_{d=0}^{s}\left(\begin{array}{l}
r \\
c
\end{array}\right)\left(\begin{array}{l}
s \\
d
\end{array}\right)(-1)^{r-c}(-1)^{s-d}\left\langle a_{\hat{u}}^{d} a_{\zeta_{1}}^{c}, a_{y}\right\rangle_{1},
\end{aligned}
$$

(by the Reduction Lemma 2.6)

$$
=\left\langle\left(a_{\hat{u}}-1\right)^{s}\left(a_{\zeta_{1}}-1\right)^{r}, a_{y}\right\rangle_{1} .
$$

Thus condition (3) is equivalent to the condition

$$
\left\langle\left(a_{\hat{u}}-1\right)^{s}\left(a_{\zeta_{1}}-1\right)^{r}, a_{y}\right\rangle_{1} \in \pi^{q+r j^{\prime}+s i^{\prime}} R
$$

or

$$
\left\langle\left(\frac{a_{\hat{u}}-1}{\pi^{i^{\prime}}}\right)^{s}\left(\frac{a_{\zeta_{1}}-1}{\pi^{j^{\prime}}}\right)^{r}, a_{y}\right\rangle_{1}=\left\langle\left(\frac{a_{\hat{u}}-1}{\pi^{i^{\prime}}}\right)^{s}\left(\frac{\sigma-1}{\pi^{j^{\prime}}}\right)^{r}, a_{y}\right\rangle_{1} \in \pi^{q} R,
$$

where $\sigma=g^{p^{2}}$ and $0 \leq r, s \leq p-1,0<r+s$. Since for all $0 \leq r, s \leq p-1$ with $0<r+s$, we have

$$
\left\langle\left(\frac{a_{\hat{u}}-1}{\pi^{i^{\prime}}}\right)^{s}\left(\frac{\sigma-1}{\pi^{j^{\prime}}}\right)^{r}, 1\right\rangle_{1}=0
$$

and since $\left\langle 1, a_{y^{p}}-1\right\rangle=0$, (3) is equivalent to

$$
\left\langle\left(\frac{a_{\hat{u}}-1}{\pi^{i^{\prime}}}\right)^{s}\left(\frac{\sigma-1}{\pi^{j^{\prime}}}\right)^{r}, a_{y}-1\right\rangle_{1} \in \pi^{q} R
$$

for all $0 \leq r, s \leq p-1$. Assume $\operatorname{ord}(\hat{u}-1)=i^{\prime}+\nu^{\prime}>0, e^{\prime} \geq \nu^{\prime} \geq 0$. Then by Corollary $2.2 \mathrm{~b})$,

$$
R\left[\frac{a_{\hat{u}}-1}{\pi^{i^{\prime}}}, \frac{\sigma-1}{\pi^{j^{\prime}}}\right]=H\left(\varrho^{\prime}\right)
$$

for $\varrho^{\prime}=\nu$ if $\nu^{\prime}<j$, and $\varrho^{\prime}=j^{\prime}$ if $\nu^{\prime} \geq j$. Thus (4) is equivalent to $a_{y}-1 \in \pi^{q} H(\varrho)$, which by (1) is equivalent to ord $(y-1) \geq \varrho^{\prime}+q$, giving a).

For b): It suffices to show, assuming ord $(y-1)<e^{\prime}$, that if $b_{y}-1$ is in $\pi^{q} A(i, j, u)$, then $\operatorname{ord}(\hat{u}-1)=i^{\prime}+\nu^{\prime} \geq i^{\prime}$ and $\operatorname{ord}(y-1) \geq j^{\prime}+q \geq \varrho^{\prime}+q$.

Since $b_{y}-1$ is in $\pi^{q} A(i, j, u)$, then for all $0 \leq r, s \leq p-1$ with $r+s>0$,

$$
\left\langle\left(a_{\hat{u}}-1\right)^{s}(\sigma-1)^{r}, a_{y}\right\rangle \in \pi^{q+s i^{\prime}+r j^{\prime}} R .
$$

In particular, for $s=0, r=1$ we have

$$
y-1=\left\langle\sigma-1, a_{y}\right\rangle \in \pi^{q+j^{\prime}} R,
$$

hence $\operatorname{ord}(y-1) \geq j^{\prime}+q$. Also, for $s=1, r=0$ we have

$$
\left\langle a_{\hat{u}}-1, a_{y}\right\rangle=G(\hat{u}, y)-1 \in \pi^{q+i^{\prime}} R \text {. }
$$


Since $A(i, j, u)$ is a Hopf order, by Theorem [1.5 we have ord $(\hat{u}-1) \geq i^{\prime} / p+j$. Hence

$$
\operatorname{ord}(\hat{u}-1)+\operatorname{ord}(y-1) \geq j^{\prime}+q+i^{\prime} / p+j>e^{\prime} .
$$

Assume ord $(y-1)<e^{\prime}$. If $\operatorname{ord}(\hat{u}-1) \geq e^{\prime}$, then $\operatorname{ord}(\hat{u}-1) \geq i^{\prime}$. Otherwise, by Proposition 2.3

$$
\operatorname{ord}(G(\hat{u}, y)-1)+e^{\prime}=\operatorname{ord}(y-1)+\operatorname{ord}(\hat{u}-1),
$$

hence

$$
\operatorname{ord}(y-1)+\operatorname{ord}(\hat{u}-1) \geq e^{\prime}+i^{\prime}+q .
$$

Since $\operatorname{ord}(y-1)<e^{\prime}$, we have $\operatorname{ord}(\hat{u}-1) \geq i^{\prime}+q \geq i^{\prime}$. Since $\varrho^{\prime} \leq j^{\prime}$ we have $\operatorname{ord}(y-1) \geq j^{\prime}+q \geq \varrho^{\prime}+q$, proving b).

Using Theorem 2.7 one can show that if $e^{\prime} / p \geq i^{\prime}$, then every triangular Hopf order is induced from "both ends" of an ILD Hopf order.

Theorem 2.8. Let $H=A(i, j, u)\left[\frac{a_{v} b_{w} g-1}{\pi^{k}}\right]$ be a triangular Hopf order with $e^{\prime} / p \geq$ $i^{\prime}$ and $a_{v}, b_{w}$ elements of $A(i, j, u), v, w \in U(R)$. Then there exists $\mu \leq k$ so that

$$
H^{\prime}=A(i, j, u)\left[\frac{a_{v} b_{w} g-1}{\pi^{\mu}}\right]
$$

is an ILD order.

Proof. If $e^{\prime} / p \geq i^{\prime}$, then $\operatorname{ord}(\hat{u}-1) \geq i^{\prime}$ : referring to Remark 1.4, this is clear in cases (1) and (2), where ord $(\hat{u}-1) \geq i^{\prime}$; in case $(3)$, ord $(\hat{u}-1)=\operatorname{ord}(u-1) \geq i^{\prime}+j / p$. Now since $a_{v} \in A(i, j, u)$, we have $a_{v} \in H(i)$, hence by $(1)$, ord $(v-1) \geq i^{\prime}$. Now by Theorem 2.7a), since $b_{w} \in A(i, j, u)$, we have $\operatorname{ord}(w-1) \geq \varrho^{\prime}$, where $\varrho^{\prime}=j^{\prime}$ if $\operatorname{ord}(\hat{u}-1) \geq i^{\prime}+j$, and otherwise ord $(\hat{u}-1)=i^{\prime}+\varrho$. The largest Larson dual in $A(i, j, u)$ is $H\left(\varrho^{\prime}, i^{\prime}\right)^{*}$, and $\varrho^{\prime} \geq p i^{\prime}$. We show that for some $\mu \leq k$, the triangular Hopf order

$$
H_{0}=H\left(\varrho^{\prime}, i^{\prime}\right)^{*}\left[\frac{a_{v} b_{w} g-1}{\pi^{\mu}}\right]
$$

satisfies the conditions of Theorem 1.7, namely:

(A) $\operatorname{ord}(v-1) \geq i^{\prime} / p+\mu$;

(B) $\operatorname{ord}(w-1) \geq \varrho^{\prime} / p+\mu$;

(C) $\operatorname{ord}(w-1) \geq \varrho^{\prime}+\mu / p$;

(D) $\operatorname{ord}\left(v^{p} w^{-1}-1\right) \geq p i^{\prime}+\mu$, with $p \mu \leq l, H(i, l)=\mathcal{L}\left(H\left(\varrho^{\prime}, i^{\prime}\right)^{*}\right)$.

Since $\operatorname{ord}(v-1) \geq i^{\prime}$ and $\varrho^{\prime} \geq p i^{\prime}$, we have

$$
\operatorname{ord}\left(v^{p} w^{-1}-1\right) \geq \min \left\{\operatorname{ord}\left(v^{p}-1\right), \operatorname{ord}(w-1)\right\} \geq p i^{\prime} .
$$

So let

$$
\operatorname{ord}\left(v^{p} w^{-1}-1\right)=p i^{\prime}+\mu_{D}
$$

Since $\operatorname{ord}(w-1) \geq \varrho^{\prime} / p$, let

$$
\operatorname{ord}(w-1)=\varrho^{\prime} / p+\mu_{B}=\varrho^{\prime}+\mu_{C} / p .
$$

Since $\operatorname{ord}(v-1) \geq i^{\prime}$, let $\operatorname{ord}(v-1)=i^{\prime} / p+\mu_{A}$. Finally, let $\mu$ satisfy

$$
0 \leq \mu \leq \min \left\{k, \mu_{A}, \mu_{B}, \mu_{C}, \mu_{D}, l / p\right\} .
$$

Then $p \mu \leq l$ and all of the inequalities (A)-(D) hold, so $H^{\prime}$ is an ILD Hopf order. 


\section{Duality Hopf orders}

We begin this section by isolating the main idea of Theorem 1.2

Theorem 3.1. Let $K$ contain a primitive $p^{n}$ th root of unity. Let $G$ be cyclic of order $p^{n}$ with character group $\hat{G}$. Suppose $H$ is an order over $R$ in $K G$ and $J$ is an order over $R$ in $K \hat{G}$. If $\langle J, H\rangle \subset R$ and $\operatorname{disc}\left(H^{*}\right)=\operatorname{disc}(J)$, then $J=H^{*}$ and both are Hopf orders.

Proof. It is well known that the two hypotheses,

$$
\langle J, H\rangle \subset R \text { and } \operatorname{disc}\left(H^{*}\right)=\operatorname{disc}(J),
$$

imply that $J=H^{*}$. But if $J=H^{*}$ and $J$ is an order over $R$ in $K \hat{G}=K G^{*}$, hence an $R$-algebra with operations induced from $K G^{*}$, then $J^{*}=H$ is an $R$-coalgebra with operations induced from $K G$, and hence $H$ is a bialgebra. Since $H$ is also closed under the inverse map, $H$ is a Hopf order. Then $H^{*}=J$ is also a Hopf order.

Using Theorem 3.1 we will construct triangular Hopf orders using a duality argument, a generalization to Hopf orders in $K C_{p^{3}}$ of the construction we presented for $K C_{p^{2}}$ as Theorem 1.2, Before doing so, we note some lemmas:

Lemma 3.2. Let $i^{\prime}>0$. Then $a_{u}$ is a unit of $H(i)$ iff $u-1 \in \pi^{i^{\prime}} R$.

Proof. We have $a_{u}$ in $H(i)$ iff $a_{u}-1 \in H(i)$, iff $u-1 \in \pi^{i^{\prime}} R$ by (1). But then $u$ is a unit of $R$ and $u^{-1}-1 \in \pi^{i^{\prime}} R$, so $a_{u^{-1}} \in H(i)$, and $a_{u} a_{u^{-1}}=a_{1}=1$.

Lemma 3.3. If $q \leq e^{\prime}$, then for $x$ in $R$, ord $\left(x^{p}-1\right) \geq p q$ if and only if $\operatorname{ord}(x-1) \geq$ $q$.

Proof. If $\operatorname{ord}(x-1) \geq q, q \leq e^{\prime}$, then ord $\left((1-x)^{p}\right) \geq p q$. By the binomial theorem, $(x-1)^{p}=x^{p}-1+W$, where $W$ is so that $\operatorname{ord}(W)=e+q$. Now if $e+q>\operatorname{ord}\left(x^{p}-1\right)$, then $\operatorname{ord}\left(x^{p}-1\right) \geq p q$. Otherwise, if $e+q \leq \operatorname{ord}\left(x^{p}-1\right)$, then $\operatorname{ord}\left(x^{p}-1\right) \geq p q$ since $e^{\prime} \geq q$. Conversely, suppose ord $\left(x^{p}-1\right) \geq p q, e^{\prime} \geq q$. If $e+q \geq \operatorname{ord}\left(x^{p}-1\right)$, then ord $(x-1) \geq q$. On the other hand, if $e+q<\operatorname{ord}\left(x^{p}-1\right)$, then $\operatorname{ord}\left((x-1)^{p}\right) \geq e+q \geq p q$, hence $\operatorname{ord}(x-1) \geq q$.

The next lemma is a routine computation, analogous to Lemma 1.3. using the fundamental duality relation $\left\langle g^{m}, \gamma^{n}\right\rangle_{3}=\zeta_{3}^{m n}$.

Lemma 3.4. Let $G=C_{p^{3}}=\langle g\rangle, \hat{G}=\langle\gamma\rangle$, and let $e_{p a+b}^{2}, \hat{e}_{p m+n}^{2}, a, b, m, n=$ $0, \ldots, p-1$, denote the idempotents in the maximal integral orders in $K\left[g^{p}\right]$ and $K\left[\gamma^{p}\right]$, respectively. Then for all $0 \leq c, d, e, \alpha, \beta, \delta \leq p-1$,

$$
\left\langle e_{p a+b} g^{p^{2} c+p d+e}, \hat{e}_{p m+n} \gamma^{p^{2} \alpha+p \beta+\delta}\right\rangle_{3}=\frac{1}{p} \zeta_{1}^{-(m-d)(a-\beta)} \zeta_{3}^{\left(p^{2} c+p d+e\right)\left(p^{2} \alpha+p \beta+\delta\right)},
$$

if $n=e$ and $b=\delta$, and 0 in all other cases.

Assume that $K$ contains $\zeta_{3}$, a primitive $p^{3}$ rd root of unity. Let $\gamma$ generate $\hat{C}_{p^{3}}$, the character group of $C_{p^{3}}$, so that $\langle\gamma, g\rangle_{3}=\zeta_{3}$. To begin the duality construction, let $A=A(i, j, u), i^{\prime}>0$, be an $R$-Hopf order in $K\left\langle g^{p}\right\rangle$, and for $v, w \in U(R)$, let

$$
H=A(i, j, k, u, v, w)=A\left[\frac{a_{v} b_{w} g-1}{\pi^{k}}\right],
$$


where, recall,

$$
a_{v} b_{w}=\sum_{l=0}^{p-1} v^{l} e_{l}^{1} \cdot \sum_{m, n=0}^{p-1} w^{m} e_{p m+n}^{2}=\sum_{m, n=0}^{p-1} v^{n} w^{m} e_{p m+n}^{2} .
$$

Let $B=A\left(k^{\prime}, j^{\prime}, z\right), k>0$, be an $R$-Hopf order in $K\left\langle\gamma^{p}\right\rangle$, and let

$$
J=A\left(k^{\prime}, j^{\prime}, i^{\prime}, z, x, y\right)=B\left[\frac{a_{x} b_{y} \gamma-1}{\pi^{i^{\prime}}}\right],
$$

where $x, y \in U(R)$. We note that if $H$ is a Hopf order, then both $A(i, j, u)$ and $A(j, k, w)$, the image of $H$ under the map sending $g^{p^{2}}$ to 1 , are Hopf orders, and hence necessarily $i \geq j \geq k$ (cf. Remark 1.4). So we assume $i \geq j \geq k$.

We wish to find conditions on $u, v, w, x, y, z$ in order that $H$ and $J$ are $R$-algebras and $\langle H, J\rangle_{3} \subset R$. Once we do so, then since the discriminants of $H$ and $J$ depend only on $i, j, k$ (cf. the proof of Theorem 1.2), it is routine to see that $\operatorname{disc}(J)=$ $\operatorname{disc}\left(H^{*}\right)$ and so $J=H^{*}$.

First we find conditions for $H$ to be an $R$-order. For this we want

- $A$ is a Hopf order, free of rank $p$ over the Larson order $H(i)$.

By Theorem 1.2 this is true if $a_{u}$ is a unit of $H(i)$, which is equivalent to

- $u-1 \in \pi^{i^{\prime}} R$, by Lemma 3.2, and also

- $u^{p}-1 \in \pi^{p i^{\prime}+j} R$; and

- $u^{p} \zeta_{1}-1 \in \pi^{i^{\prime}+p j} R$.

If $u^{p}-1 \in \pi^{p i^{\prime}+j} R$, then since $j \leq i$, Lemma 3.3 gives ord $(u-1) \geq i^{\prime}+j / p$, hence $u-1 \in \pi^{i^{\prime}} R$ follows from the other conditions.

- $H$ is an $R$-algebra, free of rank $p$ over $A$.

By [C00, (31.1)], this is true iff $\left(a_{v} b_{w}\right)^{p} g^{p}-1 \in \pi^{p k} A(i, j, u)$. Note that

$$
\begin{aligned}
\left(a_{v} b_{w} g\right)^{p} & =a_{v^{p}} b_{w^{p}} g^{p} \\
& =\sum_{a, b=0}^{p-1}\left(v^{p}\right)^{b}\left(w^{p}\right)^{a} e_{p a+b} g^{p} \\
& =\sum_{a, b=0}^{p-1}\left(v^{p}\right)^{b}\left(w^{p}\right)^{a} \zeta_{2}^{p a+b} e_{p a+b} \\
& =a_{v^{p} \zeta_{2}} b_{w^{p}} \zeta_{1} .
\end{aligned}
$$

Then $a_{v^{p} \zeta_{2}} b_{w^{p} \zeta_{1}}-1 \in \pi^{p k} A(i, j, u)$ if

$$
a_{v^{p} \zeta_{2}}-1 \in \pi^{p k} H(i)
$$

and

The first holds iff

$$
b_{w^{p} \zeta_{1}}-1 \in \pi^{p k} A(i, j, u)
$$

$$
\operatorname{ord}\left(v^{p} \zeta_{2}-1\right) \geq i^{\prime}+p k
$$

and if $\operatorname{ord}(\hat{u}-1) \geq i^{\prime}$, the second holds iff

$$
\begin{gathered}
\operatorname{ord}\left(w^{p} \zeta_{1}-1\right)+\operatorname{ord}(\hat{u}-1) \geq i^{\prime}+e^{\prime}+p k \text { if } \operatorname{ord}(\hat{u}-1)<i^{\prime}+j, \\
\operatorname{ord}\left(w^{p} \zeta_{1}-1\right) \geq j^{\prime}+p k \text { if } \operatorname{ord}(\hat{u}-1) \geq i^{\prime}+j
\end{gathered}
$$

by Theorem 2.7a). 
We collect these conditions into

Proposition 3.5. The algebra

$$
H=A(i, j, k, u, v, w)=R\left[\frac{g^{p^{2}}-1}{\pi^{i}}, \frac{a_{u} g^{p}-1}{\pi^{j}}, \frac{a_{v} b_{w} g-1}{\pi^{k}}\right]
$$

is free of rank $p$ over the Hopf order $A(i, j, u)$ if $i \geq j \geq k$ and the following inequalities hold:

(i) $\operatorname{ord}(u-1) \geq i^{\prime}+j / p$;

(ii) $\operatorname{ord}(\hat{u}-1) \geq i^{\prime} / p+j$;

(iii) $\operatorname{ord}\left(v^{p} \zeta_{2}-1\right) \geq i^{\prime}+p k$;

(iv) $\operatorname{ord}(\hat{u}-1) \geq i^{\prime}>0$;

(v) $\operatorname{ord}\left(w \zeta_{2}-1\right) \geq j^{\prime} / p+k$;

(vi) $\operatorname{ord}(\hat{u}-1)+\operatorname{ord}\left(w^{p} \zeta_{1}-1\right) \geq e^{\prime}+i^{\prime}+p k$.

Similarly, we have

Proposition 3.6. The algebra

$$
J=A\left(k^{\prime}, j^{\prime}, i^{\prime}, z, x, y\right)=R\left[\frac{g^{p^{2}}-1}{\pi^{k^{\prime}}}, \frac{a_{z} g^{p}-1}{\pi^{j^{\prime}}}, \frac{a_{x} b_{y} g-1}{\pi^{i^{\prime}}}\right]
$$

is free of rank $p$ over the Hopf order $A\left(k^{\prime}, j^{\prime}, z\right)$ if $k^{\prime} \geq j^{\prime} \geq i^{\prime}$ and the following inequalities hold:

(i) $\operatorname{ord}(z-1) \geq k+j^{\prime} / p$;

(ii) $\operatorname{ord}(\hat{z}-1) \geq k / p+j^{\prime}$;

(iii) $\operatorname{ord}\left(x^{p} \zeta_{2}-1\right) \geq k+p i^{\prime}$;

(iv) $\operatorname{ord}(\hat{z}-1) \geq k>0$;

(v) $\operatorname{ord}\left(y \zeta_{2}-1\right) \geq j / p+i^{\prime}$;

(vi) $\operatorname{ord}(\hat{z}-1)+\operatorname{ord}\left(y^{p} \zeta_{1}-1\right) \geq e^{\prime}+k+p i^{\prime}$.

We are left with choosing relations among $u, v, w, x, y, z$ and conditions so that $\langle J, H\rangle \subset R$. Here is the result.

Theorem 3.7. Suppose $i \geq j \geq k$. Consider the following valuation inequalities:

(i) $e^{\prime}>\operatorname{ord}(\hat{u}-1) \geq i^{\prime} / p+j$;

(ii) $\operatorname{ord}\left(v^{p} \zeta_{2}-1\right) \geq i^{\prime}+p k$;

(iii) $\operatorname{ord}(\hat{u}-1) \geq i^{\prime}>0$;

(iv) $\operatorname{ord}(\hat{u}-1)+\operatorname{ord}\left(w^{p} \zeta_{1}-1\right) \geq e^{\prime}+i^{\prime}+p k$;

(v) $\operatorname{ord}\left(w \zeta_{2}-1\right)=\operatorname{ord}(z-1) \geq j^{\prime} / p+k$;

(vi) $\operatorname{ord}(u-1)=\operatorname{ord}(\hat{y}-1) \geq i^{\prime}+j / p$;

(vii) $e^{\prime}>\operatorname{ord}(\hat{z}-1) \geq k / p+j^{\prime}$

(viii) $\operatorname{ord}\left(x^{p} \zeta_{2}-1\right) \geq k+p i^{\prime}$

(ix) $\operatorname{ord}(\hat{z}-1) \geq k>0$;

(x) $\operatorname{ord}(\hat{z}-1)+\operatorname{ord}\left(y^{p} \zeta_{1}-1\right) \geq e^{\prime}+k+p i^{\prime}$; and

(xi) $\operatorname{ord}(\hat{z}-1)+\operatorname{ord}(\hat{u}-1) \geq e^{\prime}+\left(\frac{p-1}{p}\right)\left(i^{\prime}+k+e^{\prime}\right)$.

Then $H=A(i, j, k, u, v, w)$ is an R-algebra, free over the $R$-Hopf order $A(i, j, u)$, if inequalities (i)-(vi) hold; $J=A\left(k^{\prime}, j^{\prime}, i^{\prime}, z, x, y\right)$ is an $R$-algebra, free over the $R$ Hopf order $A\left(k^{\prime}, j^{\prime}, z\right)$, if inequalities $(\mathrm{v})-(\mathrm{x})$ hold; , and $H$ and $J$ are dual triangular Hopf orders in $K C_{p^{3}}$ if $\hat{z}=w, \hat{u}=y, v x \zeta_{3} G(\hat{u}, \hat{z})=1$ and inequality (xi) holds. 
Proof. Since it is easy to verify that $H$ and $J$ are closed under the antipode (inverse) map on $K G$, to finish the proof we need to show that $\langle J, H\rangle \subset R$. To do this, we require that

$$
\begin{gathered}
\operatorname{ord}\left(\left\langle\left(g^{p^{2}}-1\right)^{q}\left(a_{u} g^{p}-1\right)^{r}\left(a_{v} b_{w} g-1\right)^{s},\left(\gamma^{p^{2}}-1\right)^{\sigma}\left(a_{z} \gamma^{p}-1\right)^{\tau}\left(a_{x} b_{y} \gamma-1\right)^{\varepsilon}\right\rangle_{3}\right) \\
\geq q i+r j+s k+\sigma k^{\prime}+\tau j^{\prime}+\varepsilon i^{\prime}
\end{gathered}
$$

for $q, r, s, \sigma, \tau, \varepsilon=0, \ldots, p-1$. Put

$$
\eta=\left\langle\left(g^{p^{2}}-1\right)^{q}\left(a_{u} g^{p}-1\right)^{r}\left(a_{v} b_{w} g-1\right)^{s},\left(\gamma^{p^{2}}-1\right)^{\sigma}\left(a_{z} \gamma^{p}-1\right)^{\tau}\left(a_{x} b_{y} \gamma-1\right)^{\varepsilon}\right\rangle_{3} .
$$

Then

$$
\begin{aligned}
\eta & =\sum_{c, d, e, \alpha, \beta, \delta=0}^{q, r, s, \sigma, \tau, \varepsilon} C\left\langle g^{p^{2} c} a_{u^{d}} g^{p d} a_{v^{e}} b_{w^{e}} g^{e}, \gamma^{p^{2} \alpha} a_{z^{\beta}} \gamma^{p \beta} a_{x^{\delta}} b_{y^{\delta}} \gamma^{\delta}\right\rangle_{3} \\
& =\sum_{c, r, s, \sigma, \tau, \varepsilon} C\left\langle g^{p^{2} c+p d+e} a_{u^{d} v^{e}} b_{w^{e}}, \gamma^{p^{2} \alpha+p \beta+\delta, \beta, \delta=0} a_{z^{\beta} x^{\delta}} b_{y^{\delta}}\right\rangle_{3} \\
& =\sum_{c, d, e, \alpha, \beta, \delta=0}^{q, r, s, \tau, \varepsilon} C(c, d, e, \alpha, \beta, \delta) \Gamma,
\end{aligned}
$$

where

$$
\begin{gathered}
C=C(c, d, e, \alpha, \beta, \delta) \\
=\left(\begin{array}{l}
q \\
c
\end{array}\right)\left(\begin{array}{l}
r \\
d
\end{array}\right)\left(\begin{array}{l}
s \\
e
\end{array}\right)\left(\begin{array}{l}
\sigma \\
\alpha
\end{array}\right)\left(\begin{array}{l}
\tau \\
\beta
\end{array}\right)\left(\begin{array}{l}
\varepsilon \\
\delta
\end{array}\right)(-1)^{q-c}(-1)^{r-d}(-1)^{s-e}(-1)^{\sigma-\alpha}(-1)^{\tau-\beta}(-1)^{\varepsilon-\delta}
\end{gathered}
$$

and

$$
\Gamma=\sum_{p a+b, p m+n=0}^{p^{2}-1} u^{d b} v^{e b} w^{a e} z^{\beta n} x^{\delta n} y^{m \delta}\left\langle e_{p a+b} g^{p^{2} c+p d+e}, \hat{e}_{p m+n} \gamma^{p^{2} \alpha+p \beta+\delta}\right\rangle_{3} .
$$

By Lemma 3.4.

$$
\left\langle e_{p a+b} g^{p^{2} c+p d+e}, \hat{e}_{p m+n} \gamma^{p^{2} \alpha+p \beta+\delta}\right\rangle_{3}=\frac{1}{p} \zeta_{1}^{-(m-d)(a-\beta)} \zeta_{3}^{\left(p^{2} c+p d+e\right)\left(p^{2} \alpha+p \beta+\delta\right)} \delta_{e, n} \delta_{b, \delta}
$$

so since $u y \zeta_{2}=1=w z \zeta_{2}$ and $v x \zeta_{3} G(w, y)=1$, we have

$$
\begin{aligned}
\Gamma & =\frac{1}{p} \sum_{a, m=0}^{p-1} u^{d \delta} v^{e \delta} w^{a e} z^{\beta e} x^{\delta e} y^{m \delta} \zeta_{1}^{-(m-d)(a-\beta)} \zeta_{3}^{\left(p^{2} c+p d+e\right)\left(p^{2} \alpha+p \beta+\delta\right)} \\
& =u^{d \delta} \zeta_{2}^{d \delta} z^{\beta e} \zeta_{2}^{\beta e}\left(v x \zeta_{3}\right)^{e \delta} \zeta_{1}^{c \delta+d \beta+e \alpha} \frac{1}{p} \sum_{a, m=0}^{p-1} y^{m \delta} w^{a e} \zeta_{1}^{-(m-d)(a-\beta)} \\
& =y^{-d \delta} w^{-\beta e}\left(v x \zeta_{3}\right)^{e \delta} \zeta_{1}^{c \delta+e \alpha} \frac{1}{p} \sum_{a, m=0}^{p-1}\left(y^{\delta} \zeta_{1}^{\beta}\right)^{m} \zeta_{1}^{-a m}\left(w^{e} \zeta_{1}^{d}\right)^{a} \\
& =y^{-d \delta} w^{-\beta e}\left(v x \zeta_{3}\right)^{e \delta} \zeta_{1}^{c \delta+e \alpha} G\left(y^{\delta} \zeta_{1}^{\beta}, w^{e} \zeta_{1}^{d}\right) \\
& =y^{-d \delta} w^{-\beta e} G(y, w)^{-e \delta} \zeta_{1}^{c \delta+e \alpha} G\left(y^{\delta} \zeta_{1}^{\beta}, w^{e} \zeta_{1}^{d}\right) .
\end{aligned}
$$

Now, by (i), $e^{\prime}>\operatorname{ord}(1-y)$ and by (vii), $e^{\prime}>\operatorname{ord}(w-1)$. Moreover, $\operatorname{ord}(w-1)$ $+\operatorname{ord}(y-1) \geq e^{\prime}+f$, with $f=\left(\frac{p-1}{p}\right)\left(i^{\prime}+k+e^{\prime}\right) \geq 0$ by (xi). Thus, Corollary 2.5 
yields

$$
\begin{aligned}
G\left(y^{\delta} \zeta_{1}^{\beta}, w^{e} \zeta_{1}^{d}\right) & \equiv G\left(y^{\delta}, w^{e} \zeta_{1}^{d}\right)\left(w^{e} \zeta_{1}^{d}\right)^{\beta} \\
& \equiv G\left(y^{\delta}, w^{e}\right) y^{d \delta} w^{e \beta} \zeta_{1}^{d \beta} \quad\left(\bmod \pi^{p f} R\right) \\
& =G(y, w)^{e \delta} y^{d \delta} w^{e \beta} \zeta_{1}^{d \beta}+\pi^{p f} m_{\delta, \beta, e, d},
\end{aligned}
$$

where $m_{\delta, \beta, e, d}$ is in $R$. So

$$
\Gamma=\zeta_{1}^{c \delta+e \alpha+d \beta}\left(1+\pi^{p f} n_{\delta, \beta, e, d}\right),
$$

for some element $n_{\delta, \beta, e, d}$ in $R$. Then

$$
\begin{aligned}
& \eta=\sum_{c, d, e, \alpha, \beta, \delta=0}^{q, r, s, \sigma, \tau, \varepsilon} C \Gamma \\
& =\sum_{c, d, e, \alpha, \beta, \delta=0}^{q, r, s, \sigma, \tau, \varepsilon} C\left(1+\pi^{p f} n_{\delta, \beta, e, d}\right) \zeta_{1}^{c \delta+e \alpha+d \beta} \\
& =\sum_{c, d, e, \alpha, \beta, \delta=0}^{q, r, s, \sigma, \tau, \varepsilon} C \zeta_{1}^{c \delta+e \alpha+d \beta} \\
& +\pi^{p f} \sum_{c, d, e, \alpha, \beta, \delta=0}^{q, r, s, \sigma, \tau, \varepsilon} C n_{\delta, \beta, e, d} \zeta_{1}^{c \delta+e \alpha+d \beta} .
\end{aligned}
$$

Now the first sum

$$
\sum_{c, d, e, \alpha, \beta, \delta=0}^{q, r, s, \sigma, \tau, \varepsilon} C \zeta_{1}^{c \delta+e \alpha+d \beta}=\sum_{c, \delta=0}^{q, \varepsilon} C(c, \delta) \zeta_{1}^{c \delta} \cdot \sum_{e, \alpha=0}^{s, \sigma} C(e, \alpha) \zeta_{1}^{e \alpha} \cdot \sum_{d, \beta=0}^{r, \tau} C(d, \beta) \zeta_{1}^{d \beta}
$$

and (cf. the proof of Theorem 1.2)

$$
\sum_{c, \delta=0}^{q, \varepsilon} C(c, \delta) \zeta_{1}^{c \delta}
$$

has order $\geq e^{\prime} q$ and $\geq e^{\prime} \varepsilon$, hence order $\geq q i+\varepsilon i^{\prime}$. Similarly for the others. In the second sum, let

$$
C(d, e, \beta, \delta) n_{\delta, \beta, e, d} \zeta_{1}^{d \beta}=h(\delta, \beta, e, d) .
$$

Then the second sum is

$$
\begin{aligned}
& \pi^{p f} \sum_{d, e, \beta, \delta=0}^{r, s, \tau, \varepsilon} h(\delta, \beta, e, d) \cdot \sum_{c=0}^{q}\left(\begin{array}{l}
q \\
c
\end{array}\right)(-1)^{q-c} \zeta_{1}^{\delta c} \cdot \sum_{\alpha=0}^{\sigma}\left(\begin{array}{l}
\sigma \\
\alpha
\end{array}\right)(-1)^{\sigma-\alpha} \zeta_{1}^{e \alpha} \\
& =\pi^{p f} \sum_{d, e, \beta, \delta=0}^{r, s, \tau, \varepsilon} h(\delta, \beta, e, d)\left(\zeta_{1}^{\delta}-1\right)^{q}\left(\zeta_{1}^{e}-1\right)^{\sigma}
\end{aligned}
$$

which has order $\geq p f+q e^{\prime}+\sigma e^{\prime}$. So we want

$$
p f+q e^{\prime}+\sigma e^{\prime} \geq q i+r j+s k+\sigma k^{\prime}+\tau j^{\prime}+\varepsilon i^{\prime} .
$$

The worst case is when $q=\sigma=0, r=\tau=\varepsilon=s=p-1$, in which case we have

$$
p f \geq(p-1)\left(i^{\prime}+k+e^{\prime}\right),
$$

which holds since $f=\left(\frac{p-1}{p}\right)\left(i^{\prime}+k+e^{\prime}\right)$. 
Pairs of triangular $R$-Hopf orders satisfying the conditions of Theorem 3.7 are called duality Hopf orders.

Suppose a Hopf order $H \subseteq K C_{p^{3}}$ induces the short exact sequences

$$
R \rightarrow A(i, j, u) \rightarrow H \rightarrow H(k) \rightarrow R
$$

and

$$
R \rightarrow A\left(k^{\prime}, j^{\prime}, z\right) \rightarrow H^{*} \rightarrow H\left(i^{\prime}\right) \rightarrow R .
$$

Then $H$ satisfies the "valuative condition for $n=3$ " [Un96, $\S 4.0$ ] if either $p k \leq l$ or $p i^{\prime} \leq \hat{l}$, where $H(i, l)=\mathcal{L}(A(i, j, u))$ and $H\left(k^{\prime}, \hat{l}\right)=\mathcal{L}\left(A\left(k^{\prime}, j^{\prime}, z\right)\right)$. If $H$ satisfies this condition, then at least one of the short exact sequences above can be written as the Baer product of a generically trivial extension and a distinguished extension of Hopf orders. Hence the structure of $H$ can be characterized. This generalizes Greither's method of Gr92 for Hopf orders of rank $p^{2}$.

Recall that the maximal Larson order in $A(i, j, u)$ is $H(i, l)$, where

$$
l=\left\{\begin{array}{lc}
j & \text { if } \operatorname{ord}(u-1) \geq i^{\prime}+j, \\
i-e^{\prime}+\operatorname{ord}(u-1) & \text { otherwise }
\end{array}\right.
$$

and the maximal Larson order in $A\left(k^{\prime}, j^{\prime}, z\right)$ is $H\left(k^{\prime}, \hat{l}\right)$, where

$$
\hat{l}= \begin{cases}j^{\prime} & \text { if } \operatorname{ord}(z-1) \geq k+j^{\prime}, \\ k^{\prime}-e^{\prime}+\operatorname{ord}(z-1) & \text { otherwise. }\end{cases}
$$

Theorem 3.8. A duality Hopf order $A(i, j, k, u, v, w)$ satisfies the valuative condition.

Proof. Since $e^{\prime}>\operatorname{ord}(\hat{u}-1)$, and $e^{\prime}>\operatorname{ord}(\hat{z}-1)$, then from (xi),

$$
e^{\prime}+\operatorname{ord}(\hat{z}-1)>e^{\prime}+\frac{p-1}{p}\left(i^{\prime}+k+e^{\prime}\right),
$$

hence

$$
\operatorname{ord}(\hat{z}-1)>\frac{e^{\prime}}{p}
$$

and likewise, $\operatorname{ord}(\hat{u}-1)>e^{\prime} / p$. Thus ord $(u-1)=\operatorname{ord}(z-1)=e^{\prime} / p$, which gives $p i^{\prime} \leq j^{\prime}$ and $p k \leq j$ by (v) and (vi).

By (xi) and Proposition 2.3, one has

$$
\operatorname{ord}(G(\hat{u}, \hat{z})-1) \geq \frac{p-1}{p}\left(i^{\prime}+k+e^{\prime}\right)>\frac{e^{\prime}}{p^{2}} .
$$

Now if $i^{\prime}+p k>e^{\prime} / p$, then by (ii) $\operatorname{ord}\left(v^{p} \zeta_{2}-1\right)>e^{\prime} / p$, thus since $v x \zeta_{3} G(\hat{u}, \hat{z})=1$, $\operatorname{ord}\left(x^{p}-1\right)>e^{\prime} / p$. So $e^{\prime} / p \geq k+p i^{\prime}$ by (viii), and the valuation condition $p i^{\prime} \leq \hat{l}$ holds.

If $e^{\prime} / p \geq i^{\prime}+p k$, then the valuation condition $p k \leq l$ holds.

Proposition 3.9. Let $A(i, j, k, u, v, w)$ be duality. Then either $A(i, j, u)$ or $A\left(k^{\prime}, j^{\prime}, z\right)$ is dual Larson. 
Proof. If $\operatorname{ord}(\hat{u}-1) \geq i^{\prime}+j$, then $A(i, j, u)$ is dual Larson, so suppose $i^{\prime}+j>$ $\operatorname{ord}(\hat{u}-1)$. Then by $(\mathrm{xi})$,

$$
\begin{aligned}
i^{\prime}+j+\operatorname{ord}(\hat{z}-1) & >e^{\prime}+\frac{p-1}{p}\left(i^{\prime}+k+e^{\prime}\right), \\
i^{\prime}+\operatorname{ord}(\hat{z}-1) & >j^{\prime}+\frac{(p-1) i^{\prime}}{p}+\frac{(p-1) k}{p}+\frac{e}{p}, \\
\operatorname{ord}(\hat{z}-1) & >j^{\prime}+\frac{e-i^{\prime}}{p}+\frac{(p-1) k}{p}, \\
\operatorname{ord}(\hat{z}-1) & >j^{\prime}+\frac{i+(p-1) k}{p}, \\
\operatorname{ord}(\hat{z}-1) & >j^{\prime}+k,
\end{aligned}
$$

since $i \geq k$. Thus $A\left(k^{\prime}, j^{\prime}, z\right)$ is dual Larson.

In view of the above proposition and Theorem 3.8, it is natural to compare duality Hopf orders to the cohomological Hopf orders of Theorem 1.7. There are duality Hopf orders which are not cohomological, as the following example shows.

Example 3.10. Let $p=3, e^{\prime}=306, e^{\prime} / p=102, i=272, j=204, k=6$, so that $i^{\prime}=34, j^{\prime}=102, k^{\prime}=300$. Let

- $\operatorname{ord}(\hat{u}-1)=237$, then

- $\operatorname{ord}(u-1)=102 ;$ let

- $\operatorname{ord}(\hat{z}-1)=305$, then

- $\operatorname{ord}(z-1)=102$ and

- $\operatorname{ord}(G(\hat{u}, \hat{z})-1)=237+305-306=236$. Choose $x$ with

- $\operatorname{ord}(x-1)=34$ and

- $\operatorname{ord}\left(x^{3} \zeta_{2}-1\right) \geq 111$. Define $v$ by $G(\hat{u}, \hat{z}) v x \zeta_{3}=1$, then

- $\operatorname{ord}(v-1)=37$ and

- $\operatorname{ord}\left(v^{3} \zeta_{2}-1\right)=102$.

Then one verifies that all of the valuation inequalities hold, to yield a pair of dual Hopf orders, as follows:

(i) $237=\operatorname{ord}(\hat{u}-1) \geq 216 \geq i^{\prime} / 3+j$;

(ii) $102=\operatorname{ord}\left(v^{3} \zeta_{2}-1\right)>i^{\prime}+3 k=52$;

(iii) $237=\operatorname{ord}(\hat{u}-1) \geq i^{\prime}=34$;

(iv) $543=\operatorname{ord}(\hat{u}-1)+\operatorname{ord}\left(z^{3}-1\right) \geq 3 k+i^{\prime}+e^{\prime}=358$;

(v) $102=\operatorname{ord}(z-1) \geq j^{\prime} / 3+k=40$;

(vi) $102=\operatorname{ord}(u-1) \geq i^{\prime}+j / 3=102$;

(vii) $305=\operatorname{ord}(\hat{z}-1) \geq j^{\prime}+k / 3=104$;

(viii) $\operatorname{ord}\left(x^{p} \zeta_{2}-1\right) \geq 111>108=k+3 i^{\prime}$;

(ix) $305=\operatorname{ord}(\hat{z}-1) \geq k=6$;

(x) $611=\operatorname{ord}(\hat{z}-1)+\operatorname{ord}\left(u^{p}-1\right) \geq 3 i^{\prime}+k+e^{\prime}=414$;

(xi) $542=\operatorname{ord}(\hat{z}-1)+\operatorname{ord}(\hat{u}-1) \geq 537 \geq e^{\prime}+\left(\frac{2}{3}\right)\left(k+i^{\prime}+e^{\prime}\right)$.

Now $A(i, j, u)=A(272,204, u)$ is not dual Larson, but $A\left(k^{\prime}, j^{\prime}, z\right)=A(300,102, z)$ is dual Larson, but not Larson. We have $\mathcal{L}(A(300,102, z))=H(300,96)$. However, $3 i^{\prime} \not \leq 96$ as required for $A\left(k^{\prime}, j^{\prime}, i^{\prime}, z, x, y\right)$ to be cohomological. 
Moreover, no cohomological Hopf order with $e^{\prime} / p>\operatorname{ord}(w-1)=\operatorname{ord}(\hat{z}-1)$ can be duality, for by (xi) and (i) of Theorem 3.7 $\operatorname{ord}(\hat{z}-1)$ must satisfy

$$
e^{\prime}+\operatorname{ord}(\hat{z}-1) \geq \operatorname{ord}(\hat{u}-1)+\operatorname{ord}(\hat{z}-1) \geq e^{\prime}+\left(\frac{p-1}{p}\right)\left(e^{\prime}+i^{\prime}+k\right) .
$$

Remark 3.11. Now that we have discussed triangular Hopf orders in some detail, a natural question arises: Is every Hopf order in $K C_{p^{3}}$ triangular? Intuitively, the answer would seem to be "no". Suppose $H$ is a rank $p^{3}$ Hopf order that is an extension of a rank $p^{2}$ Hopf order by $H(i)$. The classes of examples we have constructed require that the parameter $i$ be close to $e^{\prime}$. For example, if $H$ is a duality Hopf order, then inequalities (iii) and (vi) of Theorem 3.7 are

$$
\begin{gathered}
\text { (iii) } \operatorname{ord}(\hat{u}-1) \geq i^{\prime}, \\
\text { (vi) } \operatorname{ord}(u-1) \geq i^{\prime}+j / p .
\end{gathered}
$$

Since $\hat{u}=\left(u \zeta_{2}\right)^{-1}$, it follows from (iii) and (vi) that

$$
e^{\prime} / p \geq i^{\prime}
$$

and hence

$$
i \geq\left(\frac{p-1}{p}\right) e^{\prime}
$$

Similarly, if $H$ is a ILD Hopf order, then $i^{\prime} \leq e^{\prime} / p$. Thus a rank $p^{3}$ Hopf order that is an extension of a rank $p^{2}$ Hopf order by $H(i)$, where $i$ is sufficiently smaller than $e^{\prime}$, cannot be duality or ILD.

In fact, we essentially showed in CU03 that there are Hopf orders arising from formal groups with $i$ sufficiently smaller than $e^{\prime}$ that cannot be triangular. In the next section we review this result, and investigate the relationship between "formal group" Hopf orders and triangular Hopf orders in more detail.

\section{Formal group Hopf ORDERS}

In CU03, Theorem 2.1] the authors give a general construction of Hopf orders in $K C_{p^{n}}$ as the representing algebras of kernels of isogenies $f: \mathcal{F} \rightarrow \mathcal{G}$ of degree 2 dimension $n$ polynomial formal groups. These are the so-called formal group Hopf orders. We obtained the following theorem:

Theorem 4.1 (CU03, Theorem 2.1]). Suppose $\Theta$ is an $n \times n$ lower triangular matrix with entries in $R$ for which $\operatorname{det}(\Theta) \neq 0$ and $\operatorname{ord}\left(\theta_{r, r}\right)>0$ for all $r$. Suppose, for all $\ell$, that $\operatorname{ord}\left(\theta_{\ell, i}\right)<\operatorname{ord}\left(\theta_{\ell, \ell}\right)$ for all $i<\ell$ such that $\theta_{\ell, i} \neq 0$, and suppose also that there exists numbers $q$ and $d$ so that $\operatorname{ord}\left(\theta_{\ell, i}\right) \geq(1-q) \operatorname{ord}\left(\theta_{\ell, \ell}\right)$ and $\operatorname{ord}\left(\theta_{\ell, \ell}\right) \geq d \operatorname{ord}\left(\theta_{\ell+1, \ell+1}\right)$, where

$$
\begin{aligned}
0 & <q<\frac{p-1}{2 p-1} \\
\operatorname{ord}\left(\theta_{1,1}\right) & <\left(\frac{p-1}{p}\right)\left(\frac{d-1}{d-1+q}\right) e^{\prime}
\end{aligned}
$$

and

$$
d \geq \frac{p}{1-q}+\frac{q}{1-\frac{1-q}{p}}
$$

Then $\Theta$ gives rise to an $R$-Hopf order $H_{\Theta}$ in $K C_{p^{n}}$. 
Remark 4.2. The structure of $H_{\Theta}$ can be determined as follows. Let $U=\left(u_{i, j}\right)$ denote the lower triangular matrix which is the inverse of $\Theta$. Then, following CS98, p. 71],

$$
H_{\Theta}=R\left[z_{1}, z_{2}, \ldots, z_{n}\right]
$$

where

$$
\begin{aligned}
z_{1} & =u_{1,1}\left(g^{p^{n-1}}-1\right), \\
z_{2} & =u_{2,1}\left(g^{p^{n-1}}-1\right)+u_{2,2}\left(g^{p^{n-2}}-1\right), \\
& \vdots \\
z_{n} & =u_{n, 1}\left(g^{p^{n-1}}-1\right)+\ldots+u_{n, n}(g-1),
\end{aligned}
$$

and $\langle g\rangle=C_{p^{n}}$.

In this section we relate formal group Hopf orders when $n=3$ to the classes of triangular Hopf orders of the previous sections.

We begin by recalling a result from CU03. Suppose $q \in \mathbb{Q}$ satisfies

$$
0<q<\frac{p-1}{2 p-1}
$$

Put

$$
d=\frac{p}{1-q}+\frac{q}{1-\frac{1-q}{p}}>p .
$$

Let $i$ and $j$ be integers with $i>d j$ and

$$
i \leq\left(\frac{p-1}{p}\right)\left(\frac{d-1}{d-1+q}\right) e^{\prime} .
$$

Let $s$ and $k$ be integers with $j>d k, s<k$, and $s=(1-q) k$, that is, $q=1-\frac{s}{k}$. Set

$$
\Theta=\left(\begin{array}{ccc}
\pi^{i} & 0 & 0 \\
0 & \pi^{j} & 0 \\
0 & \pi^{s} & \pi^{k}
\end{array}\right)
$$

Then by Theorem 4.1, $\Theta$ yields an $R$-Hopf order $H_{\Theta}$ in $K C_{p^{3}}$ of the form

$$
H_{\Theta}=R\left[\frac{g^{p^{2}}-1}{\pi^{i}}, \frac{g^{p}-1}{\pi^{j}}, \frac{-\pi^{s}\left(g^{p}-1\right)}{\pi^{j+k}}+\frac{g-1}{\pi^{k}}\right] .
$$

In [CU03, Theorem 4.2] we proved that if $i<\left(1-\frac{1}{p}-\frac{1}{p^{2}}\right) e^{\prime}$, then $H_{\Theta}$ is not of the form

$$
R\left[\frac{g^{p^{2}}-1}{\pi^{i}}, \frac{g^{p}-1}{\pi^{j}}, \frac{b_{x, y} g-1}{\pi^{k}}\right] .
$$

A review of the proof of [CU03, Theorem 4.2] shows that with only obvious notational changes, that proof yields:

Theorem 4.3. If

$$
i<\left(1-\frac{1}{p}-\frac{1}{p^{2}}\right) e^{\prime}
$$

then the R-Hopf order $H_{\Theta}$ in $K C_{p^{3}}$ defined above is not triangular.

We can also show that not every triangular Hopf order is formal group. 
Theorem 4.4. Suppose $p>2$. Let $H=H(i, j, k, u, v, w)$ be an ILD Hopf order with $j^{\prime}+(k / p) \leq \operatorname{ord}(w-1)<j^{\prime}+(k / 2)$. Then $H$ cannot represent the kernel of any isogeny of formal groups $f: \mathcal{F} \rightarrow \mathcal{G}$.

Proof. $H$ induces the short exact sequence

$$
R \rightarrow H(i) \rightarrow H \rightarrow H(j, k, w) \rightarrow R,
$$

where $H(j, k, w)$ is a Greither order in $K C_{p^{2}}$. Suppose $H$ represents the kernel of an isogeny $f: \mathcal{F} \rightarrow \mathcal{G}$. Then the coalgebra structure of $H$ is induced by the 3 dimensional polynomial formal group $\mathcal{F}$. It follows that the coalgebra structure of the Greither order $H(j, k, w)$ is induced by a 2-dimensional polynomial formal group $\mathcal{F}^{\prime}$. But this contradicts [CU03, Theorem 3.0] since ord $(1-w)<j^{\prime}+(k / 2)$.

Confirming a statement in Remark 3.11, we have

Proposition 4.5. Suppose $H_{\Theta}$ is a formal group Hopf order of rank $p^{3}$, where $\operatorname{ord}\left(\theta_{1,1}\right)$ satisfies the inequality

$$
\operatorname{ord}\left(\theta_{1,1}\right)=i<\left(\frac{p-1}{p}\right)\left(\frac{d-1}{d-1+q}\right) e^{\prime}
$$

of Theorem 4.1. Then $H_{\Theta}$ is not an ILD Hopf order.

The proof is trivial: any ILD Hopf order satisfies $e^{\prime} / p \geq i^{\prime}$, hence $i \geq\left(\frac{p-1}{p}\right) e^{\prime}$.

Triangular and formal group Hopf orders are not mutually exclusive, however. Certainly any Larson order in $K C_{p^{3}}$ is both formal group and triangular. To see more precisely how formal group Hopf orders and their duals relate to triangular Hopf orders, we need to look more carefully at formal group Hopf orders when $n=3$. Theorem 4.1 gives sufficient conditions to construct Hopf orders for any $n$, but when $n=3$, it is not sharp. Here is a more precise result when $n=3$, one that allows $i$ to be close to $e^{\prime}$.

Theorem 4.6. Let

$$
\Theta=\left(\begin{array}{ccc}
\pi^{i} & 0 & 0 \\
b \pi^{r} & \pi^{j} & 0 \\
c \pi^{s} & d \pi^{t} & \pi^{k}
\end{array}\right)
$$

with $b, c, d$ units of $R$, where $r<j$ and $s, t<k$. Then the $R$-algebra $H_{\Theta}$ is a Hopf order in $K C_{p^{3}}$ if the following inequalities hold:

$$
\begin{aligned}
& e^{\prime}>i>p j, \\
& 2 r \geq j>r>p k, \\
& 2 t \geq k>t, \\
& 2 s \geq k>s \text {, } \\
& (p-1) i^{\prime}=(p-1)\left(e^{\prime}-i\right)>p(k-s), \\
& (p-1) i^{\prime}=(p-1)\left(e^{\prime}-i\right)>p(j-r)+p(k-t), \\
& i-p j>p(k-t) \text {, } \\
& 2 r-j \geq k-t \text {. }
\end{aligned}
$$

Proof. We begin with a brief review of the construction of formal group Hopf orders of [CU03] adapted to the $n=3$ case. Let $\bar{x}=\left(x_{1}, x_{2}, x_{3}\right)^{T}, \bar{y}=\left(y_{1}, y_{2}, y_{3}\right)^{T}$, and let $\mathbb{G}_{m}^{3}$ denote the 3 -dimensional multiplicative formal group. Let $\Theta$ be a $3 \times 3$ 
lower triangular matrix with entries $\theta_{i j}$. Under certain conditions on the entries of $\Theta$, there exist 3 -dimensional formal groups $\mathcal{F}$ and $\mathcal{F}^{(p)}$ defined by

$$
\begin{gathered}
\mathcal{F}(\bar{x}, \bar{y})=\Theta^{-1} \mathbb{G}_{m}^{3}(\Theta \bar{x}, \Theta \bar{y}), \\
\mathcal{F}^{(p)}(\bar{x}, \bar{y})=\left(\Theta^{(p)}\right)^{-1} \mathbb{G}_{m}^{3}\left(\Theta^{(p)} \bar{x}, \Theta^{(p)} \bar{y}\right),
\end{gathered}
$$

respectively, where $\Theta^{(p)}$ denotes the $3 \times 3$ matrix whose $i j$ th entry is $\theta_{i j}^{p}$. Let $[*]: \mathbb{G}_{m}^{3} \rightarrow \mathbb{G}_{m}^{3}$ denote the homomorphism of formal groups defined by

$$
[*](\bar{x})=\left(\left(1+x_{1}\right)^{p}-1,\left(1+x_{1}\right)^{-1}\left(1+x_{2}\right)^{p}-1,\left(1+x_{2}\right)^{-1}\left(1+x_{3}\right)^{p}-1\right)^{T} .
$$

Then one can impose additional restrictions on $\Theta$ so that the map

$$
f(\bar{x})=\left(\Theta^{(p)}\right)^{-1}[*](\Theta \bar{x})
$$

is an isogeny of formal groups $f: \mathcal{F} \rightarrow \mathcal{F}^{(p)}$. The representing algebra of the kernel of $f$ is a Hopf order in $K C_{p^{3}}$ of the form $H_{\Theta}$.

To construct $H_{\Theta}$ we first find conditions for the formal group $\mathcal{F}$ to be defined over $R$. Since $\left.\mathcal{F}(\bar{x}, \bar{y})=\Theta^{-1} \mathbb{G}_{m}^{3}(\Theta \bar{x}), \Theta \bar{y}\right)$, it is routine to verify that

$$
\begin{aligned}
\mathcal{F}_{1}=x_{1} & +y_{1}+\pi^{i} x_{1} y_{1}, \\
\mathcal{F}_{2}=x_{2} & +y_{2}+\left(\frac{-b \pi^{r+2 i}}{\pi^{i+j}}\right) x_{1} y_{1}+\left(\frac{b^{2} \pi^{2 r}}{\pi^{j}}\right) x_{1} y_{1}+b \pi^{r}\left(x_{1} y_{2}+x_{2} y_{1}\right)+\pi^{j} x_{2} y_{2}, \\
\mathcal{F}_{3}=x_{3} & +y_{3}+\left(\frac{\pi^{2 i}\left(b d \pi^{r+t}-c \pi^{s+j}\right)}{\pi^{i+j+k}}-\frac{d \pi^{t} b^{2} \pi^{2 r}}{\pi^{j+k}}+\frac{c^{2} \pi^{2 s}}{\pi^{k}}\right) x_{1} y_{1} \\
& +\left(\frac{-b d \pi^{t} \pi^{r+j}}{\pi^{j+k}}+\frac{c d \pi^{s+t}}{\pi^{k}}\right)\left(x_{1} y_{2}+x_{2} y_{1}\right)+\left(\frac{c \pi^{s+k}}{\pi^{k}}\right)\left(x_{1} y_{3}+x_{3} y_{1}\right) \\
& +\left(\frac{-d \pi^{t} \pi^{2 j}}{\pi^{j+k}}+\frac{d^{2} \pi^{2 t}}{\pi^{k}}\right) x_{2} y_{2}+\left(\frac{d \pi^{t+k}}{\pi^{k}}\right)\left(x_{2} y_{3}+x_{3} y_{2}\right)+\pi^{k} x_{3} y_{3} .
\end{aligned}
$$

So $\mathcal{F}$ is defined over $R$ if each of the above coefficients is in $R$, which is the case if the following inequalities hold:

$$
\begin{aligned}
r+i & \geq j, \\
2 r & \geq j, \\
s+i & \geq k, \\
i+r+t & \geq j+k, \\
t+2 r & \geq j+k, \\
t+r & \geq k, \\
t+j & \geq k, \\
2 t & \geq k, \\
s+t & \geq k, \\
2 s & \geq k .
\end{aligned}
$$

We also need $\mathcal{F}^{(p)}(\bar{x}, \bar{y})=\left(\Theta^{(p)}\right)^{-1} \mathbb{G}_{m}^{3}\left(\Theta^{(p)} \bar{x}, \Theta^{(p)} \bar{y}\right)$ to be defined over $R$, but the inequalities become the same. Now we seek conditions on $\Theta$ so that $f(\bar{x})$ is 
defined over $R$, where

$$
\begin{aligned}
f(\bar{x}) & =\left(\Theta^{(p)}\right)^{-1}[*](\Theta \bar{x}) \\
& =\left(\Theta^{(p)}\right)^{-1}\left(\begin{array}{l}
\eta_{1} \\
\eta_{2} \\
\eta_{3}
\end{array}\right),
\end{aligned}
$$

where

$$
\begin{aligned}
& \eta_{1}=\left(1+\theta_{1,1} x_{1}\right)^{p}-1, \\
& \eta_{2}=\frac{\left(1+\theta_{2,1} x_{1}+\theta_{2,2} x_{2}\right)^{p}-\left(1+\theta_{1,1} x_{1}\right)}{\left(1+\theta_{1,1} x_{1}\right)}, \\
& \eta_{3}=\frac{\left(1+\theta_{3,1} x_{1}+\theta_{3,2} x_{2}+\theta_{3,3} x_{3}\right)^{p}-\left(1+\theta_{2,1} x_{1}+\theta_{2,2} x_{2}\right)}{1+\theta_{2,1} x_{1}+\theta_{2,2} x_{2}} .
\end{aligned}
$$

Assuming that $\operatorname{ord}\left(\theta_{i, j}\right)<e^{\prime}$ for all $i, j$ such that $\theta_{i, j} \neq 0$, we have $[*](\Theta \bar{x})=$

$$
\left(\begin{array}{c}
\theta_{1,1}^{p} x_{1}^{p}+p \theta_{1,1} z_{1}^{\prime} \\
\left(\theta_{2,1}^{p} x_{1}^{p}+\theta_{2,2}^{p} x_{2}^{p}+p \theta_{2,1} z_{2}^{\prime}-\theta_{1,1} x_{1}\right)\left(1-\theta_{1,1} z_{3}^{\prime}\right) \\
\left(\theta_{3,1}^{p} x_{1}^{p}+\theta_{3,2}^{p} x_{2}^{p}+\theta_{3,3}^{p} x_{3}^{p}+p \theta_{3,1} z_{4}^{\prime}+p \theta_{3,2} z_{5}^{\prime}-\theta_{2,1} x_{1}-\theta_{2,2} x_{2}\right)\left(1+\theta_{2,1} \pi^{r} z_{6}^{\prime}\right)
\end{array}\right)
$$

with $z_{i}^{\prime}$ in $\left.R[\bar{x}]\right]$.

Inserting the entries of $\Theta$, we find that $f(x)=\bar{x}^{(p)}+w$, where $\bar{x}^{(p)}=\left(x_{1}^{p}, x_{2}^{p}, x_{3}^{p}\right)^{T}$ and $w=\left(w_{1}, w_{2}, w_{3}\right)^{T}$, where

$$
\begin{aligned}
w_{1} & =\frac{p \pi^{i}}{\pi^{p i}} z_{1}, \\
w_{2} & =\frac{\pi^{p r} p \pi^{i}}{\pi^{p i+p j}} z_{2}+\frac{p \pi^{r}}{\pi^{p j}} z_{3}+\frac{\pi^{i}}{\pi^{p j}} z_{4}, \\
w_{3} & =\frac{p \pi^{i+p s}}{\pi^{p i+p k}} z_{5}+\frac{p \pi^{i+p r+p t}}{\pi^{p i+p j+p k}} z_{6}+\frac{p \pi^{p t+r}}{\pi^{p j+p k}} z_{7}+\frac{\pi^{p t+i}}{\pi^{p j+p k}} z_{8} \\
& +\frac{p \pi^{s}}{\pi^{p k}} z_{9}+\frac{p \pi^{t}}{\pi^{p k}} z_{10}+\frac{\pi^{r}}{\pi^{p k}} z_{11}
\end{aligned}
$$

with $z_{1}, \ldots, z_{11}$ in $R[[\bar{x}]]$. Thus for $f(\bar{x})$ to be defined over $R$ with

$$
\left.f(\bar{x}) \equiv \bar{x}^{(p)} \quad(\bmod \pi R[\bar{x}]]\right)
$$

it suffices that the following inequalities hold:

$$
\begin{aligned}
e+i & >p i, \\
e+p r+i & >p i+p j, \\
e+r & >p j, \\
i & >p j, \\
e+i+p s & >p i+p k, \\
e+i+p r+p t & >p i+p j+p k, \\
e+p t+r & >p j+p k, \\
p t+i & >p j+p k, \\
e+s & >p k, \\
e+t & >p k, \\
r & >p k .
\end{aligned}
$$

Collecting the two sets of inequalities for $\mathcal{F}$ and $f$ yields the theorem. 
These inequalities immediately yield:

Corollary 4.7. If $H=H_{\Theta}$ is a formal group Hopf order arising from Theorem 4.1 or Theorem 4.6 and $p>2$ or $k>0$ or $i<e^{\prime}$ or $j<i / p$ or $j^{\prime}<k^{\prime} / p$, then $H^{*}$ cannot be a formal group Hopf order.

Proof. Considering the hypotheses of each theorem, the valuation parameters $i, j$ and $k$ of $H$ satisfy $i \geq p j$ and $j \geq p k$. The valuation parameters of $H^{*}$ are then $k^{\prime}, j^{\prime}$ and $i^{\prime}$, and for $H^{*}$ to be a formal group Hopf order they would have to satisfy $k^{\prime} \geq p j^{\prime}$ and $j^{\prime} \geq p i^{\prime}$. But then $j \leq i / p \leq e^{\prime} / p$ and $j^{\prime} \leq k^{\prime} / p \leq e^{\prime} / p$, hence $e^{\prime} \leq 2 e^{\prime} / p$, which means that $p=2$ and all the inequalities in the statement of the corollary are equalities.

To treat duals of formal group Hopf orders arising from Theorem 4.6, we will use the following general duality result.

Theorem 4.8. Let $K$ contain a primitive $p^{n}$ th root of unity $\zeta_{n}$, and let $G=\langle g\rangle$ be cyclic of order $p^{n}$ with character group $\hat{G}=\langle\gamma\rangle$. Let $H \subset K G$ be a Hopf order and let $H_{1}=H \cap K\left\langle g^{p^{n-1}}\right\rangle=H(i)$ with $0 \leq i<e^{\prime}$. Let $\bar{H}=$ image of $H$ modulo $\left\langle g^{p^{n-1}}\right\rangle$. Let $A=\bar{H}^{*} \subset K\left\langle\gamma^{p}\right\rangle$. Let

$$
J=A\left[\frac{u \gamma-1}{\pi^{i^{\prime}}}\right]
$$

with $u \in K\left\langle\gamma^{p}\right\rangle$. If $\langle J, H\rangle_{n} \subset R$, then $J=H^{*}$ is a Hopf order in $K \hat{G}$.

Proof. First, we show that $\alpha=\frac{u \gamma-1}{\pi^{i^{\prime}}}$ satisfies a monic polynomial of degree $p$ with coefficients in $A$.

We have

$$
\gamma^{p} u^{p}=\left(1+\alpha \pi^{i^{\prime}}\right)^{p} \in K\left[\left\langle\gamma^{p}\right\rangle\right]
$$

and so since $e=(p-1) e^{\prime} \geq(p-1) i^{\prime}$,

$$
\alpha^{p}+\sum_{r=1}^{p-1}\left(\begin{array}{l}
p \\
r
\end{array}\right) \alpha^{r} \pi^{(r-p) i^{\prime}}=\frac{u^{p} \gamma^{p}-1}{\pi^{p i^{\prime}}}
$$

is in $H^{*} \cap K\left\langle\gamma^{p}\right\rangle=A$. Thus $u$ is a unit of $A$.

Now if $A$ has an $R$-basis $\left\{a_{\nu}\right\}, \nu=1, \ldots, p^{n-1}$, then $J$ has an $R$-basis $\left\{a_{\nu} \alpha^{k}\right\}$ with $\nu=1, \ldots, p^{n-1}, k=0, \ldots, p-1$, a basis with which we can compute the discriminant of $J$. The discriminant of $H^{*}$ may be obtained by dualizing the exact sequence of Hopf orders,

$$
R \rightarrow H(i)=H_{1} \rightarrow H \rightarrow \bar{H} \rightarrow R .
$$

We obtain

$$
R \rightarrow A \rightarrow H^{*} \rightarrow H_{1}^{*} \rightarrow R
$$

from which we have (from [Gr92]; cf. [C00, (22.18)])

$$
\operatorname{disc}\left(H^{*}\right)=\operatorname{disc}(A)^{p} \operatorname{disc}\left(H_{1}^{*}\right)^{p^{n-1}}=\operatorname{disc}(A)^{p} \pi^{p^{n-1} p(p-1) i} R,
$$

since $H_{1}^{*}=H\left(i^{\prime}\right)$ and $\operatorname{disc}\left(H\left(i^{\prime}\right)\right)=\pi^{p(p-1) i} R$ by [C00, (21.1)].

Now $J_{0}=A[u \gamma-1]=A[\gamma]$ is a Hopf order in $K \hat{G}$, and we can compute the discriminant of $A[\gamma]$ using the exact sequence:

$$
R \rightarrow A \rightarrow J_{0} \rightarrow H(0) \rightarrow R,
$$


namely,

$$
\operatorname{disc}\left(J_{0}\right)=\operatorname{disc}(A)^{p} \operatorname{disc}(H(0))^{p^{n-1}}=\operatorname{disc}(A)^{p} \pi^{p^{n-1} p(p-1) e^{\prime}} R .
$$

The matrix that multiplies the basis $\left\{a_{\nu} \alpha^{k}\right\}$ of $J$ to the basis $\left\{a_{\nu}(u \gamma-1)^{k}\right\}$ of $J_{0}$ is the matrix

$$
M=\operatorname{diag}\left(1, \ldots, 1, \pi^{i^{\prime}}, \ldots, \pi^{i^{\prime}}, \ldots, \pi^{(p-1) i^{\prime}}, \ldots, \pi^{(p-1) i^{\prime}}\right),
$$

whose determinant is $\pi^{i^{\prime}\left(p^{n-1} p(p-1)\right) / 2}$. So

$$
\operatorname{disc}\left(J_{0}\right)=\operatorname{det}(M)^{2} \operatorname{disc}(J)=\pi^{p^{n-1} p(p-1) i^{\prime}} \operatorname{disc}(J),
$$

and hence

$$
\begin{aligned}
\operatorname{disc}(J) & =\operatorname{disc}\left(J_{0}\right) \pi^{-p^{n-1} p(p-1) i^{\prime}} R \\
& =\operatorname{disc}(A)^{p} \pi^{p^{n-1} p(p-1) e^{\prime}-p^{n-1} p(p-1) i^{\prime}} R \\
& =\operatorname{disc}(A)^{p} \pi^{p^{n-1} p(p-1) i} R=\operatorname{disc}\left(H^{*}\right) .
\end{aligned}
$$

Since $J \subset H^{*}$ and have equal discriminants, $J=H^{*}$.

We now show that the dual of a formal group Hopf order of rank $p^{3}$ is triangular. Let

$$
\Theta=\left(\begin{array}{ccc}
\pi^{i} & 0 & 0 \\
b \pi^{r} & \pi^{j} & 0 \\
c \pi^{s} & d \pi^{t} & \pi^{k}
\end{array}\right)
$$

with $b, c, d$ units of $R$, satisfying Theorem 4.6, and let $U=\left(u_{i, j}\right)$ be the inverse of $\Theta$. Then $H_{\Theta}=R\left[z_{1}, z_{2}, z_{3}\right]$, where

$$
\begin{aligned}
& z_{1}=u_{1,1}\left(g^{p^{2}}-1\right), \\
& z_{2}=u_{2,1}\left(g^{p^{2}}-1\right)+u_{2,2}\left(g^{p}-1\right), \\
& z_{3}=u_{3,1}\left(g^{p^{2}}-1\right)+u_{3,2}\left(g^{p}-1\right)+u_{3,3}(g-1) .
\end{aligned}
$$

Let

$$
J=R\left[\frac{\gamma^{p^{2}}-1}{\pi^{k^{\prime}}}, \frac{a_{z} \gamma^{p}-1}{\pi^{j^{\prime}}}, \frac{a_{x} b_{y} \gamma-1}{\pi^{i^{\prime}}}\right]
$$

with $z$ satisfying

$$
u_{3,2}\left(\zeta_{1}-1\right)+u_{3,3}\left(\zeta_{2} z-1\right)=0,
$$

with $y$ satisfying

$$
u_{2,1}\left(\zeta_{1}-1\right)+u_{2,2}\left(\zeta_{2} y-1\right)=0
$$

and with $x$ satisfying

$$
u_{3,1}\left(\zeta_{1}-1\right)+u_{3,2}\left(\zeta_{2} y-1\right)+u_{3,3}\left(\zeta_{3} x-1\right)=0 .
$$

Then one sees easily that

$$
\begin{gathered}
z=\zeta_{2}^{-1}\left(1+\pi^{t-j} d\left(\zeta_{1}-1\right)\right), \\
y=\zeta_{2}^{-1}\left(1+\pi^{r-i} b\left(\zeta_{1}-1\right)\right)
\end{gathered}
$$

and

$$
x=\zeta_{3}^{-1}\left(1+\pi^{s-i} c\left(\zeta_{1}-1\right)\right) .
$$

Theorem 4.9. Suppose $\Theta$ is as above so that $\mathcal{F}_{\Theta}$ and the isogeny $f_{\Theta}$ are defined over $R$. Then the dual of $H_{\Theta}$ is triangular. 
Proof. We show that $H_{\Theta}^{*}=J$, defined above. Using Theorem 4.8 it suffices to show that

$$
\left\langle J, H_{\Theta}\right\rangle \subset R
$$

We know that

$$
J_{0}=R\left[\frac{\gamma^{p^{2}}-1}{\pi^{k^{\prime}}}, \frac{a_{z} \gamma^{p}-1}{\pi^{j^{\prime}}}\right]
$$

is the dual of $H_{\Theta_{2}}$, the image of $H_{\Theta}$ under the map sending $g^{p^{2}}$ to 1 , where

$$
\Theta_{2}=\left(\begin{array}{cc}
\pi^{j} & 0 \\
d \pi^{t} & \pi^{k}
\end{array}\right)
$$

So since $H_{\Theta}$ is a Hopf order and its dual contains $J_{0}$, it suffices to show that $\frac{a_{x} b_{y} \gamma-1}{\pi^{i^{\prime}}}$ is in $H_{\Theta}^{*}$. So we need to show that for all $0 \leq l, m, n \leq p-1$,

$$
\left\langle a_{x} b_{y} \gamma-1, z_{1}^{l} z_{2}^{m} z_{3}^{n}\right\rangle \in \pi^{i^{\prime}} R .
$$

Since

for $l+m+n>0$ and

$$
\left\langle 1, z_{1}^{l} z_{2}^{m} z_{3}^{n}\right\rangle=0
$$

it suffices to show that

$$
\left\langle a_{x} b_{y} \gamma-1,1\right\rangle=0
$$

$$
\left\langle a_{x} b_{y} \gamma, z_{1}^{l} z_{2}^{m} z_{3}^{n}\right\rangle \in \pi^{i^{\prime}} R
$$

for $l+m+n>0$. Now we have

$$
\left\langle a_{x} b_{y} \gamma, g^{p^{2} r+p s+t}\right\rangle=\zeta_{1}^{r}\left(y \zeta_{2}\right)^{s}\left(x \zeta_{3}\right)^{t}
$$

for $0 \leq s, t \leq p-1$ and any $r \geq 0$. So for $0<m+n \leq p-1$, we have

$$
\begin{aligned}
\left\langle a_{x} b_{y} \gamma, z_{1}^{l} z_{2}^{m} z_{3}^{n}\right\rangle & =\left(u_{1,1}\left(\zeta_{1}-1\right)\right)^{l}\left(u_{2,1}\left(\zeta_{1}-1\right)+u_{2,2}\left(y \zeta_{2}-1\right)\right)^{m} \\
& \quad \cdot\left(u_{3,1}\left(\zeta_{1}-1\right)+u_{3,2}\left(y \zeta_{2}-1\right)+u_{3,3}\left(x \zeta_{3}-1\right)\right)^{n} \\
& =0,
\end{aligned}
$$

by the way we defined $x$ and $y$.

Since

$$
u_{2,1}\left(\zeta_{1}-1\right)+u_{2,2}\left(y \zeta_{2}-1\right)=0
$$

we have

$$
\begin{aligned}
z_{2} & =u_{2,1}\left(g^{p^{2}}-1\right)+u_{2,2}\left(g^{p}-1\right) \\
& =u_{2,1}\left(g^{p^{2}}-\zeta_{1}\right)+u_{2,2}\left(g^{p}-y \zeta_{2}\right)
\end{aligned}
$$

and since

$$
u_{3,1}\left(\zeta_{1}-1\right)+u_{3,2}\left(y \zeta_{2}-1\right)+u_{3,3}\left(x \zeta_{3}-1\right)=0
$$

we have

$$
\begin{aligned}
z_{3} & =u_{3,1}\left(g^{p^{2}}-1\right)+u_{3,2}\left(g^{p}-1\right)+u_{3,3}(g-1) \\
& =u_{3,1}\left(g^{p^{2}}-\zeta_{1}\right)+u_{3,2}\left(g^{p}-y \zeta_{2}\right)+u_{3,3}\left(g-x \zeta_{3}\right) .
\end{aligned}
$$

Set $b_{1}=g^{p^{2}}-\zeta_{1}, b_{2}=g^{p}-y \zeta_{2}, b_{3}=g-x \zeta_{3}$. If we set $\lambda=\zeta_{1}-1$, then we have

$$
z_{1}=u_{1,1}\left(b_{1}+\lambda\right),
$$

and

$$
z_{1}^{l} z_{2}^{m} z_{3}^{n}=u_{1,1}^{l}\left(b_{1}+\lambda\right)^{l}\left(u_{2,1} b_{1}+u_{2,2} b_{2}\right)^{m}\left(u_{3,1} b_{1}+u_{3,2} b_{2}+u_{3,3} b_{3}\right)^{n}
$$


is an $R$-linear combination of terms of the form

$$
B=u_{1,1}^{l} \lambda^{l_{2}} u_{2,1}^{m_{1}} u_{2,2}^{m_{2}} u_{3,1}^{n_{1}} u_{3,2}^{n_{2}} u_{3,3}^{n_{3}} b_{1}^{l_{1}+m_{1}+n_{1}} b_{2}^{m_{2}+n_{2}} b_{3}^{n_{3}}
$$

with $l_{1}+l_{2}=l, m_{1}+m_{2}=m<p$, and $n_{1}+n_{2}+n_{3}=n<p$.

Set

$$
\phi=\left\langle a_{x} b_{y} \gamma,-\right\rangle: K G \rightarrow K
$$

Then $\phi$ maps $R G$ to $R$ and $\phi(B)=0$ if $l_{1}+m_{1}+n_{1}>0$ or $n_{3}>0$. Thus $\phi\left(z_{1}^{l} z_{2}^{m} z_{3}^{n}\right)$ is a linear combination of terms of the form

$$
u_{1,1}^{l} \lambda^{l} u_{2,2}^{m} u_{3,2}^{n} \phi\left(b_{2}^{m+n}\right) .
$$

Terms of this form equal 0 if $0<m+n<p$. If $m+n=p+t$, then

$$
\phi\left(b_{2}^{p+t}\right)=p \phi(\xi)+\phi\left(\left(g^{p^{2}}-y^{p} \zeta_{1}\right)\left(g^{p}-\zeta_{2} y\right)^{t}\right)
$$

with $\xi$ in $R G$. The second term in the right side equals 0 if $t>0$, and $=\zeta_{1}\left(1-y^{p}\right)$ if $m+n=p$. Thus, with $m+n=p+t, \phi\left(z_{1}^{l} z_{2}^{m} z_{3}^{n}\right)$ is a linear combination of terms of the form

$$
\left(u_{1,1} \lambda\right)^{l} u_{2,2}^{m} u_{3,2}^{n} p \phi(\xi)+\left(u_{1,1} \lambda\right)^{l} u_{2,2}^{m} u_{3,2}^{n}\left(\zeta_{1}\left(1-y^{p}\right)\right)
$$

with $\phi(\xi) \in R$ and the second term occurring only for $m+n=p$.

Note that

$$
\begin{aligned}
\operatorname{ord}\left(u_{2,2}\right) & =-j, \\
\operatorname{ord}\left(u_{3,2}\right) & =t-j-k, \\
\operatorname{ord}\left(u_{1,1}\right) & =-i, \\
\operatorname{ord}\left(1-y^{p}\right) & \geq \min \left\{e^{\prime}, p i^{\prime}+p r\right\} .
\end{aligned}
$$

Thus

follows if

$$
\operatorname{ord}\left(\left(u_{1,1} \lambda\right)^{l} u_{2,2}^{m} u_{3,2}^{n} p\right) \geq i^{\prime}
$$

$$
l\left(e^{\prime}-i\right)-m j-n(t-j-k)+e>i^{\prime}
$$

and for that to hold ( $\operatorname{since} \operatorname{ord}\left(u_{1,1} \lambda\right)>0$ ) it suffices that

$$
e>i^{\prime}+(p-1) j+(p-1)(j+(k-t)) .
$$

But $i>p j+p(k-t)$ from Theorem 4.6, so

$$
i^{\prime}+(p-1) j+\left(\frac{p-1}{p}\right) i \geq i^{\prime}+(p-1) j+(p-1)(j+(k-t)) .
$$

Now $\left(\frac{2(p-1)}{p}\right) i+i^{\prime}>i^{\prime}+(p-1) j+\left(\frac{p-1}{p}\right) i$ since $i>p j$, and $e \geq\left(\frac{2(p-1)}{p}\right) i+i^{\prime}$ since $e^{\prime} \geq i$, thus the required inequality holds.

Also, for $m+n=p$,

$$
\operatorname{ord}\left(\left(u_{1,1} \lambda\right)^{l} u_{2,2}^{m} u_{3,2}^{n} \zeta_{1}\left(1-y^{p}\right)\right) \geq i^{\prime}
$$

if

$$
\begin{aligned}
\min \left\{e^{\prime}, p i^{\prime}+p r\right\}+m(-j)+n(-j-k+t) \\
\geq \min \left\{e^{\prime}, p i^{\prime}+p r\right\}-p j+(p-1)(t-k) \geq i^{\prime}
\end{aligned}
$$

or

$$
\min \left\{e^{\prime}, p i^{\prime}+p r\right\}+i-p j-(p-1)(k-t) \geq e^{\prime}
$$

But from Theorem 4.6, we have

$$
i-p j-(p-1)(k-t)>0
$$


so the desired inequality follows if $\min \left\{e^{\prime}, p i^{\prime}+p r\right\} \geq e^{\prime}$. To show

$$
p i^{\prime}+p r-p j-(p-1)(k-t) \geq i^{\prime}
$$

it suffices to show that

$$
(p-1) i^{\prime} \geq p(j-r)+(p-1)(k-t)
$$

which follows from the inequality from Theorem 4.6:

$$
(p-1) i^{\prime} \geq p(j-r)+p(k-t) .
$$

This completes the proof.

We seek conditions so that $H_{\Theta}$ itself is triangular.

Theorem 4.10. Suppose $\Theta$ is as above so that $\mathcal{F}_{\Theta}$ and the isogeny $f_{\Theta}$ are defined over $R$. Then $J=H_{\Theta}^{*}$ is ILD if $e^{\prime} / p>i^{\prime}+r$ and $e^{\prime} / p \geq k+p i^{\prime}$.

Proof. From Theorem 4.9 we have the triangular Hopf order

$$
H_{\Theta}^{*}=J=R\left[\frac{\gamma^{p^{2}}-1}{\pi^{k^{\prime}}}, \frac{a_{z} \gamma^{p}-1}{\pi^{j^{\prime}}}, \frac{a_{x} b_{y} \gamma-1}{\pi^{i^{\prime}}}\right]
$$

with

$$
\begin{gathered}
z=\zeta_{2}^{-1}\left(1+\pi^{t-j} d\left(\zeta_{1}-1\right)\right), \\
y=\zeta_{2}^{-1}\left(1+\pi^{r-i} b\left(\zeta_{1}-1\right)\right)
\end{gathered}
$$

and

$$
x=\zeta_{3}^{-1}\left(1+\pi^{s-i} c\left(\zeta_{1}-1\right)\right) .
$$

Note $e^{\prime} / p \geq k$ by Theorem 4.6, so the Hopf order $\mathcal{L} \mathcal{D}\left(A\left(k^{\prime}, j^{\prime}, z\right)\right)$ exists. Since $\operatorname{ord}(\hat{z}-1)=t+j^{\prime}<k+j^{\prime}$,

$$
\mathcal{L D}\left(A\left(k^{\prime}, j^{\prime}, z\right)\right)=H\left(\varrho^{\prime}, k\right)^{*}=H\left(k^{\prime}, \varrho, \zeta_{2}^{-1}\right),
$$

where $\varrho$ satisfies $\operatorname{ord}(\hat{z}-1)=k+\varrho$. Thus $\varrho^{\prime}=k+j-t$.

Let $l$ satisfy

$$
\left\{\begin{array}{l}
l=\varrho \text { if } e^{\prime} / p \geq k+\varrho \\
e^{\prime} / p=k+l \text { if } e^{\prime} / p<k+\varrho .
\end{array}\right.
$$

We need $p i^{\prime} \leq l$. If $e^{\prime} / p<k+\varrho$, this follows from the assumption $e^{\prime} / p \geq k+p i^{\prime}$. But by a condition of Theorem 4.6. we have $(p-1) e^{\prime}>p(j-t)$, which yields $k+\varrho>e^{\prime} / p$, and so $p i^{\prime} \leq l$. Thus $J$ is ILD if the inequalities (A)-(D) hold, where

$$
\begin{gathered}
\operatorname{ord}\left(1-x^{p}\right) \geq k+p i^{\prime}, \\
\operatorname{ord}(1-y) \geq(k+j-t) / p+i^{\prime}, \\
\operatorname{ord}(1-y) \geq k+j-t+\left(i^{\prime} / p\right), \\
\operatorname{ord}\left(1-x^{p} y^{-1}\right) \geq p k+i^{\prime} .
\end{gathered}
$$

(A) We have

$$
\operatorname{ord}\left(1-x^{p}\right) \geq \min \left\{e^{\prime} / p, p i^{\prime}+p s, e+i^{\prime}+s\right\}
$$

thus (A) holds given that $e^{\prime} / p \geq p i^{\prime}+k, p s \geq k$, and $e+i^{\prime}+s \geq p i^{\prime}+k$.

(B) Since $e^{\prime} / p>i^{\prime}+r, \operatorname{ord}(1-y)=i^{\prime}+r$. Now

$$
i^{\prime}+r \geq(k+j-t) / p+i^{\prime}
$$

follows from $t+2 r \geq j+k$, a condition of Theorem 4.6 
(C) The condition of Theorem 4.6. $(p-1) i^{\prime} \geq p(k-t)+p(j-r)$ implies (C).

(D) We have

$$
\operatorname{ord}\left(1-y^{-1} x^{p}\right) \geq \min \left\{e+i^{\prime}+s, i^{\prime}+r, p i^{\prime}+p s\right\},
$$

which implies (D) by Theorem 4.6 .

We conclude this section by finding sufficient conditions on a formal group Hopf order $H$ to be triangular. Since $H^{*}$ is triangular by Theorem 4.9, if we find conditions on $H^{*}$ to be duality, then $H$ will be triangular.

Proposition 4.11. Let $J=A\left(k^{\prime}, j^{\prime}, i^{\prime}, z, x, y\right)$ be the dual of a formal group Hopf order $H_{\Theta}$ with $\Theta$ as in Theorem 4.6. If $\operatorname{ord}(\hat{y}-1)=i^{\prime}+r=e^{\prime} / p$ and $\operatorname{ord}(y-1)$ satisfies $e^{\prime}>\operatorname{ord}(1-y)$ and

$$
\operatorname{ord}(\hat{z}-1)+\operatorname{ord}(y-1)=t+j^{\prime}+\operatorname{ord}(y-1) \geq e^{\prime}+\left(\frac{p-1}{p}\right)\left(e^{\prime}+i^{\prime}+k\right),
$$

then $H_{\Theta}$ is triangular.

Proof. We have

$$
\begin{aligned}
\operatorname{ord}(\hat{z}-1) & =t+j^{\prime}, \\
\operatorname{ord}(\hat{y}-1) & =r+i^{\prime}, \\
\operatorname{ord}\left(x \zeta_{3}-1\right) & =s+i^{\prime},
\end{aligned}
$$

where $i, j, k, r, s, t$ satisfy the inequalities of Theorem 4.6. Following Theorem 3.7 . $H=A(i, j, k, u, v, w)$ is free of rank $p$ over $A(i, j, u)$ if (Proposition 3.5)

(i) $\operatorname{ord}(u-1) \geq i^{\prime}+j / p$,

(ii) $\operatorname{ord}(\hat{u}-1) \geq i^{\prime} / p+j$,

(iii) $\operatorname{ord}\left(v^{p} \zeta_{2}-1\right) \geq i^{\prime}+p k$,

(iv) $\operatorname{ord}(\hat{u}-1) \geq i^{\prime}$,

(v) $\operatorname{ord}\left(w \zeta_{2}-1\right) \geq j^{\prime} / p+k$,

(vi) $\operatorname{ord}(\hat{u}-1)+\operatorname{ord}\left(w^{p} \zeta_{1}-1\right) \geq e^{\prime}+i^{\prime}+p k$,

and $H=J^{*}$ if $w=\hat{z}, y=\hat{u}, v x \zeta_{3} G(\hat{u}, \hat{z})=1$ and

(xi) $\operatorname{ord}(\hat{z}-1)+\operatorname{ord}(\hat{u}-1) \geq e^{\prime}+\left(\frac{p-1}{p}\right)\left(e^{\prime}+i^{\prime}+k\right)$.

To begin with condition (xi), $\operatorname{ord}(\hat{z}-1)=j^{\prime}+t$, so ord $(\hat{u}-1)=\operatorname{ord}(y-1)$ must satisfy

$$
\operatorname{ord}(\hat{u}-1)=\operatorname{ord}(y-1) \geq e^{\prime}+\left(\frac{p-1}{p}\right)\left(e^{\prime}+i^{\prime}+k\right)-\left(j^{\prime}+t\right) .
$$

Now $\operatorname{ord}(\hat{y}-1)=i^{\prime}+r$. From Remark 1.4, if $\operatorname{ord}(\hat{y}-1) \neq e^{\prime} / p$, then $\operatorname{ord}(y-1) \leq$ $e^{\prime} / p$; but

$$
e^{\prime} / p \geq e^{\prime}+\left(\frac{p-1}{p}\right)\left(e^{\prime}+i^{\prime}+k\right)-\left(j^{\prime}+t\right)
$$

is impossible for $p \geq 3$, for

$$
j^{\prime}+t+e^{\prime} / p<e^{\prime}+e^{\prime} / p+e^{\prime} / p^{2}<e^{\prime}+\left(\frac{p-1}{p}\right) e^{\prime}<e^{\prime}+\left(\frac{p-1}{p}\right)\left(e^{\prime}+i^{\prime}+k\right) .
$$

Thus we must have $\operatorname{ord}(\hat{y}-1)=i^{\prime}+r=e^{\prime} / p$ and $\operatorname{ord}(y-1)$ so large (but still $<e^{\prime}$ ) that (xi) holds. 
We check (i)-(vi). Since ord $(y-1)=\operatorname{ord}(\hat{u}-1)$ satisfies (xi), one sees easily from the inequalities of Theorem 4.6 that (ii), (iv) and (vi) hold. As for the others:

(i): $\operatorname{ord}(u-1)=\operatorname{ord}(\hat{y}-1)=e^{\prime} / p=i^{\prime}+r \geq i^{\prime}+j / p$ since $j \leq 2 r$. from

(v): If $\operatorname{ord}\left(w \zeta_{2}-1\right)=\operatorname{ord}(z-1)=j^{\prime}+t<e^{\prime} / p$, then $j^{\prime}+t \geq j^{\prime} / p+k$ follows

$$
(p-1) j^{\prime} \geq(p-1) i^{\prime} \geq p(j-r)+p(k-t) \geq p(k-t) .
$$

If $\operatorname{ord}\left(w \zeta_{2}-1\right)=\operatorname{ord}(z-1) \geq e^{\prime} / p$, then (v) follows from $e^{\prime}=j^{\prime}+j>j^{\prime}+p k$.

(iii): To show $\operatorname{ord}\left(v^{p} \zeta_{2}-1\right) \geq i^{\prime}+p k$, we observe that $e^{\prime}>\operatorname{ord}(\hat{z}-1)$ and $e^{\prime}>\operatorname{ord}(1-y)$, hence by Proposition 2.3,

$$
\begin{aligned}
\operatorname{ord}(G(\hat{u}, \hat{z})-1) & =\operatorname{ord}(G(y, w)-1) \\
& =\operatorname{ord}(y-1)+\operatorname{ord}(w-1)-e^{\prime} \\
& \geq\left(\frac{p-1}{p}\right)\left(e^{\prime}+i^{\prime}+k\right)
\end{aligned}
$$

by the assumption for (xi). We also have $\operatorname{ord}\left(x \zeta_{3}-1\right)=i^{\prime}+s$, and so, since $v x \zeta_{3} G(\hat{u}, \hat{z})=1$, we have

$$
\operatorname{ord}\left(v^{p} \zeta_{2}-1\right) \geq \min \left\{p i^{\prime}+p s, e^{\prime} / p\right\}
$$

Now $(p-1) i^{\prime} \geq p(k-s)$, so $p i^{\prime}+p s \geq i^{\prime}+p k$. Also, $e^{\prime} / p=i^{\prime}+r>i^{\prime}+p k$. Thus all the inequalities hold, and so $H=J^{*}$ is a triangular Hopf order.

\section{ReAlizability AND Hopf ORDERS}

For the moment we assume that $H$ is an $R$-Hopf order in $K G$ where $G$ is an abelian group.

An $H$-Galois algebra is a finitely generated projective $R$-algebra $A$ together with an $H$-module algebra structure $\beta: H \otimes_{R} A \rightarrow A$ for which the map

$$
H \otimes A \rightarrow \operatorname{End}_{R}(A),
$$

defined by $h \otimes x \mapsto(y \mapsto x \cdot \beta(h \otimes y))$, is bijective. The most interesting $H$ Galois algebras that one encounters are those which occur as rings of integers $S$ of abelian extensions $L / K$ with group $G$. In the terminology of C. Greither Gr92, an $R$-Hopf order $H$ in $K G$ is realizable as a Galois group, or more simply, realizable if there exists a Galois extension $L / K$ with group $G$ for which $S$ is an $H$-Galois algebra. If $H$ is realizable via the extension $L / K$, then the module algebra map $\beta: H \otimes_{R} S \rightarrow S$ becomes just the classical Galois action $K G \otimes L \rightarrow L$ upon tensoring with $K$.

Greither Gr92, Theorem II.3.2] has shown that $A(i, j, u), j>0$, is realizable if and only if $p$ divides $j$ and $\operatorname{ord}(u-1)=i^{\prime}+(j / p)$.

Quite generally, N. Byott By04 shows that a Hopf order $H$ in $K C_{p^{n}}$ with local dual $H^{*}$ is realizable iff $H^{*}$ is monogenic as an $R$-algebra.

Byott's result applies to the realizable Hopf orders of Greither. Thus the linear dual of the Greither order $A(i, j, u)$ with $j>0$ and $p j \leq i$ must be monogenic.

Theorem 5.1. Let $H=A(i, j, u)$ be an $R$-Hopf order in $K C_{p^{2}}$ with $\operatorname{ord}(u-1)=$ $i^{\prime}+(j / p)$. Then $H^{*}=A\left(j^{\prime}, i^{\prime}, \hat{u}\right)$ is monogenic with generator $\frac{a_{\hat{u}} \gamma-1}{\pi^{i^{\prime}}}$. 
Proof. From section 1 we know that the linear dual of $H=A(i, j, u)$ is the $R$-Hopf order $H^{*}=R\left[\frac{\gamma^{p}-1}{\pi^{j^{\prime}}}, \frac{a_{\hat{u}} \gamma-1}{\pi^{i^{\prime}}}\right]$. We claim that

$$
R\left[\frac{a_{\hat{u}} \gamma-1}{\pi^{i^{\prime}}}\right]=H^{*}
$$

Certainly $R\left[\frac{a_{\hat{u}} \gamma-1}{\pi^{i^{\prime}}}\right] \subseteq H^{*}$ so it suffices to show that the generators of $H^{*}$ are in $R\left[\frac{a_{\hat{u}} \gamma-1}{\pi^{i^{\prime}}}\right]$. Evidently, we only need to show that

$$
\frac{\gamma^{p}-1}{\pi^{j^{\prime}}} \in R\left[\frac{a_{\hat{u}} \gamma-1}{\pi^{i^{\prime}}}\right]
$$

Put $\alpha=\frac{a_{\hat{u}} \gamma-1}{\pi^{i^{\prime}}}$. Then

$$
\alpha^{p}+\sum_{r=0}^{p-1}\left(\begin{array}{l}
p \\
r
\end{array}\right) \alpha^{r} \pi^{(p-r) i^{\prime}}=\frac{a_{\hat{u}}^{p} \gamma^{p}-1}{\pi^{p i^{\prime}}} .
$$

Since $e^{\prime} \geq i^{\prime}$, the left-hand side of the above equation is in $R\left[\frac{a_{\hat{\imath}} \gamma-1}{\pi^{i^{\prime}}}\right]$, hence,

$$
R\left[\frac{a_{\hat{u}}^{p} \gamma^{p}-1}{\pi^{p i^{\prime}}}\right] \subseteq R\left[\frac{a_{\hat{u}} \gamma-1}{\pi^{i^{\prime}}}\right]
$$

Now observe that

$$
\frac{a_{\hat{u}}^{p} \gamma^{p}-1}{\pi^{p i^{\prime}}}=\frac{a_{\hat{u}^{p} \zeta_{1}}-1}{\pi^{p i^{\prime}}}=\frac{a_{u-p}-1}{\pi^{p i^{\prime}}}
$$

with $\operatorname{ord}\left(u^{-p}-1\right)=\operatorname{ord}\left(u^{p}-1\right)=p i^{\prime}+j=p i^{\prime}+\left(j^{\prime}\right)^{\prime}$, by hypothesis, hence

$$
R\left[\frac{a_{\hat{u}}^{p} \gamma^{p}-1}{\pi^{p i^{\prime}}}\right]=R\left[\frac{a_{u^{-p}}-1}{\pi^{p i^{\prime}}}\right]=H\left(j^{\prime}\right),
$$

by Proposition 2.1. Therefore

$$
H\left(j^{\prime}\right) \subseteq R\left[\frac{a_{\hat{u}} \gamma-1}{\pi^{i^{\prime}}}\right],
$$

and thus $\frac{\gamma^{p}-1}{\pi^{j^{\prime}}} \in R\left[\frac{a_{\hat{u}} \gamma-1}{\pi^{i^{\prime}}}\right]$. It follows that

$$
H^{*}=R\left[\frac{a_{\hat{u}} \gamma-1}{\pi^{i^{\prime}}}\right] .
$$

We now discuss the realizability of the Hopf orders in $K C_{p^{3}}$ given in this paper.

Theorem 5.2. An ILD Hopf order that is not cohomological is not realizable.

Proof. Let $H=H(i, j, k, u, v, w)$ be an ILD Hopf order with ord $(\hat{u}-1)<i^{\prime}+j$, that is, $H$ is an ILD Hopf order which is not cohomological. If $H$ is realizable, then so is $\bar{H}=A(j, k, w)$, the image of $H$ under the mapping $g^{p^{2}} \mapsto 1$. Thus $\operatorname{ord}(w-1)=j^{\prime}+k / p<\varrho^{\prime}+k / p$, which contradicts condition (C) of Proposition 1.8 .

Theorem 5.3. No duality Hopf order is realizable. 
Proof. Suppose $H=H(i, j, k, u, v, w)$ is a realizable duality Hopf order. Then $\operatorname{ord}(u-1)=i^{\prime}+j / p$ and $\operatorname{ord}(w-1)=j^{\prime}+k / p$ by [Gr92, Lemma II.1.6]. Then $\operatorname{ord}\left(u^{p}-1\right)=p i^{\prime}+j$ since $i^{\prime}+j / p<i^{\prime}+i=e^{\prime}$, and $p i^{\prime}+j+j^{\prime}+k / p=p i^{\prime}+e^{\prime}+k / p$. But inequality (x) of Theorem 3.7 requires

$$
\operatorname{ord}\left(u^{p}-1\right)+\operatorname{ord}(w-1) \geq p i^{\prime}+e^{\prime}+k .
$$

This implies $k / p \geq k$, which is impossible.

Theorem 5.4. If $p>2$, no formal group Hopf order $H_{\Theta}$ is realizable.

Proof. Let $\bar{H}=A(j, k, w)$ denote the image of $H_{\Theta}$ under the mapping $g^{p^{2}} \mapsto 1$. Then the coalgebra structure of $\bar{H}$ is given by a 2-dimensional generically split polynomial formal group. Thus by [CU03, Theorem 3.0], ord $(w-1) \geq j^{\prime}+(k / 2)$. Now if $H_{\Theta}$ is realizable, then so is $A(j, k, w)$, which is impossible.

So we look at cohomological Hopf orders for realizability.

Theorem 5.5. Let $H=H\left(i, j, k, \zeta_{2}^{-1}, v, w\right), k>0$, be a cohomological Hopf order. If $H$ is realizable, then $p i^{\prime}=j^{\prime}$.

Proof. By [Gr92, Lemma II.1.6] we have ord $\left(\zeta_{2}-1\right)=e^{\prime} / p=i^{\prime}+j / p$ which yields $p i^{\prime}=j^{\prime}$.

So to find realizable cohomological Hopf orders we are restricted to the case $p i^{\prime}=j^{\prime}$. This subclass simplifies to the form

$$
H\left(p i^{\prime}, i^{\prime}\right)^{*}\left[\frac{a_{v} b_{v^{p}} g-1}{\pi^{k}}\right]=R\left[\frac{g^{p^{2}}-1}{\pi^{i}}, \frac{a_{\zeta_{2}^{-1}} g^{p}-1}{\pi^{\left(p i^{\prime}\right)^{\prime}}}, \frac{a_{v} b_{v^{p}} g-1}{\pi^{k}}\right],
$$

with $v \in U_{i^{\prime}+\left(k / p^{2}\right)} \cap U_{\left(i^{\prime} / p\right)+k}, k>0$ (cf. [CU03, Remark 4.1].

Underwood [Un98, Theorem 3.2.0], [Un03, Theorem 3.3.1] has shown that these cohomological Hopf orders are realizable if and only if $p^{2}$ divides $k$ and $\operatorname{ord}(v-1)=$ $i^{\prime}+\left(k / p^{2}\right)$.

Hence by Byott's theorem, such Hopf orders must have monogenic duals.

Theorem 5.6. Let $H=H\left(p i^{\prime}, i^{\prime}\right)^{*}\left[\frac{a_{v} b_{v} p g-1}{\pi^{k}}\right]$ be a cohomological Hopf order with $k>0, \operatorname{ord}(v-1)=i^{\prime}+\left(k / p^{2}\right)$, where $p^{2}$ divides $k$. Then

$$
H^{*}=R\left[\frac{\gamma^{p^{2}}-1}{\pi^{k^{\prime}}}, \frac{a_{\hat{v}^{p}} \gamma^{p}-1}{\pi^{p i^{\prime}}}, \frac{a_{\hat{\hat{v}}} \gamma-1}{\pi^{i^{\prime}}}\right],
$$

where $\hat{v^{p}}=\left(v^{p} \zeta_{2}\right)^{-1}$, and $\hat{\hat{v}}=\left(v \zeta_{3}\right)^{-1}$, and $H^{*}$ is monogenic with generator $\frac{a_{\hat{v}} \gamma-1}{\pi^{i^{\prime}}}$.

Proof. We first show that the dual $H^{*}$ is of the claimed form.

By Theorem 4.8, we only need to show that $\langle H, J\rangle \subseteq R$, where

$$
J=R\left[\frac{\gamma^{p^{2}}-1}{\pi^{k^{\prime}}}, \frac{a_{\hat{v}^{p}} \gamma^{p}-1}{\pi^{p i^{\prime}}}, \frac{a_{\hat{\hat{v}}} \gamma-1}{\pi^{i^{\prime}}}\right],
$$


and for this it suffices to show that

$$
\operatorname{ord}\left(\left\langle\left(g^{p^{2}}-1\right)^{q}\left(a_{\zeta_{2}^{-1}} g^{p}-1\right)^{r}\left(a_{v} b_{v^{p}} g-1\right)^{s}, a_{x} \gamma-1\right\rangle_{3}\right) \geq q i+r\left(p i^{\prime}\right)^{\prime}+s k+i^{\prime},
$$

for $q, r, s=0, \ldots, p-1$, where $x=\hat{\hat{v}}$. Put

$$
\eta=\left\langle\left(g^{p^{2}}-1\right)^{q}\left(a_{\zeta_{2}^{-1}} g^{p}-1\right)^{r}\left(a_{v} b_{v^{p}} g-1\right)^{s}, a_{x} \gamma-1\right\rangle_{3} .
$$

Then

$$
\begin{aligned}
\eta & =\sum_{c, d, e, \delta=0}^{q, r, s, 1} C(c, d, e, \delta)\left\langle g^{p^{2} c} a_{\zeta_{2}^{-d}} g^{p d} a_{v^{e}} b_{\left(v^{p}\right)^{e}} g^{e}, a_{x^{\delta}} \gamma^{\delta}\right\rangle_{3} \\
& =\sum_{c, d, e, \delta=0}^{q, r, s, 1} C(c, d, e, \delta)\left\langle g^{p^{2} c+p d+e} a_{\zeta_{2}^{-d} v^{e}} b_{\left(v^{p}\right)^{e}}, \gamma^{\delta} a_{x^{\delta}}\right\rangle_{3} \\
& =\sum_{c, d, e, \delta=0}^{q, r, s, 1} C(c, d, e, \delta) \Gamma,
\end{aligned}
$$

where

$$
C(c, d, e, \delta)=\left(\begin{array}{l}
q \\
c
\end{array}\right)\left(\begin{array}{l}
r \\
d
\end{array}\right)\left(\begin{array}{l}
s \\
e
\end{array}\right)\left(\begin{array}{l}
1 \\
\delta
\end{array}\right)(-1)^{q-c}(-1)^{r-d}(-1)^{s-e}(-1)^{1-\delta}
$$

and

$$
\Gamma=\sum_{p a+b, p m+n} \zeta_{2}^{-d b} v^{e b}\left(v^{p}\right)^{a e} x^{\delta n}\left\langle e_{p a+b} g^{p^{2} c+p d+e}, \hat{e}_{p m+n} \gamma^{\delta}\right\rangle_{3} .
$$

By Lemma 3.4.

$$
\left\langle e_{p a+b} g^{p^{2} c+p d+e}, \hat{e}_{p m+n} \gamma^{\delta}\right\rangle_{3}=\frac{1}{p} \zeta_{1}^{-a(m-d)} \zeta_{3}^{\delta\left(p^{2} c+p d+e\right)} \delta_{e, n} \delta_{b, \delta}
$$

so since $v x \zeta_{3}=1$ we have

$$
\begin{aligned}
\Gamma & =\frac{1}{p} \sum_{a, m=0}^{p-1} \zeta_{2}^{-d \delta} v^{e \delta}\left(v^{p}\right)^{a e} x^{\delta e} \zeta_{1}^{-a(m-d)} \zeta_{3}^{\delta\left(p^{2} c+p d+e\right)} \\
& =\zeta_{1}^{c \delta}\left(\frac{1}{p} \sum_{a, m=0}^{p-1} \zeta_{1}^{-a(m-d)}\left(v^{p e}\right)^{a}\right) \\
& =\zeta_{1}^{c \delta}\left(\frac{1}{p} \sum_{a, m=0}^{p-1} \zeta_{1}^{-a m}\left(v^{p e} \zeta_{1}^{d}\right)^{a}\right) \\
& =\zeta_{1}^{c \delta} .
\end{aligned}
$$

So it suffices that

$$
\eta=\sum_{c, d, e, \delta=0}^{q, r, s, 1} C(c, d, e, \delta) \zeta_{1}^{c \delta} \in \pi^{q i+r\left(p i^{\prime}\right)^{\prime}+s k+i^{\prime}} R .
$$

Since this last sum is equal to

$$
\left(\sum_{d, e=0}^{r, s}\left(\begin{array}{l}
r \\
d
\end{array}\right)\left(\begin{array}{l}
s \\
e
\end{array}\right)(-1)^{r-d}(-1)^{s-e}\right)\left(\sum_{c, \delta=0}^{q, 1} C(c, \delta) \zeta_{1}^{c \delta}\right),
$$


and the first factor is 1 if $r, s=0$, and 0 otherwise, it suffices to show that

$$
\sum_{c, \delta=0}^{q, 1} C(c, \delta) \zeta_{1}^{c \delta} \in \pi^{q i+i^{\prime}} R
$$

for $q=0, \ldots, p-1$, which clearly holds. Thus by Theorem 4.8 , one has

$$
H^{*}=R\left[\frac{\gamma^{p^{2}}-1}{\pi^{k^{\prime}}}, \frac{a_{\hat{v}^{p}} \gamma^{p}-1}{\pi^{p i^{\prime}}}, \frac{a_{\hat{\hat{v}}} \gamma-1}{\pi^{i^{\prime}}}\right] .
$$

We claim that $H^{*}=R\left[\frac{a_{\hat{\hat{n}}} \gamma-1}{\pi^{i^{\prime}}}\right]$. Certainly

$$
R\left[\frac{a_{\hat{v}} \gamma-1}{\pi^{i^{\prime}}}\right] \subseteq H^{*}
$$

so it suffices to show that

$$
H^{*} \subseteq R\left[\frac{a_{\hat{\hat{v}}} \gamma-1}{\pi^{i^{\prime}}}\right],
$$

and for this it suffices to show that each generator of $H^{*}$ is in $R\left[\frac{a_{\hat{\hat{n}}} \gamma-1}{\pi^{i^{\prime}}}\right]$.

Since $e^{\prime} \geq p i^{\prime}, R\left[\frac{a_{\hat{v} p} \gamma^{p}-1}{\pi^{p i^{\prime}}}\right] \subseteq R\left[\frac{a_{\hat{v}} \gamma-1}{\pi^{i^{\prime}}}\right]$, so it suffices to show that

$$
R\left[\frac{a_{\hat{\hat{v}}^{p}} \gamma^{p}-1}{\pi^{p i^{\prime}}}\right]=R\left[\frac{\gamma^{p^{2}}-1}{\pi^{k^{\prime}}}, \frac{a_{\hat{v}^{p}} \gamma^{p}-1}{\pi^{p i^{\prime}}}\right],
$$

which follows if we can show that

$$
\operatorname{disc}\left(R\left[\frac{a_{\hat{\hat{v}}^{p}} \gamma^{p}-1}{\pi^{p i^{\prime}}}\right]\right)=\operatorname{disc}\left(R\left[\frac{\gamma^{p^{2}}-1}{\pi^{k^{\prime}}}, \frac{a_{\hat{\hat{v}^{p}}} \gamma^{p}-1}{\pi^{p i^{\prime}}}\right]\right) .
$$

We show that the discriminants above are equal by extending the discriminant argument of Proposition 2.1. We have

$$
\operatorname{disc}\left(R\left[\frac{\gamma^{p^{2}}-1}{\pi^{k^{\prime}}}, \frac{a_{\hat{v}^{p}} \gamma^{p}-1}{\pi^{p i^{\prime}}}\right]\right)=\left(\frac{p^{2}}{(p-1)\left(k^{\prime}+p i^{\prime}\right)}\right)^{p^{2}},
$$

by [Gr92, Lemma I.1.3a] and [La76]. Hence

$$
\begin{aligned}
\operatorname{ord}\left(\operatorname{disc}\left(R\left[\frac{\gamma^{p^{2}}-1}{\pi^{k^{\prime}}}, \frac{a_{\hat{v^{p}}} \gamma^{p}-1}{\pi^{p i^{\prime}}}\right]\right)\right) & =p^{2}\left(2 e-(p-1)\left(k^{\prime}+p i^{\prime}\right)\right) \\
& =p^{2}(p-1)\left(k+\left(p i^{\prime}\right)^{\prime}\right) .
\end{aligned}
$$

On the other hand,

$$
\begin{aligned}
\operatorname{disc} & \left(R\left[\frac{a_{\hat{\hat{v}}^{p}} \gamma^{p}-1}{\pi^{p i^{\prime}}}\right]\right) \\
& =\frac{1}{\pi^{p^{2}\left(p^{2}-1\right) p i^{\prime}}} \operatorname{disc}\left(1, a_{\hat{\hat{v}}^{p}} \gamma^{p}-1,\left(a_{\hat{\hat{v}}^{p}} \gamma^{p}-1\right)^{2}, \ldots,\left(a_{\hat{\hat{v}}^{p}} \gamma^{p}-1\right)^{p^{2}-1}\right) \\
& =\frac{1}{\pi^{p^{2}\left(p^{2}-1\right) p i^{\prime}}} \operatorname{disc}\left(1, a_{\hat{\hat{v}}^{p}} \gamma^{p},\left(a_{\hat{\hat{v}}^{p}} \gamma^{p}\right)^{2}, \ldots,\left(a_{\hat{\hat{v}}^{p}} \gamma^{p}\right)^{p^{2}-1}\right) \\
& =\frac{1}{\pi^{p^{2}\left(p^{2}-1\right) p i^{\prime}}} \operatorname{disc}\left(1, a_{\hat{\hat{v}}^{p} \zeta_{2}} b_{\zeta_{1}},\left(a_{\hat{\hat{v}}^{p} \zeta_{2}} b_{\zeta_{1}}\right)^{2}, \ldots,\left(a_{\hat{\hat{v}}^{p} \zeta_{2}} b_{\zeta_{1}}\right)^{p^{2}-1}\right) \\
& =\frac{1}{\pi^{p^{2}\left(p^{2}-1\right) p i^{\prime}}} \operatorname{disc}\left(1, a_{v^{-p}} b_{\zeta_{1}},\left(a_{v^{-p}} b_{\zeta_{1}}\right)^{2}, \ldots,\left(a_{v^{-p}} b_{\zeta_{1}}\right)^{p^{2}-1}\right) .
\end{aligned}
$$


Now

$$
\left(a_{v^{-p}} b_{\zeta_{1}}\right)^{k}=\sum_{p m+n=0}^{p^{2}-1}\left(v^{-p n} \zeta_{1}^{m}\right)^{k} e_{p m+n}
$$

for $0 \leq k \leq p^{2}-1$.

So

$$
\left(\begin{array}{c}
1 \\
a_{\hat{\hat{v}}^{p}} \gamma^{p} \\
\left(a_{\hat{v}^{p}} \gamma^{p}\right)^{2} \\
\vdots \\
\left(a_{\hat{\hat{v}}^{p}} \gamma^{p}\right)^{p^{2}-1}
\end{array}\right)=M\left(\begin{array}{c}
e_{0} \\
e_{1} \\
e_{2} \\
\vdots \\
e_{p^{2}-1}
\end{array}\right)
$$

where $M$ is the $p^{2} \times p^{2}$ matrix whose $(p m+n+1)$ st column, $0 \leq m, n \leq p-1$, is

$$
\left(\begin{array}{c}
1 \\
v^{-p n} \zeta_{1}^{m} \\
\left(v^{-p n} \zeta_{1}^{m}\right)^{2} \\
\left(v^{-p n} \zeta_{1}^{m}\right)^{3} \\
\vdots \\
\left(v^{-p n} \zeta_{1}^{m}\right)^{p^{2}-1}
\end{array}\right)
$$

Since

$$
\operatorname{disc}\left(e_{0}, e_{1}, \ldots, e_{p^{2}-1}\right)=R
$$

it suffices to compute $(\operatorname{det}(M))^{2}$. Since $M$ is Vandermonde,

$$
\operatorname{det}(M)=\prod_{0 \leq p m+n<p m^{\prime}+n^{\prime} \leq p^{2}-1}\left(v^{-p n^{\prime}} \zeta_{1}^{m^{\prime}}-v^{-p n} \zeta_{1}^{m}\right) .
$$

But

$$
\operatorname{ord}\left(v^{-p n^{\prime}} \zeta_{1}^{m^{\prime}}-v^{-p n} \zeta_{1}^{m}\right)=\operatorname{ord}\left(\zeta_{1}-1\right)=e^{\prime}
$$

if $n=n^{\prime}$, and

$$
\operatorname{ord}\left(v^{-p n^{\prime}} \zeta_{1}^{m^{\prime}}-v^{-p n} \zeta_{1}^{m}\right)=\operatorname{ord}\left(v^{p}-1\right)=p i^{\prime}+(k / p),
$$

for all other cases, thus,

$$
\operatorname{ord}(\operatorname{det}(M))=\left(\frac{p^{2}(p-1)}{2}\right) e^{\prime}+\left(\frac{p^{2}\left(p^{2}-1\right)}{2}-\frac{p^{2}(p-1)}{2}\right)\left(p i^{\prime}+(k / p)\right),
$$

and so

$$
\begin{aligned}
\operatorname{ord}\left(\operatorname{disc}\left(R\left[\frac{a_{\hat{v}^{p}} \gamma^{p}-1}{\pi^{p i^{\prime}}}\right]\right)\right)= & p^{2}(p-1) e^{\prime}+\left(p^{2}\left(p^{2}-1\right)\right. \\
& \left.-p^{2}(p-1)\right)\left(p i^{\prime}+(k / p)\right)-p^{2}\left(p^{2}-1\right) p i^{\prime} \\
= & p^{2}(p-1)\left(p i^{\prime}\right)^{\prime}+p^{2}(p-1) k \\
= & \operatorname{ord}\left(\operatorname{disc}\left(R\left[\frac{\gamma^{p^{2}}-1}{\pi^{k^{\prime}}}, \frac{a_{\hat{v}^{p}} \gamma^{p}-1}{\pi^{p i^{\prime}}}\right]\right)\right),
\end{aligned}
$$

which completes the proof. 
We seek other Hopf orders in $K C_{p^{3}}$ which are realizable. From Byott's theorem, we need only construct a Hopf order $H$ which is monogenic; then $H^{*}$ will be realizable.

Let $A\left(p^{2} k, k, v\right)=R\left[\frac{g^{p^{2}}-1}{\pi^{p^{2} k}}, \frac{a_{v} g^{p}-1}{\pi^{k}}\right]$ be a Greither order, $\left\langle g^{p}\right\rangle=C_{p^{2}}$, with $\operatorname{ord}\left(v^{p}-1\right)>e^{\prime} / p$, and set

$$
A=R\left[\frac{g^{p^{2}}-1}{\pi^{p^{2} k}}, \frac{g^{p}-1}{\pi^{p k}}, \frac{a_{v} g-1}{\pi^{k}}\right] .
$$

Theorem 5.7. The R-algebra $A$ as above is a monogenic Hopf order with realizable linear dual

$$
A^{*}=R\left[\frac{\gamma^{p^{2}}-1}{\pi^{k^{\prime}}}, \frac{a_{\zeta_{2}^{-1}} \gamma^{p}-1}{\pi^{(p k)^{\prime}}}, \frac{a_{\left(v \zeta_{3}\right)^{-1}} b_{\zeta_{2}^{-1}} \gamma-1}{\pi^{\left(p^{2} k\right)^{\prime}}}\right] .
$$

Proof. We first show that $A$ is a Hopf order in $K C_{p^{3}}$ using Proposition 1.1. We need $\frac{a_{v}^{p} g^{p}-1}{\pi^{p k}} \in H\left(p^{2} k, p k\right)$. To this end, write

$$
\begin{aligned}
\frac{a_{v}^{p} g^{p}-1}{\pi^{p k}} & =\frac{a_{v}^{p} g^{p}-a_{v}^{p} g^{p^{2}}+a_{v}^{p} g^{p^{2}}-1}{\pi^{p k}} \\
& =\frac{a_{v}^{p} g^{p}\left(1-g^{p^{2}-p}\right)}{\pi^{p k}}+\frac{a_{v}^{p} g^{p^{2}}-1}{\pi^{p k}} .
\end{aligned}
$$

Now $\frac{a_{v}^{p} g^{p}\left(1-g^{p^{2}-p}\right)}{\pi^{p k}} \in H\left(p^{2} k, p k\right)$ and $\frac{a_{v}^{p} g^{p^{2}}-1}{\pi^{p k}} \in H\left(p^{2} k\right)$ since $A\left(p^{2} k, k, v\right)$ is free over $H\left(p^{2} k\right)$ with basis $\left\{\left(\frac{a_{v} g^{p}-1}{\pi^{k}}\right)^{j}\right\}$. Now since $a_{v}$ is a unit in $A\left(p^{2} k, k, v\right)$, it suffices to show that

$$
\Delta\left(a_{v}\right) \equiv a_{v} \otimes a_{v} \quad\left(\bmod \pi^{k}\left(A\left(p^{2} k, k, v\right) \otimes A\left(p^{2} k, k, v\right)\right)\right) .
$$

But this follows from [C00, (31.10)] since ord $\left(v^{p}-1\right) \geq p\left(p^{2} k\right)^{\prime}+k$ by Theorem 1.5. Thus $A$ is an Hopf order.

We claim that $A$ is monogenic, generated by $\frac{a_{v} g-1}{\pi^{k}}$. Clearly $R\left[\frac{a_{v} g-1}{\pi^{k}}\right] \subseteq A$. So it remains to show that $A \subseteq R\left[\frac{a_{v} g-1}{\pi^{k}}\right]$. Since $e^{\prime} \geq k, R\left[\frac{a_{v}^{p} g^{p}-1}{\pi^{p k}}\right] \subseteq R\left[\frac{a_{v} g-1}{\pi^{k}}\right]$, so it suffices to show that $R\left[\frac{a_{v}^{p} g^{p}-1}{\pi^{p k}}\right]=R\left[\frac{g^{p^{2}}-1}{\pi^{p^{2} k}}, \frac{g^{p}-1}{\pi^{p k}}\right]$. Since $A$ is Hopf, $R\left[\frac{a_{v}^{p} g^{p}-1}{\pi^{p k}}\right] \subseteq$ $R\left[\frac{g^{p^{2}}-1}{\pi^{p^{2} k}}, \frac{g^{p}-1}{\pi^{p k}}\right]$.

$$
\operatorname{disc}\left(R\left[\frac{g^{p^{2}}-1}{\pi^{p^{2} k}}, \frac{g^{p}-1}{\pi^{p k}}\right]\right)=\left(\frac{p^{2}}{(p-1)\left(p^{2} k+p k\right)}\right)^{p^{2}}
$$

Hence

$$
\begin{aligned}
\operatorname{ord}\left(\operatorname{disc}\left(R\left[\frac{g^{p^{2}}-1}{\pi^{p^{2} k}}, \frac{g^{p}-1}{\pi^{p k}}\right]\right)\right) \\
\quad=p^{2}\left(2 e-(p-1)\left(p^{2} k+p k\right)\right)=p^{2}(p-1)\left(\left(p^{2} k\right)^{\prime}+(p k)^{\prime}\right) .
\end{aligned}
$$


On the other hand,

$$
\begin{aligned}
\operatorname{ord}\left(\operatorname{disc}\left(R\left[\frac{a_{v}^{p} g^{p}-1}{\pi^{p k}}\right]\right)\right) & =p^{2}(p-1)\left(e^{\prime}+p \operatorname{ord}\left(v^{p} \zeta_{2}-1\right)\right)-p^{2}\left(p^{2}-1\right) p k \\
& =p^{2}(p-1)\left(e^{\prime}+p \operatorname{ord}\left(v^{p} \zeta_{2}-1\right)-(p+1) p k\right) \\
& =p^{2}(p-1)\left(2 e^{\prime}-(p+1) p k\right) \\
& =p^{2}(p-1)\left(\left(p^{2} k\right)^{\prime}+(p k)^{\prime}\right),
\end{aligned}
$$

since $\operatorname{ord}\left(v^{p}-1\right)>e^{\prime} / p$. Thus $A$ is monogenic.

We next compute $A^{*}$. Put

$$
J=R\left[\frac{\gamma^{p^{2}}-1}{\pi^{k^{\prime}}}, \frac{a_{\zeta_{2}^{-1}} \gamma^{p}-1}{\pi^{(p k)^{\prime}}}, \frac{a_{\left(v \zeta_{3}\right)^{-1}} b_{\zeta_{2}^{-1}} \gamma-1}{\pi^{\left(p^{2} k\right)^{\prime}}}\right] .
$$

By Theorem 4.8, we only need to show that $\langle A, J\rangle \subseteq R$, and for this it suffices to show that

$$
\operatorname{ord}\left(\left\langle\left(g^{p^{2}}-1\right)^{q}\left(g^{p}-1\right)^{r}\left(a_{v} g-1\right)^{s}, a_{x} b_{y} \gamma-1\right\rangle_{3}\right) \geq q p^{2} k+r p k+s k+\left(p^{2} k\right)^{\prime},
$$

for $q, r, s=0, \ldots, p-1$, where $x=\hat{\hat{v}}, y=\zeta_{2}^{-1}$. Put

$$
\eta=\left\langle\left(g^{p^{2}}-1\right)^{q}\left(g^{p}-1\right)^{r}\left(a_{v} g-1\right)^{s}, a_{x} b_{y} \gamma-1\right\rangle_{3} .
$$

Then

$$
\begin{aligned}
\eta & =\sum_{c, d, e, \delta=0}^{q, r, s, 1} C(c, d, e, \delta)\left\langle g^{p^{2} c} g^{p d} a_{v^{e}} g^{e}, a_{x^{\delta}} b_{y^{\delta}} \gamma^{\delta}\right\rangle_{3} \\
& =\sum_{c, d, e, \delta=0}^{q, r, s, 1} C(c, d, e, \delta)\left\langle g^{p^{2} c+p d+e} a_{v^{e}}, \gamma^{\delta} a_{x^{\delta}} b_{y^{\delta}}\right\rangle_{3} \\
& =\sum_{c, d, e, \delta=0}^{q, r, s, 1} C(c, d, e, \delta) \Gamma,
\end{aligned}
$$

where

$$
C(c, d, e, \delta)=\left(\begin{array}{l}
q \\
c
\end{array}\right)\left(\begin{array}{l}
r \\
d
\end{array}\right)\left(\begin{array}{l}
s \\
e
\end{array}\right)\left(\begin{array}{l}
1 \\
\delta
\end{array}\right)(-1)^{q-c}(-1)^{r-d}(-1)^{s-e}(-1)^{1-\delta}
$$

and

$$
\Gamma=\sum_{p a+b, p m+n} v^{e b} x^{\delta n} y^{m \delta}\left\langle e_{p a+b} g^{p^{2} c+p d+e}, \hat{e}_{p m+n} \gamma^{\delta}\right\rangle_{3}
$$

By Lemma 3.4

$$
\left\langle e_{p a+b} g^{p^{2} c+p d+e}, \hat{e}_{p m+n} \gamma^{\delta}\right\rangle_{3}=\frac{1}{p} \zeta_{1}^{-a(m-d)} \zeta_{3}^{\delta\left(p^{2} c+p d+e\right)} \delta_{e, n} \delta_{b, \delta}
$$


so since $y \zeta_{2}=1, v x \zeta_{3}=1$ we have

$$
\begin{aligned}
\Gamma & =\frac{1}{p} \sum_{a, m=0}^{p-1} v^{e \delta} x^{\delta e} y^{m \delta} \zeta_{1}^{-a(m-d)} \zeta_{3}^{\delta\left(p^{2} c+p d+e\right)} \\
& =\frac{1}{p} \sum_{a, m=0}^{p-1} \zeta_{2}^{-m \delta} \zeta_{1}^{-a m} \zeta_{1}^{a d} \zeta_{1}^{c \delta} \zeta_{2}^{d \delta} \\
& =\zeta_{1}^{c \delta} \zeta_{2}^{d \delta} \frac{1}{p} \sum_{a, m=0}^{p-1} \zeta_{1}^{-a m}\left(\zeta_{2}^{-\delta}\right)^{m}\left(\zeta_{1}^{d}\right)^{a} \\
& =\zeta_{1}^{c \delta} \zeta_{2}^{d \delta} G\left(\zeta_{2}^{-\delta}, \zeta_{1}^{d}\right) \\
& =\zeta_{1}^{c \delta} .
\end{aligned}
$$

So it suffices that

$$
\eta=\sum_{c, d, e, \delta=0}^{q, r, s, 1} C(c, d, e, \delta) \zeta_{1}^{c \delta} \in \pi^{q p^{2} k+r p k+s k+\left(p^{2} k\right)^{\prime}} R .
$$

Since this last sum is equal to

$$
\left(\sum_{d, e=0}^{r, s}\left(\begin{array}{l}
r \\
d
\end{array}\right)\left(\begin{array}{l}
s \\
e
\end{array}\right)(-1)^{r-d}(-1)^{s-e}\right)\left(\sum_{c, \delta=0}^{q, 1} C(c, \delta) \zeta_{1}^{c \delta}\right),
$$

and the first factor is 1 is $r, s=0$ and 0 otherwise, it suffices to show that

$$
\sum_{c, \delta=0}^{q, 1} C(c, \delta) \zeta_{1}^{c \delta} \in \pi^{q p^{2} k+\left(p^{2} k\right)^{\prime}} R,
$$

for $q=0, \ldots, p-1$, which holds. Thus by Theorem 4.8, one has

$$
A^{*}=R\left[\frac{\gamma^{p^{2}}-1}{\pi^{k^{\prime}}}, \frac{a_{\zeta_{2}^{-1}} \gamma^{p}-1}{\pi^{(p k)^{\prime}}}, \frac{a_{\left(v \zeta_{3}\right)^{-1}} b_{\zeta_{2}^{-1}} \gamma-1}{\pi^{\left(p^{2} k\right)^{\prime}}}\right] .
$$

The realizable Hopf orders of Theorem 5.6 and Theorem 5.7 are distinct as the following example shows.

Example 5.8. Set $p=3, e^{\prime}=300, k=30, \operatorname{ord}(v-1)=40$. Then

$$
A=R\left[\frac{g^{9}-1}{\pi^{270}}, \frac{g^{3}-1}{\pi^{90}}, \frac{a_{v} g-1}{\pi^{30}}\right]
$$

is a monogenic $R$-Hopf order in $K C_{27}$ with realizable linear dual

$$
A^{*}=R\left[\frac{\gamma^{9}-1}{\pi^{270}}, \frac{a_{\zeta_{2}^{-1}} \gamma^{3}-1}{\pi^{210}}, \frac{a_{\left(v \zeta_{3}\right)^{-1}} b_{\zeta_{2}^{-1}} \gamma-1}{\pi^{30}}\right] .
$$

Note $\mathcal{L}\left(R\left[\frac{\gamma^{9}-1}{\pi^{270}}, \frac{{ }_{\zeta_{2}} \gamma^{3}-1}{\pi^{210}}\right]\right)=H(270,70)$. But $3(30) \not \leq 70$, so $A^{*}$ cannot be of the form of Theorem 5.6. 
Remark 5.9. Let $A_{0}=A\left(p^{2} k, k, v\right)$ and $A=H\left(p^{2} k, p k, k, 1, v, 1\right)$ be the Hopf orders of Theorem 5.7, and assume $p^{2} k<e^{\prime}$ and ord $\left(v^{p}-1\right)=b$ with $e^{\prime}>b>e^{\prime} / p$. We show that for suitable choice of $k, A^{*}$ is not an ILD order, not a formal group Hopf order, not the dual of a formal group Hopf order, and not a duality Hopf order. Thus $A^{*}$ is not any of the types constructed earlier in this paper.

To show that $A^{*}$ cannot be an ILD Hopf order, we first draw consequences from the fact that $A_{0}$ is Greither. Since $\operatorname{ord}\left(v^{p} \zeta_{1}-1\right)=b$, we have

$$
\begin{gathered}
b \geq e^{\prime}-p^{2} k+p k, \\
b \geq p\left(e^{\prime}-p^{2} k\right)+k .
\end{gathered}
$$

Since $b<e^{\prime}$, (6) implies

$$
p^{3} k-k \geq p e^{\prime}-e^{\prime}
$$

Hence

$$
p^{2} k \geq\left(\frac{p^{3}-p^{2}}{p^{3}-1}\right) e^{\prime}
$$

Now $A^{*}=A\left(k^{\prime},(p k)^{\prime},\left(p^{2} k\right)^{\prime}, \zeta_{2}^{-1},\left(v \zeta_{3}\right)^{-1}, \zeta_{2}^{-1}\right)$ is an extension of a rank $p$ Larson order by a Larson dual. Hence if $A^{*}$ is ILD, then $A^{*}$ is cohomological. For that to occur, we require conditions on the valuation parameters, namely

$$
p\left(e^{\prime}-p^{2} k\right) \leq e^{\prime}-p k,
$$

which implies

$$
p^{2} k \geq\left(\frac{p^{3}-p^{2}}{p^{3}-p}\right) e^{\prime}
$$

and

$$
k+p\left(e^{\prime}-p^{2} k\right) \leq e^{\prime} / p
$$

which implies

$$
p^{2} k \geq\left(\frac{p^{3}-p}{p^{3}-1}\right) e^{\prime}
$$

Thus, recalling (7), if

$$
\left(\frac{p^{3}-p^{2}}{p^{3}-1}\right) e^{\prime} \leq p^{2} k<\left(\frac{p^{3}-p^{2}}{p^{3}-p}\right) e^{\prime}<\left(\frac{p^{3}-p}{p^{3}-1}\right) e^{\prime},
$$

then $A^{*}$ is not cohomological. As an example, for $p=3, e^{\prime}=780, k=61$, then $(18 / 26) e^{\prime}=540<9 k=549<(18 / 24) e^{\prime}=585$.

Also, $A^{*}$ is not a formal group Hopf order. To be a formal group Hopf order, we would require that $(p k)^{\prime} \geq p\left(p^{2} k\right)^{\prime}$, hence

$$
p^{2} k \geq\left(\frac{p^{3}-p^{2}}{p^{3}-p}\right) e^{\prime},
$$

hence the last example fails this inequality and $A^{*}$ is not a formal group Hopf order.

Neither can $A^{*}$ be the dual of a formal group Hopf order as constructed in Theorem 4.1 or Theorem 4.6. For suppose $A$ is a formal group Hopf order, $A=H_{\Theta}$. Then $\Theta$ must have the form

$$
\Theta=\left(\begin{array}{ccc}
\pi^{p^{2} k} & 0 & 0 \\
0 & \pi^{p k} & 0 \\
c \pi^{s} & 0 & \pi^{k}
\end{array}\right) .
$$


Then $A$ is not a formal group Hopf order as in Theorem 4.1 because there we require that $\operatorname{ord}\left(\theta_{r, r}\right) \geq d \operatorname{ord}\left(\theta_{r+1, r+1}\right)$ with $d>p$. Moreover, $A$ is not of the form of Theorem 4.6 since there we require that $b$ and $d$ be units of $R$.

The construction of Theorem 4.6 remains valid in the case that $b=d=0$, however, and the resulting formal group Hopf orders $H_{\Theta}$ have matrices of the form $\Theta$ with $2 s \geq k>s$, and $c$ a unit. We show that there exists $A$ not of the form $H_{\Theta}$. If $A=H_{\Theta}$, then $\left\langle H_{\Theta}, A^{*}\right\rangle \subset R$, so in particular,

$$
\left\langle\frac{g-1}{\pi^{k}}+\frac{-c \pi^{s}\left(g^{p^{2}}-1\right)}{\pi^{p^{2} k+k}}, \frac{a_{\left(v \zeta_{3}\right)^{-1}} b_{\zeta_{2}^{-1}} \gamma-1}{\pi^{\left(p^{2} k\right)^{\prime}}}\right\rangle \in R .
$$

But the expression in angle brackets is

$$
\frac{v^{-1}-1}{\pi^{\left(p^{2} k\right)^{\prime}+k}}+\frac{-c \pi^{s-p^{2} k}}{\pi^{\left(p^{2} k\right)^{\prime}+k}}\left(\zeta_{1}-1\right)
$$

so we require that

$$
v^{-1} \equiv 1+c \pi^{s-p^{2} k}\left(\zeta_{1}-1\right) \quad\left(\bmod \pi^{\left(p^{2} k\right)^{\prime}+k} R\right) .
$$

Since $\left(p^{2} k\right)^{\prime}+s<\left(p^{2} k\right)^{\prime}+k$, ord $(1-v)=\left(p^{2} k\right)^{\prime}+s$, hence we require that $\operatorname{ord}(1-v) \geq\left(p^{2} k\right)^{\prime}+(k / 2)$. Now since $e^{\prime} / p>\operatorname{ord}(1-v)$, any $k$ for which $\left(p^{2} k\right)^{\prime}+$ $(k / 2)>e^{\prime} / p$ yields $A$ is not a formal group. In fact, the example given earlier provides such a $k$.

Finally, since $\operatorname{ord}\left(1-v^{p}\right)>e^{\prime} / p, A$ is monogenic, hence $A^{*}$ is realizable. Thus neither $A^{*}$ nor $A$ can be duality by Theorem 5.3.

\section{REFERENCES}

[By93] N. Byott, Cleft extensions of Hopf algebras, Proc. London Math. Soc. 67 (1993), 227307. MR $1226603(94 \mathrm{~m}: 16044)$

[By02a] N. Byott, Integral Hopf-Galois structures in degree $p^{2}$ extensions of $p$-adic fields, $J$. Alg., 248 (2002), 334-365. MR 1879021 (2002j:11142)

[By04] N. Byott, Monogenic Hopf orders and associated orders of valuation rings, J. Algebra 275 (2004), 575-599. MR2052627

[C96] L. N. Childs, Hopf Galois structures on degree $p^{2}$ cyclic extensions of local fields, New York J. of Math., 2 (1996), 86-102. MR1420597(97j:11058)

[C98] L. N. Childs, Introduction to polynomial formal groups and Hopf algebras, Memoirs Am. Math. Soc., 136, no. 651 (1998), 1-10. MR1629460 (2000c:14067)

[C00] L. N. Childs, Taming Wild Extensions: Hopf Algebras and Local Galois Module Theory, American Mathematical Society, Mathematical Surveys and Monographs 80, 2000. MR 1767499 (2001e:11116)

[CMS98] L. N. Childs, D.J. Moss, J. Sauerberg, Dimension one polynomial formal groups, Memoirs Am. Math. Soc., 136, no. 651 (1998), 11-19. MR1629460 (2000c:14067)

[CMSZ98] L. N. Childs, D.J. Moss, J. Sauerberg, K. Zimmermann, Dimension two polynomial formal groups, Memoirs Am. Math. Soc., 136, no. 651 (1998), 21-54. MR1629460 (2000c:14067)

[CS98] L.N. Childs, J. Sauerberg, Degree two formal groups and Hopf algebras, Memoirs Amer. Math. Soc., 136, no. 651 (1998), 55-89. MR1629460 (2000c:14067)

[CU03] L. N. Childs, R.G. Underwood, Cyclic Hopf orders defined by isogenies of formal groups, Amer. J. Math., 125 (2003), 1295-1334. MR2018662(2004i:11138)

[CZ94] L. N. Childs, K. Zimmermann, Congruence-torsion subgroups of dimension one formal groups, J. Alg., 170 (1994), 929-955. MR1305271 (95k:14067)

[Gr92] C. Greither, Extensions of finite group schemes, and Hopf Galois theory over a complete discrete valuation ring, Math. Z., 210 (1992), 37-67. MR1161169 (93f:14024) 
[GC98] C. Greither, L. N. Childs, p-elementary group schemes-constructions, and Raynaud's theory, Memoirs Am. Math. Soc., 136, no. 651 (1998), 91-117. MR1629460 (2000c:14067)

[La76] R. G. Larson, Hopf algebra orders determined by group valuations, J. Alg., 38 (1976), 414-452. MR 0404413 (53:8215)

[Lu79] J. Lubin, Canonical subgroups of formal groups, Trans. Am. Math. Soc., 251 (1979), 103-127. MR0531971 (80j:14039)

[Sm97] H. Smith, Constructing Hopf orders in elementary abelian group rings, doctoral dissertation, SUNY Albany (1997).

[SS94] T. Sekiguchi, N. Suwa, Theories de Kummer-Artin-Schreier-Witt, Comptes Rendus de l'Acad. des Sci., 319, ser. I (1994), 105-110. MR.1288386

[TO70] F. Tate, J. Oort, Group schemes of prime order, Ann. Sci. Ec. Norm. Sup., 3 (1970), 1-21. MR $0265368(42: 278)$

[Un94] R. Underwood, $R$-Hopf algebra orders in $K C_{p^{2}}, J$. Alg., 169 (1994), 418-440. MR $1297158(95 \mathrm{k}: 16055)$

[Un96] R. Underwood, The valuative condition and $R$-Hopf algebra orders in $K C_{p^{3}}, A m e r . J$. Math., 118 (1996), 401-743. MR.1400057 (97e:11150)

[Un98] R. Underwood, The structure and realizability of $R$-Hopf orders in $K_{p^{3}}$, Comm. Alg., 26(11) (1998), 3447-3462. MR.1647146 (2000a:16073)

[Un99] R. Underwood, Isogenies of polynomial formal groups, J. Alg., 212 (1999), 428-459. MR 1676848 (99m:14084)

[Un03] R. Underwood, Galois module theory over a discrete valuation ring, in "Recent Research on Pure and Applied Algebra", Nova Science Publishers, New York, 2003. MR2030462

Department of Mathematics, Auburn University Montgomery, Montgomery, Alabama 36124

Department of Mathematics and Statistics, SUny at Albany, Albany, New York 12222 